\title{
Induction of cerebral hyperexcitability by peripheral viral challenge: Role of CXCL10 chemokine
}

\author{
Tiffany J. Petrisko \\ West Virginia University, tjpetrisko@mix.wvu.edu
}

Follow this and additional works at: https://researchrepository.wvu.edu/etd

Part of the Molecular and Cellular Neuroscience Commons, and the Other Immunology and Infectious Disease Commons

\section{Recommended Citation}

Petrisko, Tiffany J., "Induction of cerebral hyperexcitability by peripheral viral challenge: Role of CXCL10 chemokine" (2019). Graduate Theses, Dissertations, and Problem Reports. 7452.

https://researchrepository.wvu.edu/etd/7452

This Dissertation is protected by copyright and/or related rights. It has been brought to you by the The Research Repository @ WVU with permission from the rights-holder(s). You are free to use this Dissertation in any way that is permitted by the copyright and related rights legislation that applies to your use. For other uses you must obtain permission from the rights-holder(s) directly, unless additional rights are indicated by a Creative Commons license in the record and/ or on the work itself. This Dissertation has been accepted for inclusion in WVU Graduate Theses, Dissertations, and Problem Reports collection by an authorized administrator of The Research Repository @ WVU.

For more information, please contact researchrepository@mail.wvu.edu. 
Induction of cerebral hyperexcitability by peripheral viral challenge: Role of CXCL10 chemokine

\title{
Tiffany J. Petrisko
}

\author{
Dissertation \\ submitted to the School of Medicine \\ at West Virginia University
}

in partial fulfillment of the requirements for the degree of

\author{
Doctor of Philosophy \\ in \\ Neuroscience
Gregory Konat, Ph.D., Chair
Candice Brown, Ph.D.
Jason Huber, Ph.D.
Rosana Schafer, Ph.D. James Simpkins, Ph.D. \\ Departments of Biochemistry and Neuroscience
}

Morgantown, West Virginia

2019

Keywords: antiviral acute phase response, CXCL10/CXCR3 axis, neuronal hyperexcitability, microglia

\section{Copyright 2019 Tiffany J. Petrisko}




\begin{abstract}
Induction of Cerebral Hyperexcitability by Peripheral Viral Infection: Role of CXCL10 Chemokine
\end{abstract}

Tiffany J. Petrisko

Peripheral viral infections are potent comorbid factors that exacerbate neurodegeneration. Although the underlying mechanisms have not been defined, neuronal hyperexcitability has been established as an underlying feature. Our lab has developed a preclinical model in which a viral mimetic, polyinosinic-polycytidylic acid (PIC) is injected intraperitoneally to induce an antiviral acute phase response (APR). APR in turn elicits robust neuronal hyperexcitability. The present study was undertaken to characterize molecular mechanisms that mediate the development of hyperexcitability in response to PIC challenge. The analysis of brain tissue after PIC challenge revealed a robust elevation of CXCL10 chemokine, indicating its putative role in the process. We have further shown that CXCL10 is overwhelmingly produced by neurons. Neurons also express the cognate receptor, CXCR3, indicating that the CXCL10/CXCR3 axis acts in an autocrine/paracrine manner. Intracerebroventricular injection of CXCR3 inhibitor, AMG-487, abrogates PIC challenge-induced development of hyperexcitability, indicating that the CXCL10/CXCR3 axis drives this process. Moreover, CXCR3 signaling activates microglia. Because microglia are potent modulators of synaptic activity, they likely mediate the development of hyperexcitability. Accordingly, we posit that CXCL10/CXCR3 axis induces the generation of neuronal factors that activate microglia. Activated microglia in turn, impede the activity of inhibitory synapses. This de-inhibition results in the hyperexcitability of neuronal networks. Our results provide a springboard for the full elucidation of the underlying mechanisms that would facilitate the development of novel therapeutic modalities to combat the co-morbidity of peripheral viral infections in neurodegenerative diseases. 


\section{Acknowledgements}

Firstly, I wish to express my sincerest gratitude to my advisor, Dr. Gregory Konat, for his constant support, guidance, and patience throughout my Ph.D. training. I cannot adequately express how much your mentorship and unwavering belief in me have helped me grow, both as a scientist and as a person these past few years. Thank you.

I would also like to thank my committee members, Dr. Candice Brown, Dr. Jason Huber, Dr. Rosana Schafer, and Dr. James (Jim) Simpkins for their insight and guidance throughout this work.

In addition, I would like to thank the recent undergraduate researchers who have passed through the Konat lab for their help in data collection, as well as all the previous members of the Konat lab, as well as our collaborators.

To the Microscopy Imaging Facility, in particular Drs. Karen Martin and Amanda (Mandy) Ammer for the invaluable time, patience, and support throughout all my microscopy imaging and data analysis. Without your assistance my dissertation would not have been possible.

To Dr. Randy Nelson and the Neuroscience Department, for giving me the opportunity to give back to our Ph.D. program as well as their constant support. To Erica Stewart, Morgan Prunty, and Cassie George for always being there to help out, solve problems, or just talk. Our Department and Program would be lost without you all! To Dr. Liz Engler-Chiurazzi, for being a wonderful role-model in science, outreach, and life. And to all my fellow Neuroscience graduate students, especially those who contributed to our outreach events. You all have helped me plow through the rough times and most importantly enjoy the good ones.

And lastly, I would like to thank all my friends and family who have made this journey with me. To my parents, your constant love, support, humor, and perseverance always gives me the strength to continue onward. To David, for always being there for me and knowing you're always in my corner. To my grandparents and all my aunts, uncles, cousins, and so on. Being so close to everyone these past few years has been wonderful. Thank you for always cheering me on. 


\section{Table of Contents}

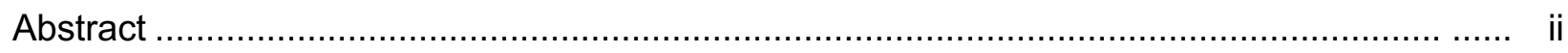

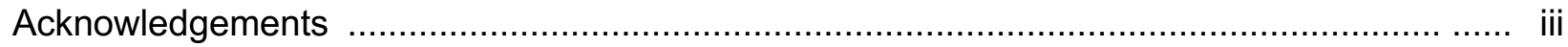

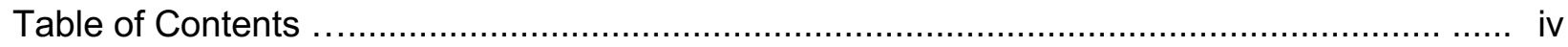

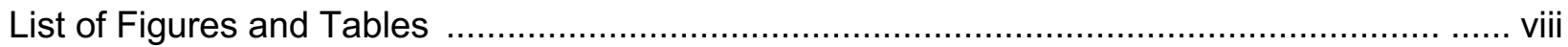

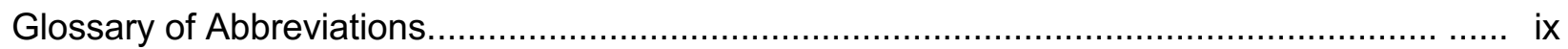

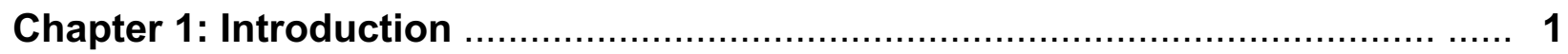

1.1 Immune-to-Brain Communication . .................................................................. 1

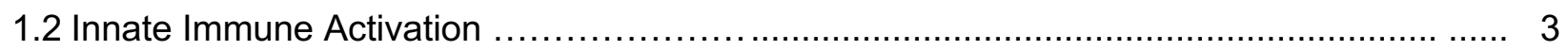

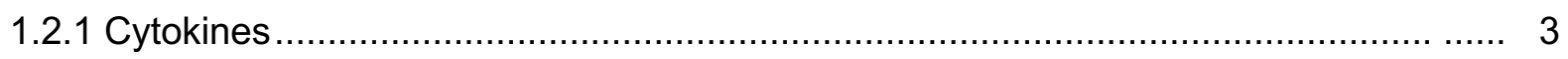

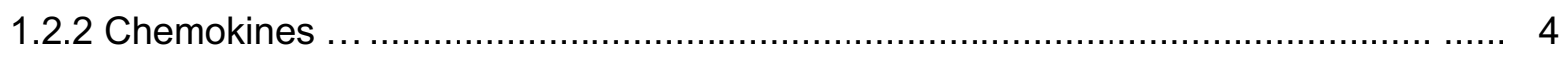

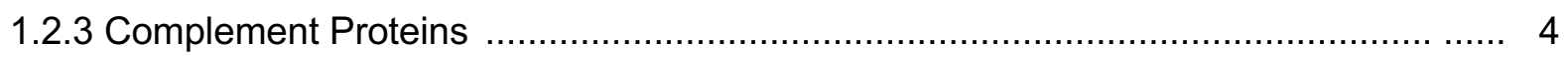

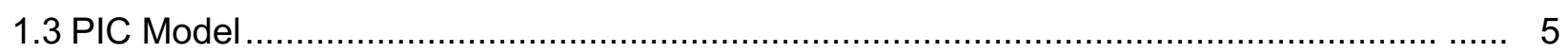

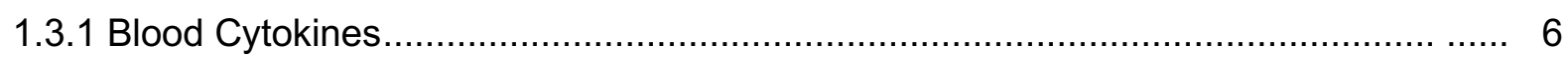

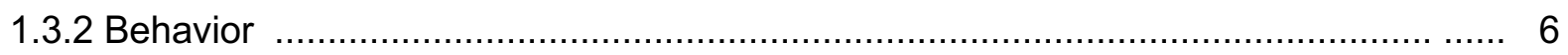

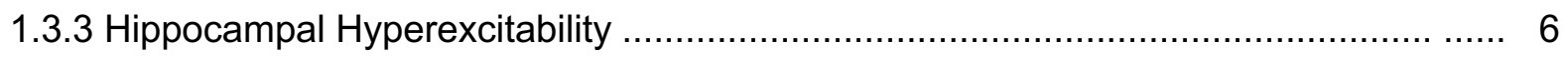

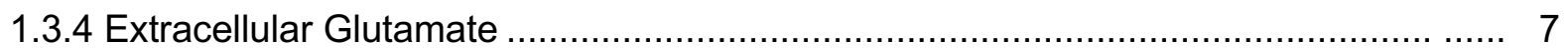

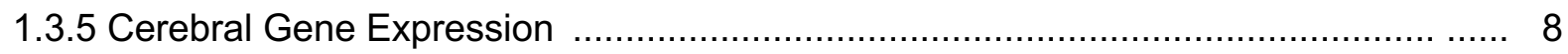

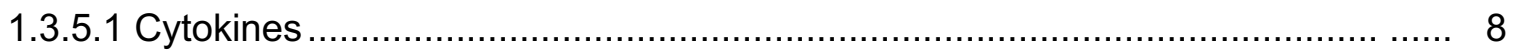

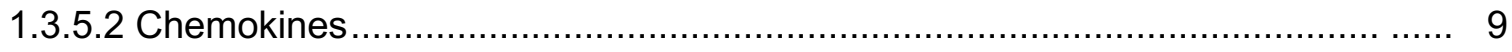

1.3.5.3 Complement Proteins ..................................................................... 9

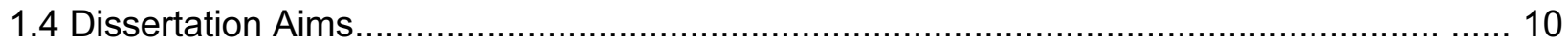

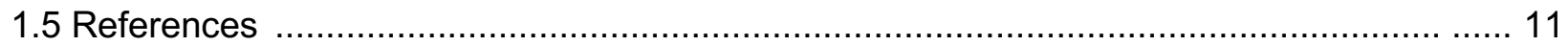

Chapter 2: Peripheral viral challenge triggers hippocampal production of inflammatory proteins (Petrisko and Konat, 2017, Metabolic Brain Disease) .......... 17

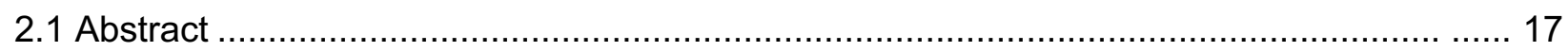

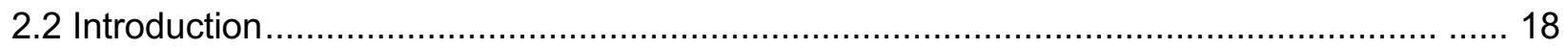

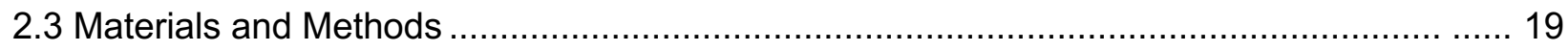

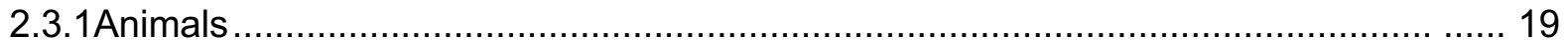

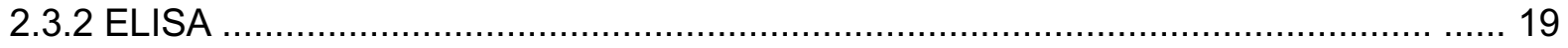

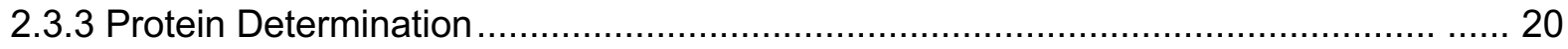




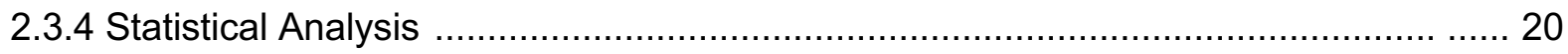

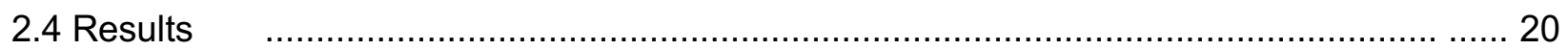

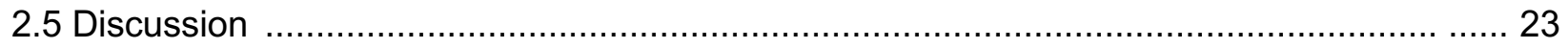

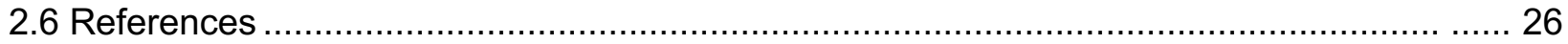

Chapter 3: Neuronal CXCL10/CXCR3 axis mediates the induction of cerebral hyperexcitability by peripheral viral challenge (Petrisko et al, under review, Frontiers

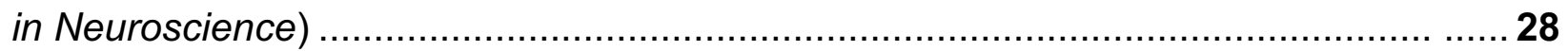

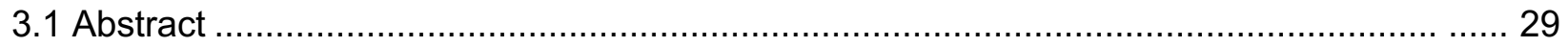

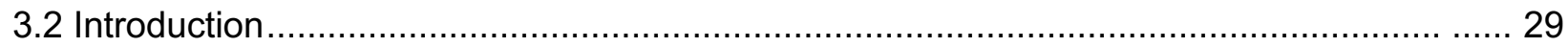

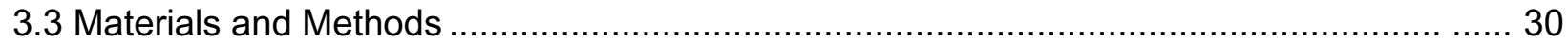

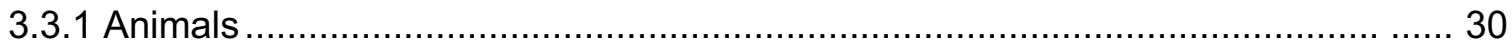

3.3.2 Drug Administration ............................................................................. 30

3.3.3 Induction of Seizures ............................................................................. 30

3.3.4 Extracellular Field Potential Recording ..................................................... 31

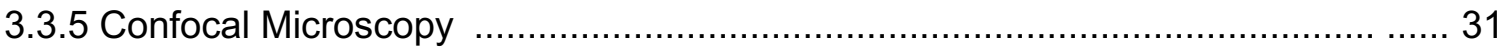

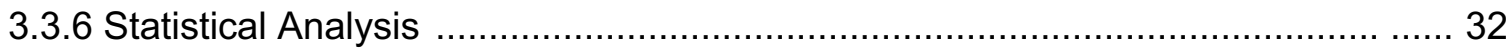

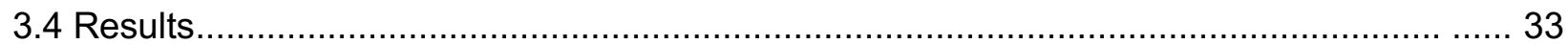

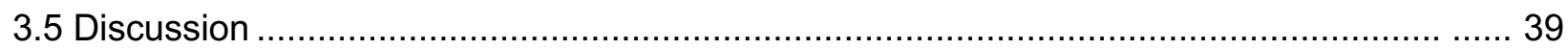

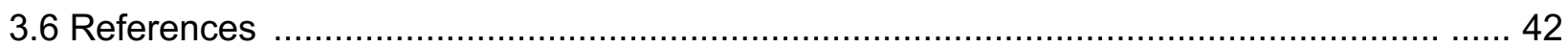

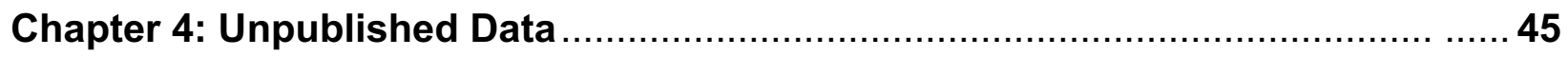

4.1 Spatiotemporal CXCL10 expression induced by PIC challenge .................................... 45

4.2 Neuronal c-fos expression as an index of hyperexcitability ............................................ 49

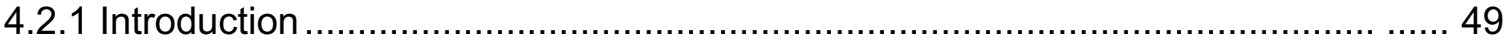

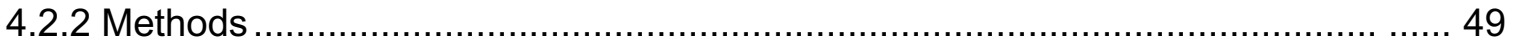

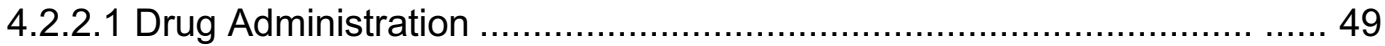

4.2.2.2 Microscopy ……............................................................................ 49

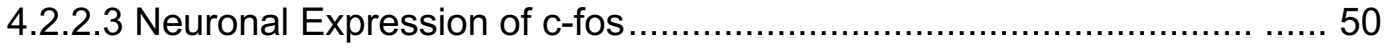

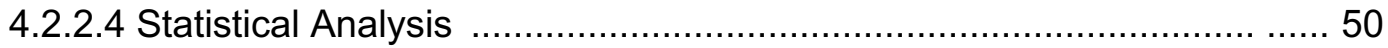

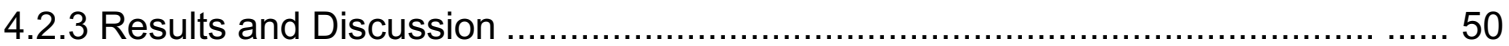

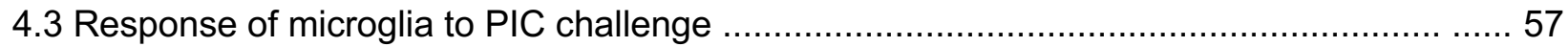

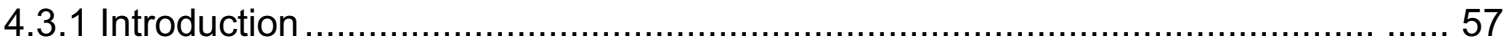

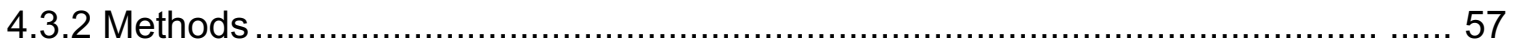




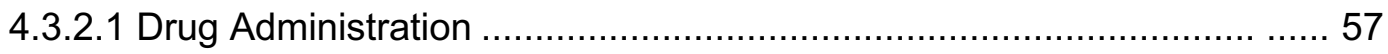

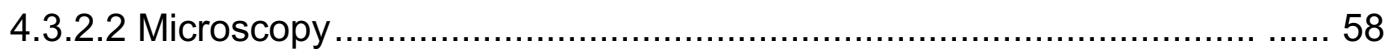

4.3.2.3 Neuron-Microglia Surface Area Contact .......................................... 58

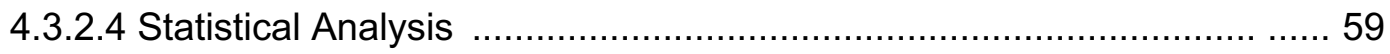

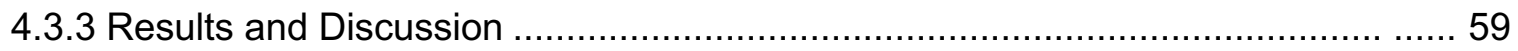

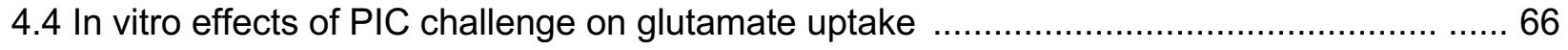

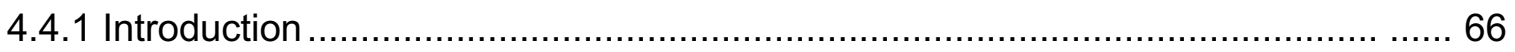

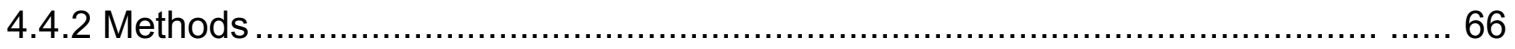

4.4.2.1 Preparation of Hippocampal Slices................................................... 66

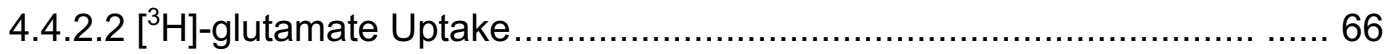

4.4.2.3 Protein Determination ................................................................. 67

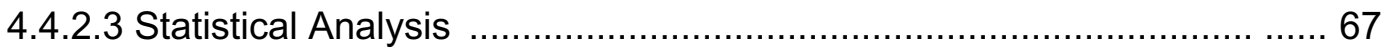

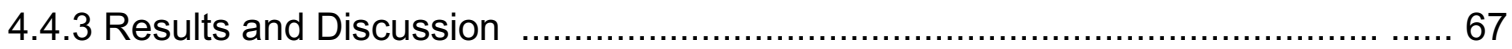

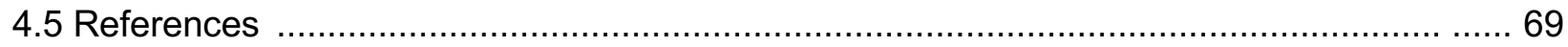

Chapter 5: Peripheral viral challenge exacerbates experimental autoimmune encephalomyelitis (Petrisko and Konat, 2019, Metabolic Brain Disease) .................. 72

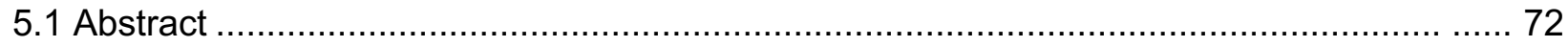

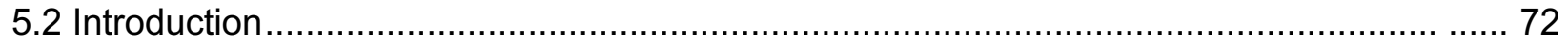

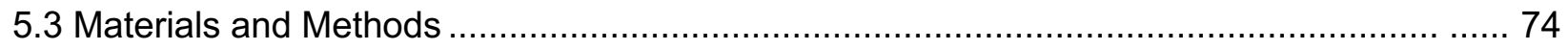

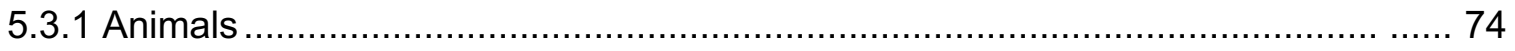

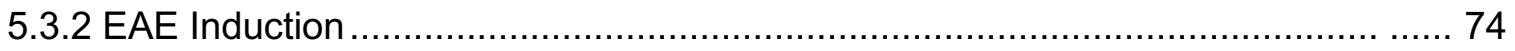

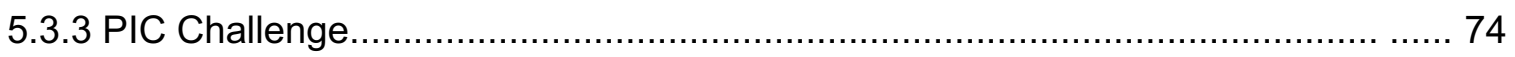

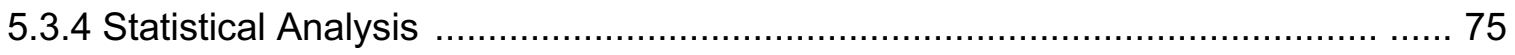

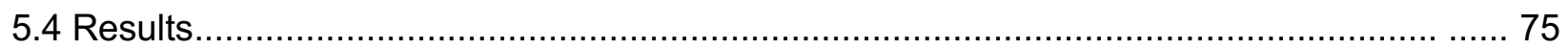

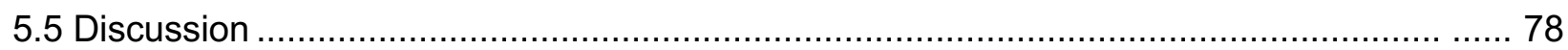

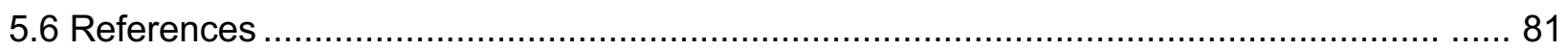

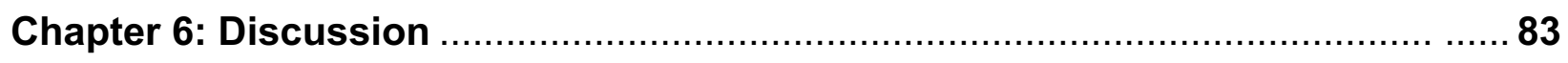

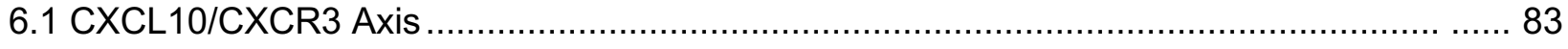

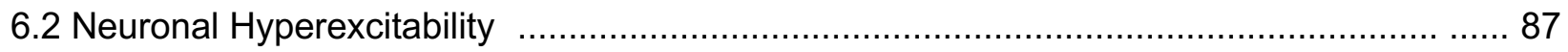

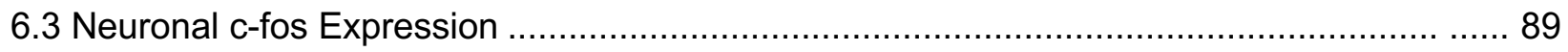

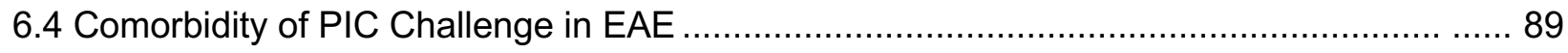

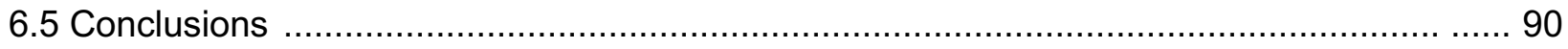




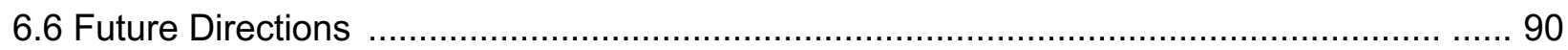

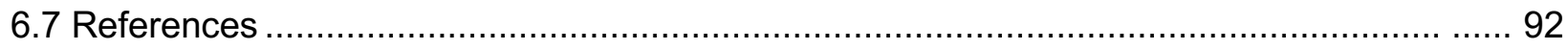




\section{List of Figures and Tables}

2.6.1 Production of chemokines induced by peripheral PIC challenge ............................... 21

2.6.2 Production of IL-1 $\beta, \mathrm{IL}-6$, and TNF $\alpha$ induced by peripheral PIC challenge ................... 22

2.6.3 Hippocampal production of the complement proteins induced by

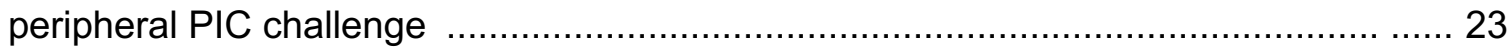

3.6.1 Cellular origin of cerebral CXCL10 production following PIC challenge........................ 34

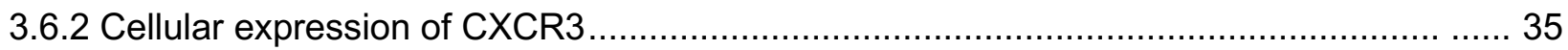

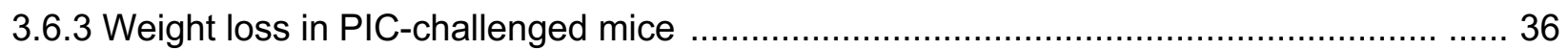

3.6.4 Synaptic transmission in hippocampal slices ................................................... 37

3.6.5 Synaptic plasticity in hippocampal slices ............................................................ 38

3.6.6 CXCR3 inhibition attenuates seizure hypersensitivity induced by PIC challenge ........... 39

4.1.1.1 Time course of neuronal CXCL10 expression in different brain regions following PIC

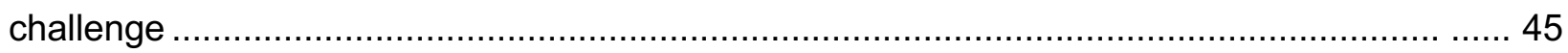

4.1.1.2 Time course of neuronal CXCL10 expression in different brain regions following PIC

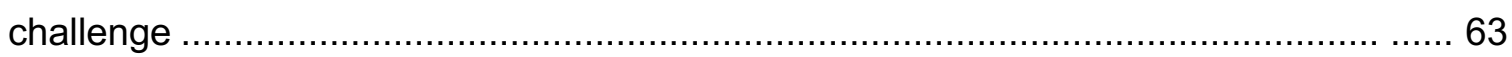

4.1.3.2 Cerebral generation of CXCL10 induced by PIC challenge ................................ 48

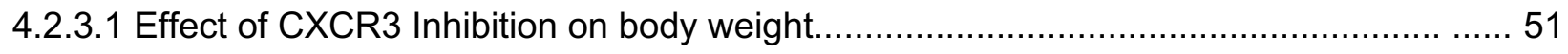

4.2.3.2 Effect of CXCR3 Inhibition on rearing behavior.............................................. 52

4.2.3.3 Time course of neuronal c-fos expression following PIC challenge .......................... 53

4.2.3.4 Temporal expression of neuronal c-fos instigated by PIC challenge. ........................ 54

4.2.3.5 Effect of CXCR3 inhibition on PIC challenge - induced neuronal c-fos expression ...... 55

4.2.3.6 Effect of CXCR3 inhibition on C-fos expression................................................... 56

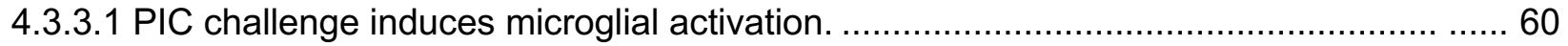

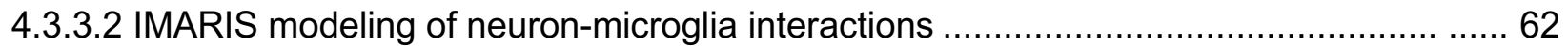

4.3.3.3 Interaction of microglia with cortical neurons following PIC challenge...................... 64

4.3.3.4 CXCR3 Inhibition attenuates microglia activation induced by PIC .......................... 65

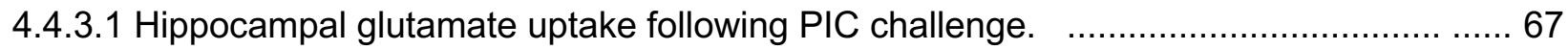

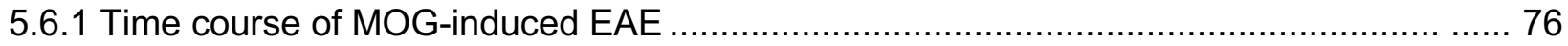

5.6.2 Comorbidity of PIC-induced APR during chronic phase of EAE ............................. 77

6.2.1 Signaling pathways activated following CXCR3 activation.................................... 84

6.2.2 Proposed mechanism of CXCL10/CXCR3-mediated induction of hyperexcitability ........ 88 


\section{Glossary of Abbreviations}

$\begin{array}{ll}{ }^{3} \mathrm{H} & \text { tritium } \\ \text { ACSF } & \text { artificial cerebral spinal fluid } \\ \text { AD } & \text { Alzheimer's disease } \\ \text { AKT } & \text { Protein Kinase B } \\ \text { AMG } & \text { AMG-487 } \\ \text { AMPA } & \alpha \text {-amino-3-hydroxy-5-methyl-4-isoxazolepropionic acid } \\ \text { ANOVA } & \text { analysis of variance } \\ \text { APR } & \text { acute phase response } \\ \text { BBB } & \text { blood brain barrier } \\ \text { BDNF } & \text { bone derived neurotrophic factor } \\ \text { C(2,3,..) } & \text { complement component } \\ \text { CA1 } & \text { cornu ammonis 1 } \\ \text { CA3 } & \text { cornu ammonis 3 } \\ \text { CCL } & \text { C-C motif chemokine ligand } \\ \text { CCR } & \text { C-C motif chemokine receptor } \\ \text { CD } & \text { cluster of differentiation } \\ \text { CFA } & \text { complete Freund's adjuvant } \\ \text { CFB } & \text { complement factor b } \\ \text { CNS } & \text { central nervous system } \\ \text { CPM } & \text { counts per minute } \\ \text { CSF } & \text { cerebral spinal fluid } \\ \text { CSS } & \text { cumulative seizure score } \\ \text { CTX } & \text { cortex } \\ \text { CVO } & \text { circumventricular organ } \\ \text { CXCL } & \text { C-X-C motif chemokine ligand } \\ \text { CXCR } & \text { damage associated molecular pattern } \\ \text { DAMP } & \text { differentially expressed gene } \\ \text { DEG } & \text { dentate gyrus } \\ \text { DG } & \text { excitatory amino acid transporter } \\ \text { DMSO } & \text { EAmental autoimmune encephalomyelitis } \\ \text { EAAT } & \text { EAE }\end{array}$




\begin{tabular}{|c|c|}
\hline ELISA & enzyme-linked immunosorbent assay \\
\hline ERK & extracellular signal-regulated kinases \\
\hline fEPSP & field excitatory post synaptic potential \\
\hline GABA & gamma-aminobutyric acid \\
\hline $\mathrm{GABA}_{\mathrm{A}} \mathrm{R} \alpha 2$ & $\mathrm{GABA}_{\mathrm{A}}$ receptor subunit alpha 2 \\
\hline $\mathrm{GABA}_{B} \mathrm{R} 1$ & $\mathrm{GABA}_{\mathrm{B}}$ receptor subunit 1 \\
\hline GABRE & GABA receptor subunit epsilon \\
\hline GAD & glutamate decarboxylase \\
\hline i.c.v. & intracerebroventricular \\
\hline i.p. & intraperitoneal \\
\hline IEG & immediate-early gene \\
\hline IFN & interferon \\
\hline IL & interleukin \\
\hline iNOS & inducible nitric oxide synthase \\
\hline IP-10 & CXCL10 \\
\hline IP3 & inositol triphosphate \\
\hline ITAC & CXCL11 \\
\hline KA & kainic acid \\
\hline LPS & lipopolysaccharide \\
\hline LTD & long-term depression \\
\hline LTP & long-term potentiation \\
\hline MAC & membrane attack complex \\
\hline MDA & melanoma differentiation-associated protein \\
\hline mGLUR2/3 & metabotropic glutamate receptor $2 / 3$ \\
\hline MIG & CXCL9 \\
\hline MOG & myelin oligodendrocyte glycoprotein \\
\hline mRNA & messenger RNA \\
\hline MS & multiple sclerosis \\
\hline NMDA & N-methyl-D-aspartate \\
\hline NMDAR1 & NMDA receptor type 1 \\
\hline PAMP & pathogen associated molecular pattern \\
\hline PD & Parkinson's disease \\
\hline PIC & polyinosinic:polycytidylic acid \\
\hline PKB & protein kinase $B$ \\
\hline
\end{tabular}




$\begin{array}{ll}\text { PKC } & \text { protein kinase C } \\ \text { PKR } & \text { protein kinase R } \\ \text { PPF } & \text { paired pulse facilitation } \\ \text { PRR } & \text { pattern recognition receptor } \\ \text { PTx } & \text { pertussis toxin } \\ \text { qRT-PCR } & \text { quantitative real-time polymerase chain reaction } \\ \text { RIG } & \text { retinoic acid inducible gene } \\ \text { RMANOVA } & \text { repeated measures analysis of variance } \\ \text { RNA } & \text { ribonucleic acid } \\ \text { S.C. } & \text { subcutaneous } \\ \text { SAL } & \text { saline } \\ \text { SDS } & \text { sodium dodecyl sulfate } \\ \text { SEM } & \text { standard error of the mean } \\ \text { SXC } & \text { system xc cystine/glutamate antiporter } \\ \text { TBOA } & \text { dl-threo-beta-benzyloxyaspartate } \\ \text { Th(1,2..) } & \text { T-helper } \\ \text { TIR } & \text { Toll-interleukin-1 (IL-1) receptor } \\ \text { TLR } & \text { toll like receptor } \\ \text { TNF } & \text { tumor necrosis factor } \\ \text { TRIF } & \text { TIR domain-containing adaptor protein inducing interferon- } \beta \\ \text { WNV } & \text { West Nile Virus } \\ & \end{array}$




\section{CHAPTER 1: INTRODUCTION}

\subsection{IMMUNE-TO-BRAIN COMMUNICATION}

The existence of a bi-directional communication between the peripheral immune system and the brain has been recognized for many years. One of the most well-known examples of immuneto-brain communication can be observed following activation of the innate immune system by a pathogen. Recognition of the pathogen initiates systemic production of pro-inflammatory factors, the process referred to as the acute phase response (APR). Blood-borne APR factors, in turn, induces a "mirror" inflammatory response in the central nervous system (CNS) (McCusker and Kelley, 2013). This cerebral response then elicits alterations in neurochemical and neuroendocrine signaling, resulting in the induction of a group of symptoms collectively known as "sickness behavior". Sickness behavior represents an adaptive behavioral response consisting of fever, depression, anorexia, malaise, anxiety, cognitive dysfunction, and fatigue consistently observed across animal species (Hart, 1988; Quan and Banks, 2007; Dantzer, 2009).

The induction of a cerebral response by peripheral APR can occur by different mechanisms. Blood-borne inflammatory mediators can be directly transported from the circulation by the endothelial cells that form the blood brain barrier (BBB). Inflammatory mediators can also be transduced by the endothelial cells releasing secondary mediators into the brain parenchyma. Blood-borne mediators can also enter the brain through circumventricular organs (CVOs). In contrast to the most areas of the brain containing continuous capillaries, CVOs contain fenestrated capillaries, and thus provide no BBB (Ganong, 2000). Once inflammatory mediators enter the brain, they are able to activate the innate immune cells of the brain, mainly astrocytes and microglia, which in response generate additional inflammatory mediators. In addition, neurons express a multitude of cytokine receptors, and therefore can also generate additional mediators. Overall, cerebral cells are exposed to a combination of peripherally and centrally generated inflammatory mediators which modulate neurotransmitter and neuroendocrine systems, and ultimately alter brain function resulting in the sickness behavior (Konat, 2016). Finally, peripherally-generated cytokines may activate afferents of the vagal nerve which conveys the stimulus to multiple nuclei located in the medulla oblongata, and induce several symptoms of sickness behavior, including fever, taste aversion, anorexia and hyperalgesia (Fleshner et al., 1995; Watkins et al., 1995). 
Despite resulting in uncomfortable side effects, sickness behavior is a survival adaption that alters organisms' priorities to eliminate pathogens by conserving metabolic resources and preventing the spread of the pathogen within the population (Adelman and Martin, 2009; Shakhar and Shakhar, 2015). Additionally, the cerebral inflammatory response is neuroprotective, likely by priming the brain against plausible future insults. Indeed, multiple studies have shown that systemic pretreatments of animals with bacterial endotoxin, lipopolysaccharide (LPS), or viral mimetic polyinosinic: polycytidylic acid (PIC) reduce stroke volume, white matter damage following traumatic brain injury, and nitric oxide toxicity (Huang et al., 2008; Packard et al., 2012; Turner et al., 2017; Lv et al., 2018; Yu et al., 2018).

While APR is neuroprotective to the healthy brain, it is detrimental to the pathological brain. Peripheral infections have long been known to exacerbate symptoms in patients suffering from neurological disorders. Examples of this exacerbation can be observed in patients with seizure syndromes, such as epilepsy, in which both the incidence and severity of seizure, is increased (Tellez-Zenteno et al., 2005; Vezzani and Granata, 2005; Verrotti et al., 2009). In patients with multiple sclerosis (MS), bacterial urinary tract infections as well as upper respiratory infections, such as influenza, have been correlated with an increased incidence of relapses (Andersen et al., 1993; Panitch, 1994; Edwards et al., 1998; Buljevac et al., 2002; Libbey and Fujinami, 2010). Viral infections have been demonstrated to hasten cognitive decline and induce delirium in Alzheimer's disease (AD) patients (Murray et al., 1993; Nee and Lippa, 1999; Holmes et al., 2003; Holmes, 2013). In Parkinson's disease (PD), respiratory infections have been found to be the most frequent cause of increased motor impairments (George et al., 1997; Zheng et al., 2012; Brugger et al., 2015). Additionally, post-stroke systemic infections increase disability and mortality of the victims (Westendorp et al., 2011; Learoyd et al., 2017).

Although the correlation between peripheral infections and neurological exacerbations has been well documented, the mechanisms underlying induction of these exacerbations remain poorly understood. However, there is a compelling body of evidence that the cerebral response is mediated by the initial response of the innate immune system to infection, i.e., the APR (Dantzer, 2006; Cunningham et al., 2007; McCusker and Kelley, 2013; Mardiguian et al., 2017). 


\subsection{INNATE IMMUNE ACTIVATION}

The innate immune system is the body's first line of defense against infectious and/or other insults. The innate immune system relies on the activation of pattern recognition receptors (PRRs) expressed predominantly on innate immune cells, including dendritic cells, leukocytes and tissue macrophages, as well as many types of somatic cells (Medzhitov, 2001). PRRs have evolved to recognize common patterns of the molecular structures that are fond in pathogens, called pathogen-associated molecular patterns (PAMPs), as well as endogenous molecules released from damaged and/or dying cells, called damage associated molecular patterns (DAMPs) (Takeuchi and Akira, 2010). An important family of PRRs are toll-like receptors (TLRs), which are found on the plasma membrane, endosomes, and lysosomes, permitting them to recognize extra-cellular and intra-cellular PAMPs and DAMPs. Ligation of a specific TLR with its corresponding PAMP/DAMP results in the activation of downstream signaling pathways, and subsequently, the production and secretion of cytokines, chemokines and other inflammatory mediators at the site of infection (Baumann and Gauldie, 1994; Kushner and Rzewnicki, 1994; Cray et al., 2009; Jain et al., 2011). In addition to their local action, the inflammatory mediators quickly enter the blood stream to induce the APR. Of note, APR entails the activation of other tissues and organs, notably, the liver that propagate the inflammatory reaction by generating additional factors. The resultant cocktail of circulating inflammatory factors includes chiefly cytokines, chemokines and complement proteins.

\subsubsection{CYTOKINES}

Cytokines are a broad category of small peptides, typically less than $80 \mathrm{kDa}$ in size, which are secreted by a variety of cell types to regulate a range of biological functions, including the innate immune system, inflammation, and hematopoiesis. These extracellular signaling proteins can function in autocrine, paracrine, and endocrine manners to exert effects on both nearby cells and long-range targets. Cytokines can be further categorized based on the secreting cell type and function (Zhang and An, 2007; Holdsworth and Gan, 2015). For example, lymphokines and monokines are secreted by lymphocytes and monocytes, respectively. Interleukins are cytokines that are secreted by leukocytes and act upon other leukocytes. Interferons are antiviral cytokines that block the replication of viruses within infected cells. Cytokines can exert pro-inflammatory or anti-inflammatory functions, depending on their concentration, the activation signal, and the target cell (Cavaillon, 2001). Due to the diverse nature of cytokines, their receptors vary greatly in both structure and mechanisms of signal transduction (Wang et al., 2009). 


\subsubsection{ChemoKines}

Chemokines are a subclass of cytokines which are classically thought to function as chemoattractant signals that recruit immune cells to the site of infection (Turner et al., 2014). They are small, 8-10 kDa proteins characterized by a conserved amino acid structure. Most chemokines contain four cysteines that interact with one another through intramolecular disulfide bonds. Based on the structure and the spacing of their first cysteine residues, chemokines can be divided into four groups (Asensio and Campbell, 1999; Reaux-Le Goazigo et al., 2013). C chemokines contain only two cysteines, one near its $\mathrm{N}$ terminus and another located downstream. CC chemokines contain two adjacent cysteines near their amino terminus while CXC chemokines contain two $\mathrm{N}$-terminal cysteines separated by a single amino acid. Finally, $\mathrm{CX}_{3} \mathrm{C}$ chemokines have three amino acids between their two cysteine residues.

Chemokines primarily signal through 7-transmembrane G-protein coupled receptors (GPCRs). Receptor ligation results in homo or heterodimerization (Colvin et al., 2004) resulting in the dissociation of $\mathrm{G}$ protein subunits. This in turn, increases intracellular calcium, and activates downstream signaling pathways (Bajova et al., 2008). These pathways include the extracellular signal-regulated kinases (Ras/ERK), protein kinase $B(P I 3 K / A K T)$, and protein kinase $C(P K C)$ signaling pathways. Their activation induces cytoskeleton rearrangements, cell proliferation, migration, and adhesion of immune cells (Curnock et al., 2002; Legler and Thelen, 2018).

\subsubsection{COMPLEMENT PROTEINS}

Complement proteins are distinct plasma proteins that aid in the clearance of invading microbes and infected/damaged host cells. The complement proteins react with one another to generate activated complement proteins that bind covalently to pathogens and tag them for phagocytosis. The activation process also generates small fragments called anaphylatoxins that act as chemoattractants and propagate inflammatory responses. Finally, the terminal complement components assemble into the membrane attack complex (MAC), which forms transmembrane pores on the invading microbes or infected cells resulting in cell lysis and death. 


\subsection{PIC MODEL}

To model exacerbation of neurological disorders by peripheral viral infections, the Konat lab established a periclinal model utilizing intraperitoneal (i.p.) injection of the viral mimetic, PIC. PIC is a synthetic double stranded RNA (dsRNA), a PAMP of viral infections, as most viruses either consist of dsRNA or generate dsRNA during their replication cycles (Jacobs and Langland, 1996; Weber et al., 2006). Multiple PRRs are present on mammalian cells that detect both intracellular and extracellular dsRNA, including the endosomal toll-like receptor 3 (TLR3), and the cytoplasmic receptors, i.e., retinoic acid inducible gene-1 (RIG-1), melanoma differentiation-associated protein 5 (MDA-5), and protein kinase R (PRK) (Berke et al., 2013; Konat, 2016).

PIC preferentially binds toTLR3, which results in dimerization of the receptor and recruitment of the adaptor protein Toll-interleukin-1 (IL-1) receptor (TIR) domain-containing adaptor protein interferon- $\beta$ (TRIF) to the TIR domain of TLR3 (Perales-Linares and Navas-Martin, 2013). Activation of TLR3 initiates the antiviral-APR, characterized by the generation of a plethora of cytokines and additional inflammatory mediators, in particular the type 1 interferons INF $\alpha$ and INF $\beta$ (Guha-Thakurta and Majde, 1997). This APR instigates neurochemical changes within the brain, and ultimately sickness behavior.

Additionally, one needs to be cognizant that the peripheral PIC injection does not model viral infections. Rather, it models the initial response to viral infections, i.e., the APR. Directly studying the antiviral APR, as opposed to a viral infection provides multiple benefits. Firstly, the PIC-induced antiviral APR is an innate immune response and is produced regardless of the specifics of a virus, allowing us to more broadly understand the immune response to viral infections. Secondly, the APR has been repeatedly shown to underly the exacerbation of a patient's neurological symptoms.

As such, the PIC model offers a variety of advantages to study immune-to-brain communication. Thus, a single bolus injection of PIC into the peritoneal cavity results in a rapid production of inflammatory mediators that are quickly relayed to the brain induce the cerebral inflammatory response. PIC injected into the intraperitoneal cavity is rapidly degraded by RNases (Krasowska-Zoladek et al., 2007) and does not reach the circulation (Fil et al., 2011). Thus, the brain is exposed to a bolus stimulation solely by APR inflammatory mediators and is not 
compounded by viral particles in the circulation. Consequently, these features allow detailed kinetic analyses of both the systemic and cerebral responses.

\subsubsection{BLOOD CYTOKINES}

Peripheral PIC challenge induces significant yet transient elevation of serum levels of several key proinflammatory cytokines: interferon-beta (INF- $\beta$ ), interleukin-6 (IL-6), tumor necrosis factor-alpha (TNF $\alpha$ ), and interleukin-1 beta (IL-1 $\beta$ ) (Cunningham et al., 2007; Michalovicz and Konat, 2014). Serum levels rapidly peak at 3 hours post-PIC challenge, and promptly decline back to basal levels. All these cytokines are known to modulate neuronal activity (Schneider et al., 1998; Viviani et al., 2003; Schafers and Sorkin, 2008; Olmos and Llado, 2014), and to contribute to the induction of sickness behavior (Murray et al., 2015). Of note, the injection of

blood plasma from PIC-challenged animals into naïve mice mimics the cerebral response to PIC challenge (Fil et al., 2011), indicating that the response is mediated by blood-borne factors.

\subsubsection{BEHAVIOR}

As described above, intraperitoneal injection of PIC into mice results in sickness behavior (Guha-Thakurta and Majde, 1997; Cunningham et al., 2007; Michalovicz and Konat, 2014), which is consistent with the symptoms of viral infections in humans (Loftis et al., 2008; Nelligan et al., 2008). In mice, sickness behavior peaks at 6 hours following PIC injection, and subsides within 48 hours. These results are consistently seen across motivational tests including burrowing test, and locomotor activity examined by the open field and rearing tests (Cunningham et al., 2007; Konat et al., 2009; Michalovicz and Konat, 2014). Notably, the rearing test developed in the Konat lab provides the most sensitive assessment of PIC challenge-induced sickness behavior (Michalovicz and Konat, 2014). This test was routinely used in the present studies.

\subsubsection{HiPPOCAMPAL HYPEREXCITABILITY}

Peripheral PIC challenge exerts profound functional effects on neuronal circuitry in the brain. A stellar example of this is demonstrated by Kirschman et al. (2011) in which pretreatment with PIC exacerbated chemically induced seizures. As mentioned previously, peripheral viral infections increase the risk of seizures in the susceptible population (Tellez-Zenteno et al., 2005; Vezzani and Granata, 2005). In this experimental paradigm, PIC challenge 24 hours prior to seizure induction with the glutamate agonist, kainic acid (KA), dramatically augmented seizure severity and duration. This seizure hypersusceptibility persisted up to 72 hours post PIC 
challenge, indicating that a single PIC challenge results in a protracted hyperexcitability of neuronal networks in the brain.

Seizures result from an imbalance of excitatory (glutamatergic) and inhibitory (GABAergic) neurotransmission resulting in an overall hyperexcitable network. This imbalance can result from an increase in excitatory transmission, a decrease in inhibitory transmission, or both (During and Spencer, 1993; Bradford, 1995; Barker-Haliski and White, 2015). In line with enhanced neuronal activity following PIC challenge, our electrophysiological studies of the hippocampus, the ictal site of KA induced seizures, 24 hours after PIC demonstrated enhanced basal synaptic activity and plasticity (long term potentiation, LTP) compared to saline injected controls, two indicators of neuronal hyperexcitability (Hunsberger et al., 2016).

\subsubsection{EXTRACELLULAR GLUTAMATE}

Studies in anesthetized mice have demonstrated a profound increase in extracellular glutamate levels in the hippocampus 24 hour after peripheral PIC challenge (Hunsberger et al., 2016; Hunsberger et al., 2017)). The largest increase of 11-fold was observed in the dentate gyrus (DG), closely followed by 9.8-fold increase in the cornu ammonis 1 (CA1) and 5.8-fold increase in the cornu ammonis 3 (CA3)-Similar increase in the extracellular glutamate was observed in awake animals with implanted glutamate-sensing electrodes (Hunsberger et al., 2017). Moreover, increased extracellular glutamate positively correlated with seize severity and duration.

Although there was no differences in synaptic capacity to release glutamate as seen from potassium evoked release, PIC challenge decreased the paired-pulse facilitation (PPF), indicating increased probability of glutamate release (Hunsberger et al., 2016)lt is plausible that this discrepancy is due to potassium evoked release measuring the ceiling capacity for glutamate release rather than the more physiological levels demonstrated by PFF. Astrocytes are responsible for the uptake and recycling of glutamate via the excitatory amino acid transporters (EAATs). Astrocytes can also release glutamate through the cystine-glutamate antiporter (the system xc; Sxc), which exchanges extracellular cystine for intracellular glutamate. PIC challenge decreased the uptake of exogenously applied glutamate, and increased efflux of intracellular glutamate, indicating that both EAAT and Sxc might be responsible for the augmentation of extracellular glutamate. 


\subsubsection{Cerebral Gene expression}

Genome-wide transcriptome analysis of the hippocampus in response to PIC challenge revealed a total of 625 differentially expressed genes (DEGs) identified as dysregulated genes during 48 hours after PIC challenge (Michalovicz et al., 2015a). Unsurprisingly, this genomic reprogramming entails mostly genes related to the innate immune response, in particular cytokine, chemokine, and complement genes, as well as several genes related to neurotransmission.

1.3.5.1. Cytokines. The levels of cerebral messenger RNA (mRNA) encoding the proinflammatory cytokines INF $\beta$, IL-6, IL-1 $\beta$, and TNF $\alpha$ peaked within 3-6 hours post PIC challenge and returned to baseline at 48 hours (Cunningham et al., 2007; Fil et al., 2011; Michalovicz and Konat, 2014). Moreover, similar upregulation of cytokine mRNA was observed across major brain regions (Cunningham et al., 2007; Konat et al., 2009), indicating that the upregulation is global and not restricted to a specific anatomical structures.

Cytokines play an important role in the central nervous system by modulating neuronal transmission. Thus, IL-1 $\beta$ decreases calcium influx from cultured hippocampal neurons, and reduces presynaptic glutamate release (Murray et al., 1997) that creates functional deficit in LTP (Cunningham et al., 1996; MacManus et al., 2000; Zhou et al., 2006b; Zhou et al., 2006a). At high IL-1 $\beta$ concentrations, this impairment is further compounded post-synaptically by decreasing both spontaneous and evoked N-methyl-D-aspartate (NMDA) post-synaptic currents (Yang et al., 2005). TNF $\alpha$, which is constitutively expressed at low levels in both neurons and glial cells in vitro, also exhibits multiple neuromodulatory functions (Gahring et al., 1996; Stellwagen et al., 2005). Increased TNF $\alpha$ expression enhances excitatory neurotransmission via an exocytosis of $\alpha$-amino-3-hydroxy-5-methyl-4-isoxazolepropionic acid (AMPA) receptors while simultaneously diminishing inhibitory neurotransmission by removing gamma-aminobutyric acid (GABA) receptors from neuronal membranes (Stellwagen et al., 2005). Additionally, the constative nature of low levels of the cytokine allow for glial TNF $\alpha$ to modulate synaptic scaling (Beattie et al., 2002; Stellwagen et al., 2005). Short term application of TNF $\alpha$ to cultured astrocytes impairs glutamate uptake by downregulating glutamate transporters (Zou and Crews, 2005; Olmos and Llado, 2014), or by damaging these transporters via the generation of reactive oxygen species (Mir et al., 2009; Sandoval et al., 2018). 
1.3.5.2. Chemokines. The mRNA of a plethora of chemokines and their receptors are also significantly upregulated in the brain following PIC challenge (Fil et al., 2011; Michalovicz and Konat, 2014). CXCL1, CXCL9, CXCL10, and CXCL11 feature the highest upregulation by several thousand-folds over control (Fil et al., 2011; Michalovicz and Konat, 2014; Konat, 2016). The CCL2, CCL5, CXCL1 and CXLC2 genes are also upregulated, however only by several hundred-folds. Most chemokine mRNAs peak rapidly, within 3-6 hours, and several remain elevated even 72 hours post PIC challenge.

While the neuromodulatory functions of chemokines has not been thoroughly researched, there is mounting evidence that chemokines play an important role in both maintaining synaptic networks and altering them in disease. CXCL10 (inducible protein 10/IP-10) along with CXCL9, (monokine induced by gamma interferon/MIG) and CXCL11 (interferon-inducible T-cell alpha chemoattractant/TAC) bind to their cognate receptor, CXCR3. In C57BL/6 mice, however, CXCL11 is non-functional due to a point mutation (Groom and Luster, 2011a). Astrocytic production of CXCL10 enhanced long-term potentiation of the hippocampal networks (VIkolinsky et al., 2004). In vitro studies examining the effects of exogenous CXLC10 found its application to increase both spontaneous and evoked electrical activity as well as intracellular calcium levels (Nelson and Gruol, 2004; Sui et al., 2006). This increase in electrical activity was paralleled by decreased GABAergic and increased glutamatergic signaling (Cho et al., 2009; Tyagarajan et al., 2013; Zacchi et al., 2014; Battaglia et al., 2018).

CCL2, which signals through its cognate receptor CCR2, also increases neuronal activity. Acute exogenous CCL2 application to hippocampal slices increased postsynaptic excitatory potentials although no changes to LTP were observed (Gruol, 2016). Additionally, mice engineered to overexpress CCL2 in astrocytes exhibit similar results, with enhancements in short-term but not long-term potentiation (Nelson et al., 2011). Furthermore, CCL2 elicits a transient calcium influx in cultured neurons (Banisadr et al., 2005) and significantly reduces GABA-induced neuronal currents in spinal neurons (Gosselin et al., 2005).

1.3.5.3. Complement proteins. Transcriptomics analysis identified the complement pathway as the primary pathway upregulated by peripheral PIC challenge in the hippocampus (Michalovicz et al 2015). Quantitative real time polymerase chain reaction (qRT-PCR) revealed several complement genes, to be significantly upregulated following PIC challenge. These genes exhibited a rapid increase in expression, peaking at 24 hours post-PIC. The mRNAs encoding 
complement factor b (Cfb), C6, and C3 featured the highest peak upregulation, of 53-, 40-, and 12-fold over control, respectively.

Recent studies have revealed a critical role of the complement system during brain development by tagging overabundant synapses for phagocytosis by microglia (Stevens et al., 2007; Schafer et al., 2012; Stephan et al., 2012). Additionally, aberrant activation of the complement system in neurological disorders, such as epilepsy and AD has been hypothesized to be responsible for synapse loss (Zhou et al., 2008; Fonseca et al., 2011; Hong et al., 2016b).

\subsection{DISSERTATION AIMS}

As discussed above, activation of the innate immune system in the periphery induces profound molecular and functional changes in the brain. In particular, the antiviral APR exacerbates neurological conditions resulting likely from a protracted neuronal hyperexcitability. However, no mediators responsible for the induction of hyperexcitability have been identified. The robust upregulation of inflammatory genes in the brain discussed above suggests that their protein products may mediate the development of hyperexcitability in response to PIC challenge. The aim of this dissertation was to test this possibility by characterizing expression of these genes at the protein level. The ultimate long-term goal of this research endeavor is to provide a springboard for the development of novel therapeutics that would target cerebral pathways to prevent/ameliorate the exacerbation of neurological symptoms without hampering the resolution of systemic infections. 


\subsection{REFERENCES}

Adelman JS, Martin LB (2009) Vertebrate sickness behaviors: Adaptive and integrated neuroendocrine immune responses. Integr Comp Biol 49:202-214.

Andersen O, Lygner PE, Bergstrom T, Andersson M, Vahlne A (1993) Viral infections trigger multiple sclerosis relapses: a prospective seroepidemiological study. J Neurol 240:417422.

Asensio VC, Campbell IL (1999) Chemokines in the CNS: plurifunctional mediators in diverse states. Trends Neurosci 22:504-512.

Bajova H, Nelson TE, Gruol DL (2008) Chronic CXCL10 alters the level of activated ERK1/2 and transcriptional factors CREB and NF-kappaB in hippocampal neuronal cell culture. J Neuroimmunol 195:36-46.

Banisadr G, Gosselin RD, Mechighel P, Rostene W, Kitabgi P, Melik Parsadaniantz S (2005) Constitutive neuronal expression of CCR2 chemokine receptor and its colocalization with neurotransmitters in normal rat brain: functional effect of MCP-1/CCL2 on calcium mobilization in primary cultured neurons. J Comp Neurol 492:178-192.

Barker-Haliski M, White HS (2015) Glutamatergic Mechanisms Associated with Seizures and Epilepsy. Cold Spring Harb Perspect Med 5:a022863.

Battaglia S, Renner M, Russeau M, Come E, Tyagarajan SK, Levi S (2018) Activity-Dependent Inhibitory Synapse Scaling Is Determined by Gephyrin Phosphorylation and Subsequent Regulation of GABAA Receptor Diffusion. eNeuro 5.

Baumann H, Gauldie J (1994) The acute phase response. Immunol Today 15:74-80.

Beattie EC, Stellwagen D, Morishita W, Bresnahan JC, Ha BK, Von Zastrow M, Beattie MS, Malenka RC (2002) Control of synaptic strength by glial TNFalpha. Science 295:22822285.

Berke IC, Li Y, Modis Y (2013) Structural basis of innate immune recognition of viral RNA. Cell Microbiol 15:386-394.

Bradford HF (1995) Glutamate, GABA and epilepsy. Prog Neurobiol 47:477-511.

Brugger F, Erro R, Balint B, Kagi G, Barone P, Bhatia KP (2015) Why is there motor deterioration in Parkinson's disease during systemic infections-a hypothetical view. NPJ Parkinsons Dis 1:15014.

Buljevac D, Flach HZ, Hop WC, Hijdra D, Laman JD, Savelkoul HF, van Der Meche FG, van Doorn PA, Hintzen RQ (2002) Prospective study on the relationship between infections and multiple sclerosis exacerbations. Brain 125:952-960.

Cavaillon JM (2001) Pro- versus anti-inflammatory cytokines: myth or reality. Cell Mol Biol (Noisy-le-grand) 47:695-702.

Cho J, Nelson TE, Bajova H, Gruol DL (2009) Chronic CXCL10 alters neuronal properties in rat hippocampal culture. J Neuroimmunol 207:92-100.

Colvin RA, Campanella GS, Sun J, Luster AD (2004) Intracellular domains of CXCR3 that mediate CXCL9, CXCL10, and CXCL11 function. J Biol Chem 279:30219-30227.

Cray C, Zaias J, Altman NH (2009) Acute phase response in animals: a review. Comp Med 59:517-526.

Cunningham AJ, Murray CA, O'Neill LA, Lynch MA, O'Connor JJ (1996) Interleukin-1 beta (IL-1 beta) and tumour necrosis factor (TNF) inhibit long-term potentiation in the rat dentate gyrus in vitro. Neurosci Lett 203:17-20.

Cunningham C, Campion S, Teeling J, Felton L, Perry VH (2007) The sickness behaviour and CNS inflammatory mediator profile induced by systemic challenge of mice with synthetic double-stranded RNA (poly I:C). Brain Behav Immun 21:490-502.

Curnock AP, Logan MK, Ward SG (2002) Chemokine signalling: pivoting around multiple phosphoinositide 3-kinases. Immunology 105:125-136. 
Dantzer R (2006) Cytokine, sickness behavior, and depression. Neurol Clin 24:441-460.

Dantzer R (2009) Cytokine, sickness behavior, and depression. Immunol Allergy Clin North Am 29:247-264.

During MJ, Spencer DD (1993) Extracellular hippocampal glutamate and spontaneous seizure in the conscious human brain. Lancet 341:1607-1610.

Edwards S, Zvartau M, Clarke H, Irving W, Blumhardt LD (1998) Clinical relapses and disease activity on magnetic resonance imaging associated with viral upper respiratory tract infections in multiple sclerosis. J Neurol Neurosurg Psychiatry 64:736-741.

Fil D, Borysiewicz E, Konat GW (2011) A broad upregulation of cerebral chemokine genes by peripherally-generated inflammatory mediators. Metab Brain Dis 26:49-59.

Fleshner M, Goehler LE, Hermann J, Relton JK, Maier SF, Watkins LR (1995) Interleukin-1 beta induced corticosterone elevation and hypothalamic NE depletion is vagally mediated. Brain Res Bull 37:605-610.

Fonseca MI, Chu SH, Berci AM, Benoit ME, Peters DG, Kimura Y, Tenner AJ (2011) Contribution of complement activation pathways to neuropathology differs among mouse models of Alzheimer's disease. J Neuroinflammation 8:4.

Gahring LC, Carlson NG, Kulmar RA, Rogers SW (1996) Neuronal expression of tumor necrosis factor alpha in the murine brain. Neuroimmunomodulation 3:289-303.

Ganong WF (2000) Circumventricular organs: definition and role in the regulation of endocrine and autonomic function. Clin Exp Pharmacol Physiol 27:422-427.

George J, Bleasdale S, Singleton SJ (1997) Causes and prognosis of delirium in elderly patients admitted to a district general hospital. Age Ageing 26:423-427.

Gosselin RD, Varela C, Banisadr G, Mechighel P, Rostene W, Kitabgi P, Melik-Parsadaniantz S (2005) Constitutive expression of CCR2 chemokine receptor and inhibition by MCP1/CCL2 of GABA-induced currents in spinal cord neurones. J Neurochem 95:1023-1034.

Groom JR, Luster AD (2011) CXCR3 ligands: redundant, collaborative and antagonistic functions. Immunol Cell Biol 89:207-215.

Gruol DL (2016) Impact of Increased Astrocyte Expression of IL-6, CCL2 or CXCL10 in Transgenic Mice on Hippocampal Synaptic Function. Brain Sci 6.

Guha-Thakurta N, Majde JA (1997) Early induction of proinflammatory cytokine and type I interferon mRNAs following Newcastle disease virus, poly [rl:rC], or low-dose LPS challenge of the mouse. J Interferon Cytokine Res 17:197-204.

Hart BL (1988) Biological basis of the behavior of sick animals. Neurosci Biobehav Rev 12:123137.

Holdsworth SR, Gan PY (2015) Cytokines: Names and Numbers You Should Care About. Clin J Am Soc Nephrol 10:2243-2254.

Holmes C (2013) Review: systemic inflammation and Alzheimer's disease. Neuropathol Appl Neurobiol 39:51-68.

Holmes C, El-Okl M, Williams AL, Cunningham C, Wilcockson D, Perry VH (2003) Systemic infection, interleukin 1 beta, and cognitive decline in Alzheimer's disease. J Neurol Neurosurg Psychiatry 74:788-789.

Hong S, Beja-Glasser VF, Nfonoyim BM, Frouin A, Li S, Ramakrishnan S, Merry KM, Shi Q, Rosenthal A, Barres BA, Lemere CA, Selkoe DJ, Stevens B (2016) Complement and microglia mediate early synapse loss in Alzheimer mouse models. Science 352:712-716.

Huang CY, Yang HI, Chen SD, Shaw FZ, Yang DI (2008) Protective effects of lipopolysaccharide preconditioning against nitric oxide neurotoxicity. J Neurosci Res 86:1277-1289.

Hunsberger HC, Konat GW, Reed MN (2017) Peripheral viral challenge elevates extracellular glutamate in the hippocampus leading to seizure hypersusceptibility. J Neurochem 141:341-346. 
Hunsberger HC, Wang D, Petrisko TJ, Alhowail A, Setti SE, Suppiramaniam V, Konat GW, Reed MN (2016) Peripherally restricted viral challenge elevates extracellular glutamate and enhances synaptic transmission in the hippocampus. J Neurochem 138:307-316.

Jacobs BL, Langland JO (1996) When two strands are better than one: the mediators and modulators of the cellular responses to double-stranded RNA. Virology 219:339-349.

Jain S, Gautam V, Naseem S (2011) Acute-phase proteins: As diagnostic tool. J Pharm Bioallied Sci 3:118-127.

Kirschman LT, Borysiewicz E, Fil D, Konat GW (2011) Peripheral immune challenge with dsRNA enhances kainic acid-induced status epilepticus. Metab Brain Dis 26:91-93.

Konat G (2016) Cerebral Response to Peripheral Challenge with a Viral Mimetic. Neurochemical research 41:144-155.

Konat GW, Borysiewicz E, Fil D, James I (2009) Peripheral challenge with double-stranded RNA elicits global up-regulation of cytokine gene expression in the brain. J Neurosci Res 87:1381-1388.

Krasowska-Zoladek A, Banaszewska M, Kraszpulski M, Konat GW (2007) Kinetics of inflammatory response of astrocytes induced by TLR 3 and TLR4 ligation. J Neurosci Res 85:205-212.

Kushner I, Rzewnicki DL (1994) The acute phase response: general aspects. Baillieres Clin Rheumatol 8:513-530.

Learoyd AE et al. (2017) Infections Up to 76 Days After Stroke Increase Disability and Death. Transl Stroke Res 8:541-548.

Legler DF, Thelen M (2018) New insights in chemokine signaling. F1000Res 7:95.

Libbey JE, Fujinami RS (2010) Potential triggers of MS. Results Probl Cell Differ 51:21-42.

Loftis JM, Huckans M, Ruimy S, Hinrichs DJ, Hauser P (2008) Depressive symptoms in patients with chronic hepatitis $\mathrm{C}$ are correlated with elevated plasma levels of interleukin-1beta and tumor necrosis factor-alpha. Neurosci Lett 430:264-268.

Lv Z, Liu C, Zhai M, Zhang Q, Li J, Zheng F, Peng M (2018) LPS Pretreatment Attenuates Cerebral Ischaemia/Reperfusion Injury by Inhibiting Inflammation and Apoptosis. Cell Physiol Biochem 45:2246-2256.

MacManus A, Ramsden M, Murray M, Henderson Z, Pearson HA, Campbell VA (2000) Enhancement of $(45) \mathrm{Ca}(2+)$ influx and voltage-dependent $\mathrm{Ca}(2+)$ channel activity by beta-amyloid-(1-40) in rat cortical synaptosomes and cultured cortical neurons. Modulation by the proinflammatory cytokine interleukin-1beta. J Biol Chem 275:47134718.

Mardiguian S, Ladds E, Turner R, Shepherd H, Campbell SJ, Anthony DC (2017) The contribution of the acute phase response to the pathogenesis of relapse in chronicrelapsing experimental autoimmune encephalitis models of multiple sclerosis. $\mathrm{J}$ Neuroinflammation 14:196.

McCusker RH, Kelley KW (2013) Immune-neural connections: how the immune system's response to infectious agents influences behavior. J Exp Biol 216:84-98.

Medzhitov R (2001) Toll-like receptors and innate immunity. Nat Rev Immunol 1:135-145.

Michalovicz LT, Konat GW (2014) Peripherally restricted acute phase response to a viral mimic alters hippocampal gene expression. Metab Brain Dis 29:75-86.

Michalovicz LT, Lally B, Konat GW (2015) Peripheral challenge with a viral mimic upregulates expression of the complement genes in the hippocampus. J Neuroimmunol 285:137142.

Mir M, Asensio VJ, Tolosa L, Gou-Fabregas M, Soler RM, Llado J, Olmos G (2009) Tumor necrosis factor alpha and interferon gamma cooperatively induce oxidative stress and motoneuron death in rat spinal cord embryonic explants. Neuroscience 162:959-971. 
Murray AM, Levkoff SE, Wetle TT, Beckett L, Cleary PD, Schor JD, Lipsitz LA, Rowe JW, Evans DA (1993) Acute delirium and functional decline in the hospitalized elderly patient. J Gerontol 48:M181-186.

Murray C, Griffin EW, O'Loughlin E, Lyons A, Sherwin E, Ahmed S, Stevenson NJ, Harkin A, Cunningham C (2015) Interdependent and independent roles of type I interferons and IL6 in innate immune, neuroinflammatory and sickness behaviour responses to systemic poly I:C. Brain Behav Immun 48:274-286.

Murray CA, McGahon B, McBennett S, Lynch MA (1997) Interleukin-1 beta inhibits glutamate release in hippocampus of young, but not aged, rats. Neurobiol Aging 18:343-348.

Nee LE, Lippa CF (1999) Alzheimer's disease in 22 twin pairs--13-year follow-up: hormonal, infectious and traumatic factors. Dement Geriatr Cogn Disord 10:148-151.

Nelligan JA, Loftis JM, Matthews AM, Zucker BL, Linke AM, Hauser P (2008) Depression comorbidity and antidepressant use in veterans with chronic hepatitis $\mathrm{C}$ : results from a retrospective chart review. J Clin Psychiatry 69:810-816.

Nelson TE, Gruol DL (2004) The chemokine CXCL10 modulates excitatory activity and intracellular calcium signaling in cultured hippocampal neurons. J Neuroimmunol 156:7487.

Nelson TE, Hao C, Manos J, Ransohoff RM, Gruol DL (2011) Altered hippocampal synaptic transmission in transgenic mice with astrocyte-targeted enhanced CCL2 expression. Brain Behav Immun 25 Suppl 1:S106-119.

Olmos G, Llado J (2014) Tumor necrosis factor alpha: a link between neuroinflammation and excitotoxicity. Mediators Inflamm 2014:861231.

Packard AE, Hedges JC, Bahjat FR, Stevens SL, Conlin MJ, Salazar AM, Stenzel-Poore MP (2012) Poly-IC preconditioning protects against cerebral and renal ischemia-reperfusion injury. J Cereb Blood Flow Metab 32:242-247.

Panitch HS (1994) Influence of infection on exacerbations of multiple sclerosis. Ann Neurol 36 Suppl:S25-28.

Perales-Linares R, Navas-Martin S (2013) Toll-like receptor 3 in viral pathogenesis: friend or foe? Immunology 140:153-167.

Quan N, Banks WA (2007) Brain-immune communication pathways. Brain Behav Immun 21:727-735.

Reaux-Le Goazigo A, Van Steenwinckel J, Rostene W, Melik Parsadaniantz S (2013) Current status of chemokines in the adult CNS. Prog Neurobiol 104:67-92.

Sandoval R, Lazcano P, Ferrari F, Pinto-Pardo N, Gonzalez-Billault C, Utreras E (2018) TNFalpha Increases Production of Reactive Oxygen Species through Cdk5 Activation in Nociceptive Neurons. Front Physiol 9:65.

Schafer DP, Lehrman EK, Kautzman AG, Koyama R, Mardinly AR, Yamasaki R, Ransohoff RM, Greenberg ME, Barres BA, Stevens B (2012) Microglia sculpt postnatal neural circuits in an activity and complement-dependent manner. Neuron 74:691-705.

Schafers M, Sorkin L (2008) Effect of cytokines on neuronal excitability. Neurosci Lett 437:188193.

Schneider H, Pitossi F, Balschun D, Wagner A, del Rey A, Besedovsky HO (1998) A neuromodulatory role of interleukin-1 beta in the hippocampus. Proc Natl Acad Sci U S A 95:7778-7783.

Shakhar K, Shakhar G (2015) Why Do We Feel Sick When Infected--Can Altruism Play a Role? PLoS Biol 13:e1002276.

Stellwagen D, Beattie EC, Seo JY, Malenka RC (2005) Differential regulation of AMPA receptor and GABA receptor trafficking by tumor necrosis factor-alpha. J Neurosci 25:3219-3228.

Stephan AH, Barres BA, Stevens B (2012) The complement system: an unexpected role in synaptic pruning during development and disease. Annu Rev Neurosci 35:369-389. 
Stevens B, Allen NJ, Vazquez LE, Howell GR, Christopherson KS, Nouri N, Micheva KD, Mehalow AK, Huberman AD, Stafford B, Sher A, Litke AM, Lambris JD, Smith SJ, John SW, Barres BA (2007) The classical complement cascade mediates CNS synapse elimination. Cell 131:1164-1178.

Sui Y, Stehno-Bittel L, Li S, Loganathan R, Dhillon NK, Pinson D, Nath A, Kolson D, Narayan O, Buch S (2006) CXCL10-induced cell death in neurons: role of calcium dysregulation. Eur J Neurosci 23:957-964.

Takeuchi O, Akira S (2010) Pattern recognition receptors and inflammation. Cell 140:805-820.

Tellez-Zenteno JF, Matijevic S, Wiebe S (2005) Somatic comorbidity of epilepsy in the general population in Canada. Epilepsia 46:1955-1962.

Turner MD, Nedjai B, Hurst T, Pennington DJ (2014) Cytokines and chemokines: At the crossroads of cell signalling and inflammatory disease. Biochim Biophys Acta 1843:2563-2582.

Turner RC, Naser ZJ, Lucke-Wold BP, Logsdon AF, Vangilder RL, Matsumoto RR, Huber JD, Rosen CL (2017) Single low-dose lipopolysaccharide preconditioning: neuroprotective against axonal injury and modulates glial cells. Neuroimmunol Neuroinflamm 4:6-15.

Tyagarajan SK, Ghosh H, Yevenes GE, Imanishi SY, Zeilhofer HU, Gerrits B, Fritschy JM (2013) Extracellular signal-regulated kinase and glycogen synthase kinase 3beta regulate gephyrin postsynaptic aggregation and GABAergic synaptic function in a calpain-dependent mechanism. J Biol Chem 288:9634-9647.

Verrotti A, Tocco AM, Coppola GG, Altobelli E, Chiarelli F (2009) Afebrile benign convulsions with mild gastroenteritis: a new entity? Acta Neurol Scand 120:73-79.

Vezzani A, Granata T (2005) Brain inflammation in epilepsy: experimental and clinical evidence. Epilepsia 46:1724-1743.

Viviani B, Bartesaghi S, Gardoni F, Vezzani A, Behrens MM, Bartfai T, Binaglia M, Corsini E, Di Luca M, Galli CL, Marinovich M (2003) Interleukin-1beta enhances NMDA receptormediated intracellular calcium increase through activation of the Src family of kinases. J Neurosci 23:8692-8700.

Vlkolinsky R, Siggins GR, Campbell IL, Krucker T (2004) Acute exposure to CXC chemokine ligand 10, but not its chronic astroglial production, alters synaptic plasticity in mouse hippocampal slices. J Neuroimmunol 150:37-47.

Wang X, Lupardus P, Laporte SL, Garcia KC (2009) Structural biology of shared cytokine receptors. Annu Rev Immunol 27:29-60.

Watkins LR, Maier SF, Goehler LE (1995) Cytokine-to-brain communication: a review \& analysis of alternative mechanisms. Life Sci 57:1011-1026.

Weber F, Wagner V, Rasmussen SB, Hartmann R, Paludan SR (2006) Double-stranded RNA is produced by positive-strand RNA viruses and DNA viruses but not in detectable amounts by negative-strand RNA viruses. J Virol 80:5059-5064.

Westendorp WF, Nederkoorn PJ, Vermeij JD, Dijkgraaf MG, van de Beek D (2011) Post-stroke infection: a systematic review and meta-analysis. BMC Neurol 11:110.

Yang S, Liu ZW, Wen L, Qiao HF, Zhou WX, Zhang YX (2005) Interleukin-1beta enhances NMDA receptor-mediated current but inhibits excitatory synaptic transmission. Brain Res 1034:172-179.

Yu S, Liu X, Zhang N, Yang S, Mao C, Feng S, Lu H (2018) Protection of Lipopolysaccharide (LPS) Preconditioning against Endotoxin-Induced Uveitis (EIU) in Rats is Associated with Overexpression of Interleukin-1 Receptor-Associated Kinase M (IRAK-M). Ocul Immunol Inflamm 26:943-950.

Zacchi P, Antonelli R, Cherubini E (2014) Gephyrin phosphorylation in the functional organization and plasticity of GABAergic synapses. Front Cell Neurosci 8:103.

Zhang JM, An J (2007) Cytokines, inflammation, and pain. Int Anesthesiol Clin 45:27-37. 
Zheng KS, Dorfman BJ, Christos PJ, Khadem NR, Henchcliffe C, Piboolnurak P, Nirenberg MJ (2012) Clinical characteristics of exacerbations in Parkinson disease. Neurologist 18:120-124.

Zhou C, Ye HH, Wang SQ, Chai Z (2006a) Interleukin-1beta regulation of N-type Ca2+ channels in cortical neurons. Neurosci Lett 403:181-185.

Zhou C, Tai C, Ye HH, Ren X, Chen JG, Wang SQ, Chai Z (2006b) Interleukin-1beta downregulates the L-type Ca2+ channel activity by depressing the expression of channel protein in cortical neurons. J Cell Physiol 206:799-806.

Zhou J, Fonseca MI, Pisalyaput K, Tenner AJ (2008) Complement C3 and C4 expression in C1q sufficient and deficient mouse models of Alzheimer's disease. J Neurochem 106:2080-2092.

Zou JY, Crews FT (2005) TNF alpha potentiates glutamate neurotoxicity by inhibiting glutamate uptake in organotypic brain slice cultures: neuroprotection by NF kappa B inhibition. Brain Res 1034:11-24. 


\section{CHAPTER 2: PERIPHERAL VIRAL CHALLENGE TRIGGERS HIPPOCAMPAL PRODUCTION OF INFLAMMATORY PROTEINS}

Original Article: Metabolic Brain Disease (2017) vol. 32, issue 4, pgs. 1249-1254

Tiffany Petrisko, Gregory Konat

Department of Neurobiology and Anatomy, West Virginia University School of Medicine, 1 Medical Center Dr., Morgantown, WV 26506, USA

\subsection{ABSTRACT}

Peripheral viral infections increase seizure propensity and intensity in susceptible individuals. We have modeled this comorbidity by demonstrating that intraperitoneal (ip) injection of the conventional viral mimetic, polyinosinic-polycytidylic acid (PIC), renders the brain hypersusceptible to seizures induced by kainic acid (KA). At the molecular level, the hippocampus, which is the ictal site of KA-induced seizures, exhibits upregulated expression of messages encoding several inflammatory genes. Here, we profiled temporal expression of these genes at the protein level. Briefly, eight-week old female C57BL/6 mice were ip injected with $12 \mathrm{mg} / \mathrm{kg}$ of PIC and inflammatory proteins were quantified in the hippocampus and blood by ELISA. We found a robust but transient increase in blood concentration of IL-6, CXCL10, CCL2, CXCL9, CCL7 and CCL12 six hours after PIC challenge. CXCL1, IL1 $\beta$, TNF $\alpha$ and CXCL2 featured a moderate increase. However, only four chemokines were increased in the hippocampus. CXCL10 showed the highest increase 6-12 $\mathrm{h}$ after PIC challenge, and its level dwindled to the baseline by $48 \mathrm{~h}$. CXCL1, CXCl9 and CXCL2 were also transiently elevated but their maximal values were by an order of magnitude lower than the values for CXCL10. These results indicate that CXCL10 is the primary inflammatory protein generated in the hippocampus in response to PIC challenge, and that this chemokine may drive the development of seizure hypersusceptibility. In addition, the hippocampus featured a protracted increase in the levels of anaphylatoxins $\mathrm{C} 3 \mathrm{a}$ and $\mathrm{C} 5 \mathrm{a}$, indicating the activation of the complement cascades. 


\subsection{INTRODUCTION}

Peripheral microbial infections have been recognized as important comorbid factors that increase the frequency and severity of seizures in susceptible individuals (Tellez-Zenteno et al., 2005; Scheid and Teich, 2007; Verrotti et al., 2009). Peripherally-instigated seizure hypersusceptibility is believed to be triggered by the acute phase reaction (APR), i.e., the initial response of the host aimed at containing the spread of infection. APR entails a fulminant generation of cytokines and other inflammatory mediators often referred to as "cytokine storm". These blood-borne mediators are relayed to the brain and induce inflammatory response in the cerebral parenchyma. The resultant neuroinflammatory milieu modifies the activity of neuronal networks and alters cerebral function (Dantzer, 2006; Dantzer and Kelley, 2007; Quan and Banks, 2007). In particular, the inflammatory agents may decrease inhibitory inputs, increase excitatory inputs, or both, resulting in hyperexcitability of neuronal networks, a hallmark of seizures. However, the pertinent agents and the underlying cellular/molecular mechanisms have not been explored in detail.

We have developed a preclinical murine model of seizure hypersusceptibility induced by peripheral viral challenge. In this model, APR induced by intraperitoneal (i.p.) injection of a viral mimetic, polyinosinic-polycytidylic acid (PIC), renders the brain hypersusceptible to kainic acid (KA)-induced seizures as seen from a robust increase in the intensity and duration of status epilepticus (Kirschman et al., 2011; Hunsberger et al., 2017). This hypersusceptibility is protracted for three days after PIC challenge (Michalovicz and Konat, 2014). This behavioral phenotype is underscored by profound hyperexcitability of neuronal networks in the hippocampus (Hunsberger et al., 2016), the ictal site of KA-induced seizures (Ben-Ari and Cossart, 2000). In a quest to identify molecular substrates of seizue hypersusceptibility, we profiled hipocampal gene expression. We found PIC challenge to induce an extensive genomic reprograming as seen from the dysregulated expression of 625 genes at the mRNA level (Michalovicz and Konat, 2014; Michalovicz et al., 2015b). The upregulated genes include genes encoding several chemokines and cytokines. Generally, the upregulation of respective mRNAs peaks between three and six hours following the PIC challenge. The $C x c / 11, C x c / 10$ and $C x c / 9$ genes feature the highest upregulation reaching the maxima of several thousand over the control values before gradually subsiding to the basal level. Other chemokine genes in descending order of upregulation are: $\mathrm{Cxc} / 1, \mathrm{Cxc} / 2, \mathrm{Cc} / 12, \mathrm{Ccl} 2, \mathrm{Cc} / 7$ and $\mathrm{Cc} / 5$. Also, the genes encoding cytokines with known ictogenic activity, i.e., IL-1 $\beta$, IL-6 and TNF $\alpha$ are modestly 
upregulated. In addition, PIC challenge significantly upregulates several genes within the complement pathway.

The present study was undertaken to parse a plausible involvement of inflammatory genes in the development of seizure hypersusceptible phenotype induced by PIC challenge. We selected hippocampal genes that feature upregulation at the message level, and profiled their expression at the protein level over the time period of seizure hypersusceptibility. We also determined temporal fluctuations of the respective proteins in the blood.

\subsection{METHODS}

\subsubsection{ANIMALS}

Eight-week old female C57BL/6J mice obtained from Charles River Laboratories (Wilmington, MA) were housed under 12-h light/dark conditions (lights on at 6 am) and fed ad libitum.

Peripheral APR was induced by a single i.p. injection of $12 \mathrm{mg} / \mathrm{kg}$ of ultrapure PIC (Invivogen, San Diego, CA) in $100 \mu$ l of saline. Mice injected with equivolume saline served as controls. To verify successful PIC injection, the development of sickness behavior was assessed after $6 \mathrm{~h}$ by the rearing test (Michalovicz and Konat, 2014). All procedures were approved by the West Virginia University Animal Care and Use Committee and conducted in compliance with the guidelines published in the NIH Guide for the Care and Use of Laboratory Animals.

\subsubsection{ELISA}

At different time points after PIC challenge, mice were deeply anesthetized by i.p. injection of 65 $\mathrm{mg} / \mathrm{ml}$ of pentobarbital (Beauthanasia, Patterson Veterinary, Devens, MA), and sacrificed by pneumothorax. Blood was quickly collected by heart puncture, and the animals were perfused transaortally with saline. Blood was allowed to coagulate overnight at $4^{\circ} \mathrm{C}$, and centrifuged at $3,000 \mathrm{rpm}$ for 10 minutes. Serum was aspirated and stored at $-20^{\circ} \mathrm{C}$. Both hippocampi were dissected and disintegrated in the lysis buffer $(50 \mathrm{mM}$ Tris- $\mathrm{HCl}, 150 \mathrm{mM} \mathrm{NaCl}, 2 \mathrm{mM}$ EDTA, 1\% Triton X-100, 0.5\% NP-40 and a protease inhibitor, $\mathrm{pH} 7.5$ ) by sonication. Complete Protease Inhibitor Cocktail was procured from Roche Diagnostics (Mannheim, Germany), and all other chemicals were from Sigma-Aldrich (St. Louis, MO). The homogenates were spun at 14,000 rpm for $20 \mathrm{~min}$ and the supernatants were aspirated and stored at $-20^{\circ} \mathrm{C}$. Inflammatory proteins were quantified by ELISA according to manufacturers' instructions. CXCL1, CXCL2, CXCL9, CXCL10, TNF- $\alpha$, and IL-1 $\beta$ ELISA kits were obtained from R\&D Systems, Inc. (Minneapolis, 
MN), while CCL2, CCL5, CCL7, CCL12, CfB, C3, C3a, C8b, and C9 ELISA Kits were from LifeSpan Biosciences (Seattle, WA). ELISA kits for C5a and IL-6 were purchased from Abcam (Cambridge, MA).

\subsubsection{PROTEIN DETERMINATION}

Total protein was measured in serum and tissue homogenates by ABC method (BioRad, Hercules, CA) using bovine serum albumin as a standard. The levels of inflammatory proteins determined by ELISA were normalized to the amount of total protein in samples.

\subsubsection{STATISTICAL ANALYSIS}

The values from PIC-injected vs. saline-injected mice were analyzed by one-way ANOVA followed by Bonferroni post-hoc test and presented as means \pm SE. Differences between groups were considered significant at $P \leq 0.05$.

\subsection{RESULTS}

APR induced by peripheral PIC challenge transiently upregulates hippocampal expression of messages encoding several chemokines (Michalovicz and Konat, 2014). Most of these chemokines surged transiently in the blood with a maximum attained $6 \mathrm{~h}$ after PIC injection (Fig. 1). CXCL10 reached the highest values $[F(5,14)=38.74, p<0.001]$, and was followed by CCL2 $[F(6,15)=21.67, p<0.001], \operatorname{CXCL9}[F(6,21)=34.59, p<0.001], \operatorname{CCL} 7[F(6,10)=12.76, p<0.001]$, $\operatorname{CCL} 12[F(6,14)=27.82, p<0.001], \operatorname{CXCL} 1[F(5,14)=39.52, p<0.001]$ and $\operatorname{CXCL} 2[F(5$, $14)=25.65, p<0.001]$. The concentration of all these species returned to control level by $48 \mathrm{~h}$ or sooner. Blood concentration of CCL5 was not affected by PIC challenge $[F(6,12)=1.30$, $\mathrm{p}=0.326$ ]. CXCL11 is inactive in C57BL/6 mice due to a null mutation (PubMed accession numbers NT_109320 and AK050012.1), and was not measured.

As shown in Fig. 1, CXCL10 also featured the highest generation in the hippocampus $12 \mathrm{~h}$ after $\mathrm{PIC}$ challenge $[\mathrm{F}(6,32)=44.96, \mathrm{p}<0.001]$. Its levels dropped by $45 \%$ at $24 \mathrm{~h}$, and returned to the baseline at $48 \mathrm{~h}$. The levels of $\operatorname{CXCL} 1[\mathrm{~F}(6,33)=38.50, p<0.001], \operatorname{CXCL9}[\mathrm{F}(6,32)=20.79$, $p<0.001]$ and $C X C L 2[F(6,29)=28.85, p<0.001]$ were also increased, although to a lesser extent. Unlike CXCL10, the levels of CXCL1, CXCL9 and CXCL2 rapidly declined to the baseline by $24 \mathrm{~h}$ after PIC challenge. Hippocampal concentrations of CCL2 $[F(6,13)=1.86$, 
$\mathrm{p}=0.165], \operatorname{CCL} 5[\mathrm{~F}(6,12)=0.59, \mathrm{p}=0.733], \operatorname{CCL} 7[\mathrm{~F}(6,12)=1.12, \mathrm{p}=0.406]$ and $\mathrm{CCL} 12$ [F(6, $14)=1.08, p=0.42]$ were not altered by PIC challenge.
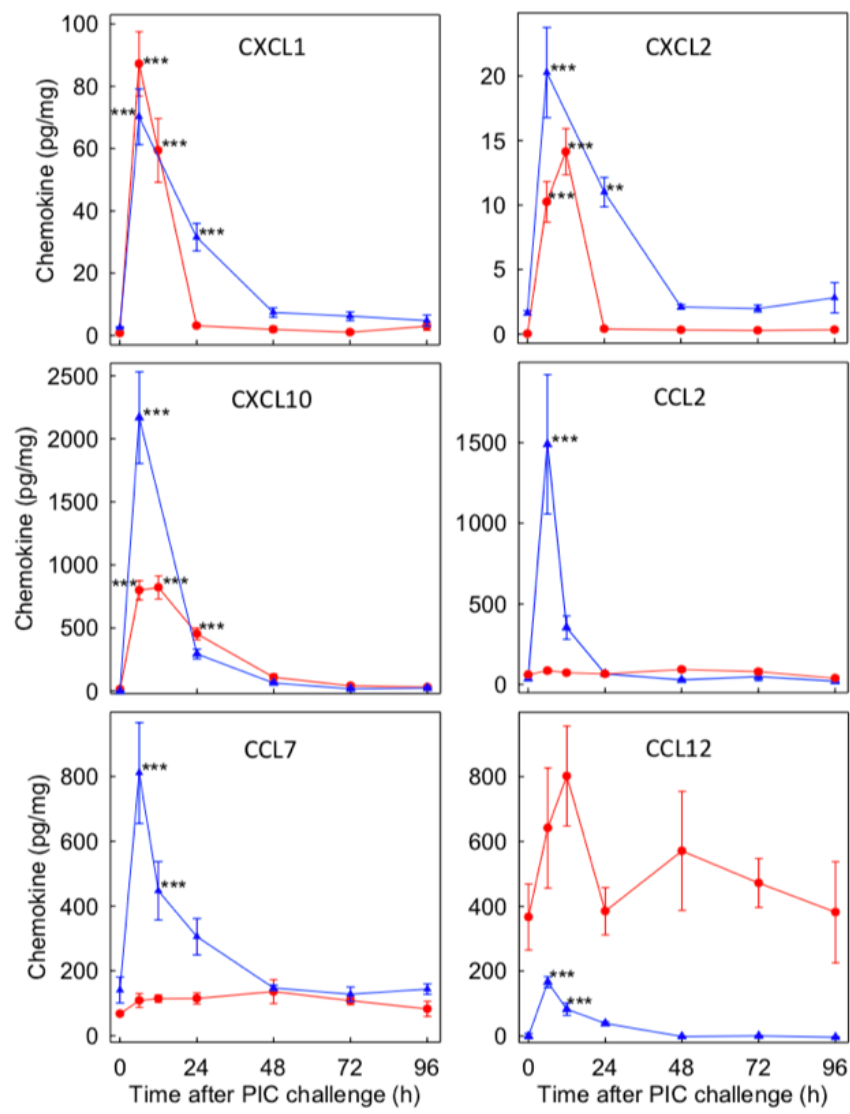
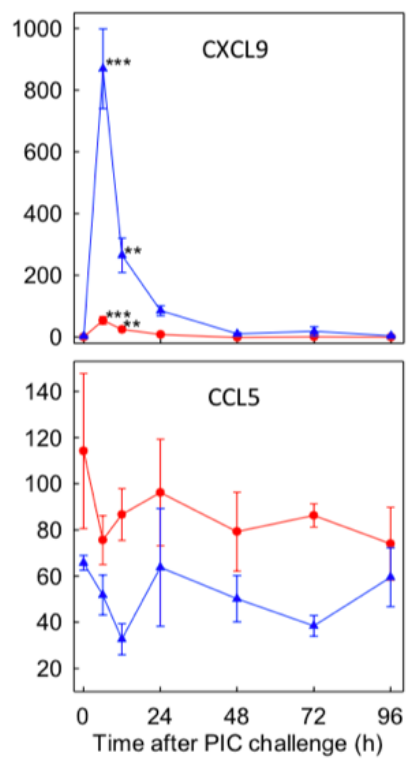

Figure 1. Production of chemokines induced by peripheral PIC challenge. Mice were intraperitoneally injected with $12 \mathrm{mg} / \mathrm{kg}$ of PIC. Saline-injected mice served as respective controls. At different time points, blood (blue triangles) and hippocampal (red circles) levels of the chemokines were quantified by ELISA. The results are expressed as pg of

chemokines per mg of total tissue protein. Symbols represent means \pm SEM from 4-7 animals. Asterisks denote significant differences from saline-injected controls $\left({ }^{* *} p \leq 0.01 ;{ }^{* * *} p \leq 0.001\right)$.

In concordance with previous studies (Cunningham et al., 2007; Michalovicz and Konat, 2014), the three ictogenic cytokines, IL-1 $\beta$, IL-6 and TNFa, surged in the blood following PIC challenge (Fig. 2). IL-6 $[F(6,20)=28.53, p<0.001]$ reached concentrations comparable to that of $C X C L 10$ at $6 \mathrm{~h}$, whereas $\operatorname{IL}-1 \beta[F(6,19)=5.69, \mathrm{p}=0.002]$ and $\operatorname{TNF\alpha }[\mathrm{F}(5,12)=8.14, \mathrm{p}<0.001]$ featured modest peaks. Like in the case of chemokines, blood concentration of these cytokines rapidly returned to the basal levels by 12 or $24 \mathrm{~h}$. Hippocampal level of IL-6 showed a slight, approximately 2-fold increase $6 \mathrm{~h}$ after $\mathrm{PIC}$ challenge $[\mathrm{F}(6,27)=4.83, \mathrm{p}<0.005]$, whereas, the levels of IL-1 $1 \beta[F(6,17)=0.90, p=0.516]$ and $\operatorname{TNF} \alpha[F(5,14)=2.05, p=0.134]$ were unchanged. 

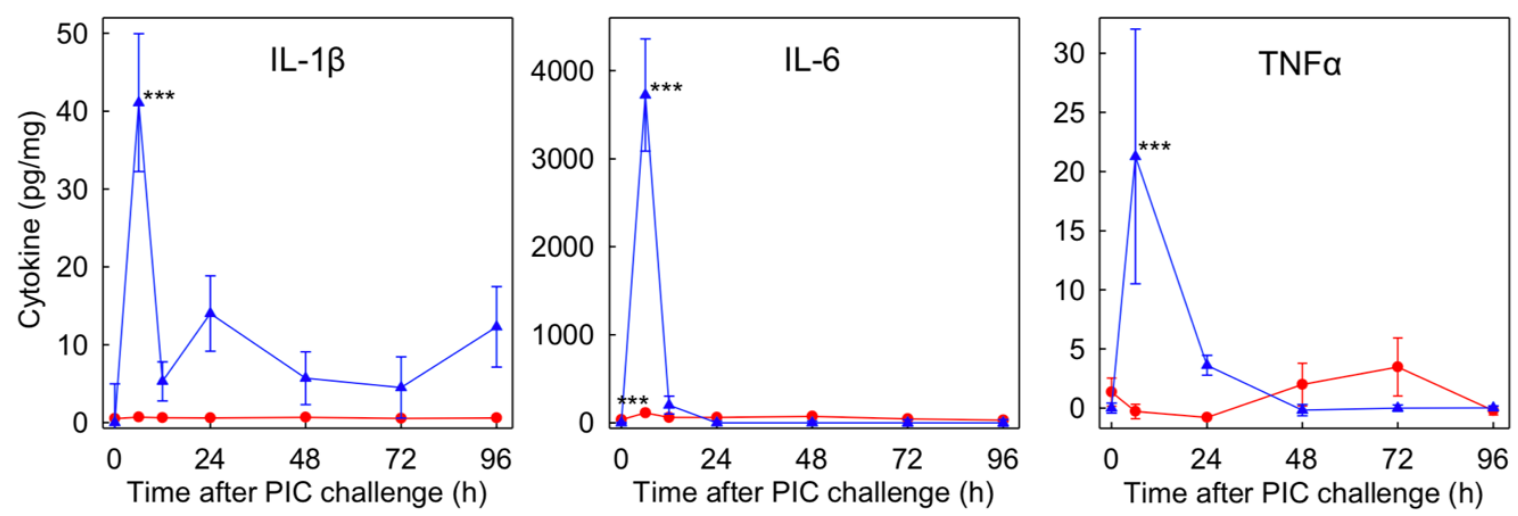

Figure 2. Production of IL-1 $\beta$, IL-6, and TNF $\alpha$ induced by peripheral PIC challenge. Mice were intraperitoneally injected with $12 \mathrm{mg} / \mathrm{kg}$ of PIC. Saline-injected mice served as respective controls. At different time points, blood (blue triangles) and hippocampal (red circles) levels of the cytokines were quantified by ELISA. The results are expressed as pg of chemokines per mg of total tissue protein. Symbols represent means \pm SEM from 4-7 animals. Asterisks denote significant differences from saline-injected controls $\left({ }^{* * *} p \leq 0.001\right)$.

Based on our previous study (Michalovicz et al., 2015b) showing that PIC challenge upregulates the expression of the complement system in the hippocampus, we profiled temporal changes in the levels of select complement proteins (Fig. 3). All proteins tested were present in the control hippocampus. C3 featured the highest basal level followed by C8b, C3a CfB (complement factor B), C5a and C9. C3 $[F(6,23)=5.32, p=0.001]$ and $C 8 b[F(6,33)=2.85, p=0.024]$ were transiently increased at 72 and $12 \mathrm{~h}$ post PIC, respectively. The level of C3a anaphylatoxin rapidly increased within the initial $24 \mathrm{~h}$ and approached a plateau at approximately $48 \mathrm{~h}$ after PIC challenge $[F(6,32)=6.88, p<0.001]$. $C 5 a$ anaphylatoxin displayed a more complex kinetics, as its levels increased rapidly at $12 \mathrm{~h}$, declined at $48 \mathrm{~h}$, and then increased again at 72-94 h [F(6, $32)=9.97, p<0.001] . C 9[F(6,32)=1.71, p=0.151]$ and $C f B[F(6,11)=3.07, p=0.051]$ were not affected by PIC challenge at any time point. 

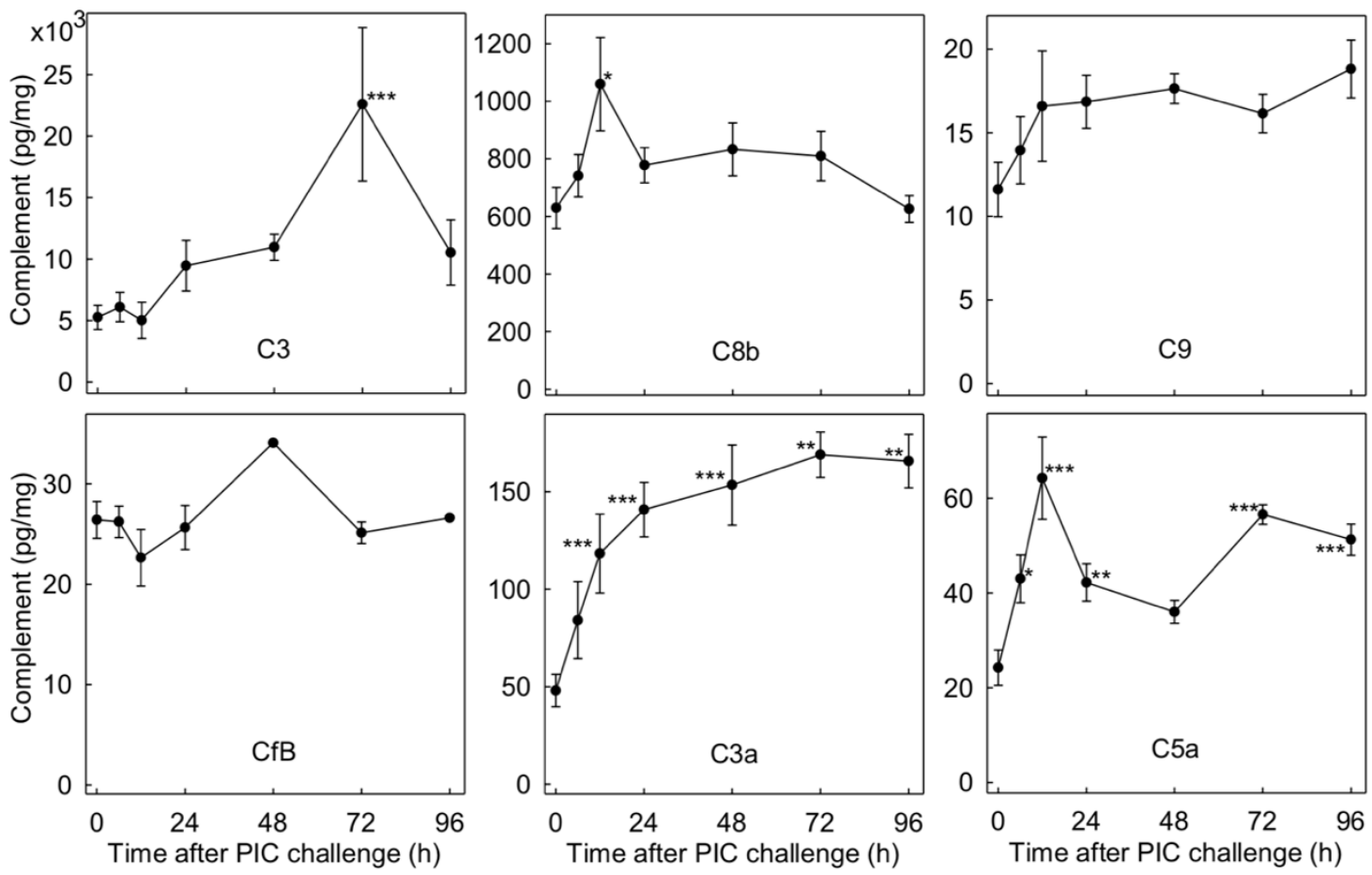

Figure 3. Hippocampal production of the complement proteins induced by peripheral PIC challenge. Mice were intraperitoneally injected with $12 \mathrm{mg} / \mathrm{kg}$ of PIC. Saline-injected mice served as respective controls. At different time points, hippocampal levels of the complement proteins were quantified by ELISA and expressed as pg of complement proteins per mg of total tissue protein. Symbols represent means \pm SEM from 4-7 animals. Asterisks denote significant differences from saline-injected controls $\left({ }^{*} p \leq 0.05 ;{ }^{* *} p \leq 0.01 ;{ }^{* * *} p \leq 0.001\right)$.

\subsection{DISCUSSION}

PIC has been commonly used to induce antiviral APR that features a surge in blood-borne cytokines including IFN $\beta$, IL-6, IL-12, TNF $\alpha$ and IL-1 $\beta$ (Pruett et al., 2004; Cunningham et al., 2007; Michalovicz and Konat, 2014). Herein, we found that PIC challenge also engenders an increase in circulating chemokines, i.e., CXCL10, CCL2, CXCL9, CCL7, CCL12, CXCL1 and CXCL2. Like the cytokines, the chemokines revealed an early, but transient elevation.

This blood cytokine/chemokine surge induces a robust generation of CXCL10, and to a lesser extent the generation of CXCL9, in the hippocampus. CXCL10 and CXCL9 are ligands of the common receptor, CXCR3. CXCL10 has been shown to modulate neuronal function in diverse in vitro systems. For example, in cell cultures, CXCL10 enhanced electrical activity of neurons (Nelson and Gruol, 2004), reduced the activity of GABAergic synapses and increased synaptic network activity (Cho et al., 2009). The exposure of hippocampal slices to exogenous CXCL10 
inhibited long-term potentiation (LTP) and altered presynaptic transmitter release through CXCR3 signaling (Vlkolinsky et al., 2004). Hippocampal levels of two ligands of CXCR2, CXCL1 and CXCL2, were also increased by PIC challenge. Signaling through CXCR2, the receptor for CXCL1 and CXCL2, has also been shown to increase neuronal excitability (Ragozzino et al., 1998; Lax et al., 2002; Wang et al., 2008). Consequently, hippocampal elevation of the four chemokines found in the present study (Fig. 1) strongly implicates them as candidate molecules that mediate the development of hyperexcitability, and thus, seizure hypersusceptibility instigated by peripheral PIC challenge. However, the combined maximal concentration of CXCL9 and CXCL10 was approximately 9-fold higher than the combined maximal concentration of CXCL1 and CXCL2. Consequently, CXCR3 activation is most likely the primary signaling pathway.

In addition to the direct effects on neuronal excitability discussed above, chemokines may induce hyperexcitability through alternative mechanisms. For example, CXCL10 is a potent activator and chemoattractant of microglia (Rappert et al., 2004; Clarner et al., 2015), and microglia are well known for their ability to disassemble neuronal synapses (Ji et al., 2013; Kettenmann et al., 2013). Therefore, activated microglia may displace presynaptic terminals from GABAergic synapses leading to disinhibition, and thus, hyperexcitability and seizure hypersusceptibility. In support of such a mechanism, peripheral challenge with a bacterial mimetic, lipopolysaccharide (LPS) has been shown to induce hyperexcitability of cortical neurons through microglia-mediated displacement of presynaptic GABAergic terminals (Chen et al., 2014).

IL-1 $\beta, I L-6$, and TNFa have been shown to be ictogenic in a variety of experimental systems (Li et al., 2011). However, in spite of the significant increase in their blood concentrations instigated by PIC challenge, only IL-6 was found minimally ( 2-fold) increased over basal level in the hippocampus (Fig. 2). This is in contrast to the robust elevation of the chemokines (Fig. 1). Therefore, IL-6, IL-1 $\beta$ and TNF $\alpha$ are not likely to be involved in the induction of hyperexcitable phenotype following PIC challenge.

The complement system has been implicated in the modulation of neuronal network function through synaptic scaling (Schafer et al., 2012). Previously, we reported the upregulation of hippocampal mRNA encoding several complement components following PIC challenge (Michalovicz et al., 2015b). In concordance with the increased abundance of the C3 mRNA, we 
found the elevation of C3 protein (Fig. 3). However, while the mRNA peaked at $24 \mathrm{~h}, \mathrm{C} 3$ protein peaked at $72 \mathrm{~h}$ indicating the possibility of delayed translation of the message. Also, there was no upregulation of the $C 8$ gene expression of the message level, but a significant upregulation at the protein level was evident at $12 \mathrm{~h}$ post PIC. This discrepancy indicates a posttranscriptional regulation of these genes. The most notable is the divergence in the expression of the Cfb gene. The Cfb message peaked to 53-fold over control $24 \mathrm{~h}$ after PIC challenge (Michalovicz et al., 2015b). Also, an increase in the CfB protein was evident in the tissue by immunofluorescence. However, no significant elevation of hippocampal CfB was detected by ELISA (Fig. 3). CfB is a single chain polypeptide that upon activation is proteolytically cleaved to $\mathrm{Ba}$ and $\mathrm{Bb}$ fragments. $\mathrm{Bb}$ binds to membrane-linked $\mathrm{C} 3 \mathrm{~b}$ subunit, and subsequently to $\mathrm{C} 5$ to form the alternative pathway convertase $\mathrm{C} 3$ and convertase $\mathrm{C} 5$, respectively. It can be envisaged that $\mathrm{PIC}$ challenge increases the synthesis of CfB that is rapidly activated by cleavage. Therefore, the cleavage of the CfB polypeptide could abolish antibody binding in ELISA, but the fragments were still detected by the antibody used for immunofluorescence.

The generation of anaphylatoxins, C3a and C5a, the products of C3 and C5 convertases, substantiates the activation of the complement cascades in the hippocampus. Interestingly, the elevated concentration of C3a and C5a did not dropped even $96 \mathrm{~h}$ after PIC challenge (Fig. 3). This kinetics is in contrast with the chemokines that dwindle to the control level by $48 \mathrm{~h}$, or sooner. Also, seizure hypersusceptibility decreases gradually after $24 \mathrm{~h}$ and ultimately disappears $96 \mathrm{~h}$ after PIC challenge (Michalovicz and Konat, 2014). Consequently, the anaphylatoxins are not likely to mediate the increase in seizure susceptibility.

In summary, the generation of CXCL10 in the hippocampus induced by peripheral PIC challenge is likely the primary molecular event that drives the development of neuronal hyperexcitability and ensuing seizure hypersusceptibility.

\section{Acknowledgements}

This study was supported by WVU Senate and WVU PSCOR grants. The content is solely the responsibility of the authors and does not necessarily represent the official views of the funding agencies.

\section{Conflict of interest}

The authors declare no conflicts of interest. 


\subsection{REFERNECES}

Ben-Ari Y, Cossart R (2000) Kainate, a double agent that generates seizures: two decades of progress. Trends Neurosci 23:580-587.

Chen Z, Jalabi W, Hu W, Park HJ, Gale JT, Kidd GJ, Bernatowicz R, Gossman ZC, Chen JT, Dutta R, Trapp BD (2014) Microglial displacement of inhibitory synapses provides neuroprotection in the adult brain. Nat Commun 5:4486.

Cho J, Nelson TE, Bajova H, Gruol DL (2009) Chronic CXCL10 alters neuronal properties in rat hippocampal culture. J Neuroimmunol 207:92-100.

Clarner T, Janssen K, Nellessen L, Stangel M, Skripuletz T, Krauspe B, Hess FM, Denecke B, Beutner C, Linnartz-Gerlach B, Neumann H, Vallieres L, Amor S, Ohl K, Tenbrock K, Beyer C, Kipp M (2015) CXCL10 triggers early microglial activation in the cuprizone model. J Immunol 194:3400-3413.

Cunningham C, Campion S, Teeling J, Felton L, Perry VH (2007) The sickness behaviour and CNS inflammatory mediator profile induced by systemic challenge of mice with synthetic double-stranded RNA (poly I:C). Brain Behav Immun 21:490-502.

Dantzer R (2006) Cytokine, sickness behavior, and depression. Neurol Clin 24:441-460.

Dantzer R, Kelley KW (2007) Twenty years of research on cytokine-induced sickness behavior. Brain Behav Immun 21:153-160.

Hunsberger HC, Konat GW, Reed MN (2017) Peripheral viral challenge elevates extracellular glutamate in the hippocampus leading to seizure hypersusceptibility. J Neurochem 141:341-346.

Hunsberger HC, Wang D, Petrisko TJ, Alhowail A, Setti SE, Suppiramaniam V, Konat GW, Reed MN (2016) Peripherally restricted viral challenge elevates extracellular glutamate and enhances synaptic transmission in the hippocampus. J Neurochem 138:307-316.

Ji K, Miyauchi J, Tsirka SE (2013) Microglia: an active player in the regulation of synaptic activity. Neural Plast 2013:627325.

Kettenmann H, Kirchhoff F, Verkhratsky A (2013) Microglia: new roles for the synaptic stripper. Neuron 77:10-18.

Kirschman LT, Borysiewicz E, Fil D, Konat GW (2011) Peripheral immune challenge with dsRNA enhances kainic acid-induced status epilepticus. Metab Brain Dis 26:91-93.

Lax P, Limatola C, Fucile S, Trettel F, Di BS, Renzi M, Ragozzino D, Eusebi F (2002) Chemokine receptor CXCR2 regulates the functional properties of AMPA-type glutamate receptor GluR1 in HEK cells. J Neuroimmunol 129:66-73.

Li G, Bauer S, Nowak M, Norwood B, Tackenberg B, Rosenow F, Knake S, Oertel WH, Hamer HM (2011) Cytokines and epilepsy. Seizure 20:249-256.

Michalovicz LT, Konat GW (2014) Peripherally restricted acute phase response to a viral mimic alters hippocampal gene expression. Metab Brain Dis 29:75-86.

Michalovicz LT, Lally BE, Konat GW (2015) Peripheral challenge with a viral mimic upregulates expression of the complement genes in the hippocampus. J Neuroimmunol 285:137142.

Nelson TE, Gruol DL (2004) The chemokine CXCL10 modulates excitatory activity and intracellular calcium signaling in cultured hippocampal neurons. J Neuroimmunol 156:7487.

Pruett SB, Schwab C, Zheng Q, Fan R (2004) Suppression of innate immunity by acute ethanol administration: a global perspective and a new mechanism beginning with inhibition of signaling through TLR3. J Immunol 173:2715-2724.

Quan N, Banks WA (2007) Brain-immune communication pathways. Brain Behav Immun 21:727-735. 
Ragozzino D, Giovannelli A, Mileo AM, Limatola C, Santoni A, Eusebi F (1998) Modulation of the neurotransmitter release in rat cerebellar neurons by GRO beta. Neuroreport 9:36013606.

Rappert A, Bechmann I, Pivneva T, Mahlo J, Biber K, Nolte C, Kovac AD, Gerard C, Boddeke HW, Nitsch R, Kettenmann H (2004) CXCR3-dependent microglial recruitment is essential for dendrite loss after brain lesion. J Neurosci 24:8500-8509.

Schafer DP, Lehrman EK, Kautzman AG, Koyama R, Mardinly AR, Yamasaki R, Ransohoff RM, Greenberg ME, Barres BA, Stevens B (2012) Microglia sculpt postnatal neural circuits in an activity and complement-dependent manner. Neuron 74:691-705.

Scheid R, Teich N (2007) Neurologic manifestations of ulcerative colitis. Eur J Neurol 14:483493.

Tellez-Zenteno JF, Matijevic S, Wiebe S (2005) Somatic comorbidity of epilepsy in the general population in Canada. Epilepsia 46:1955-1962.

Verrotti A, Tocco AM, Coppola GG, Altobelli E, Chiarelli F (2009) Afebrile benign convulsions with mild gastroenteritis: a new entity? Acta Neurol Scand 120:73-79.

Vlkolinsky R, Siggins GR, Campbell IL, Krucker T (2004) Acute exposure to CXC chemokine ligand 10, but not its chronic astroglial production, alters synaptic plasticity in mouse hippocampal slices. J Neuroimmunol 150:37-47.

Wang JG, Strong JA, Xie W, Yang RH, Coyle DE, Wick DM, Dorsey ED, Zhang JM (2008) The chemokine CXCL1/growth related oncogene increases sodium currents and neuronal excitability in small diameter sensory neurons. Mol Pain 4:38. 


\title{
CHAPTER 3: NEURONAL CXCL10/CXCR3 AXIS MEDIATES THE INDUCTION OF CEREBRAL HYPEREXCITABILITY BY PERIPHERAL VIRAL CHALLENGE
}

Original Article: In Revision at Frontiers in Neuroscience at time of submission

\author{
Petrisko T. J. ${ }^{1 \#}$, Bloemer J. ${ }^{2 \#}$, Srinivas S. ${ }^{2}$, Pinky P. ${ }^{2}$, Du Y. ${ }^{2}$, Setti S. ${ }^{2}$, Hong, H. ${ }^{3,4}$, \\ Suppiramaniam V. ${ }^{2,5}$, Konat G.W. ${ }^{1 *}$, Reed M.N. ${ }^{2,5 *}$
}

\begin{abstract}
${ }^{1}$ Departments of Biochemistry Neuroscience, West Virginia University School of Medicine, Morgantown, WV, USA

${ }^{2}$ Drug Discovery and Development, School of Pharmacy, Auburn University, Auburn, AL, USA

${ }^{3}$ Department of Pharmacy, the First Affiliated Hospital of Xiamen University, Xiamen, Fujian, China

${ }^{4}$ Key Laboratory of Neuropsychiatric Diseases, Jiangsu Key Laboratory of Drug Discovery for Metabolic Diseases, and State Key Laboratory of Natural Medicines, China Pharmaceutical University, Nanjing, Jiangsu, China ${ }^{5}$ Center for Neuroscience Initiative, Auburn University, Auburn, AL, USA

\#These two authors contributed equally to this work.
\end{abstract}

${ }^{*}$ These co-senior authors contributed equally to the conception and experimental design of the projects as well as to the interpretation of results. 


\subsection{ABSTRACT}

Peripheral infections can potently exacerbate neuropathological conditions, though the underlying mechanisms are poorly understood. We have previously demonstrated that intraperitoneal (i.p.) injection of a viral mimetic, polyinosinic-polycytidylic acid (PIC) induces a robust generation of CXCL10 chemokine in the hippocampus. The hippocampus also features hyperexcitability of neuronal circuits following PIC challenge. The present study was undertaken to determine the role of CXCL10 in mediating the development of hyperexcitability in response to PIC challenge. Briefly, young female C57BL/6 mice were i.p. injected with PIC, and after 24 $\mathrm{h}$, the brains were analyzed by confocal microscopy. CXCL10 staining of neuronal perikarya and a less intense staining of the neuropil was observed in the hippocampus and cortex. CXCL10 staining was also evident in a subpopulation of astrocytes, whereas microglia were CXCL10 negative. CXCR3, the cognate receptor of CXCL10 was present exclusively on neurons, indicating that the CXCL10/CXCR3 axis operates through an autocrine/paracrine neuronal signaling. Blocking cerebral CXCR3 through intracerebroventricular injection of a specific inhibitor, AMG487, abrogated PIC challenge-induced increase in basal synaptic transmission and long-term potentiation (LTP), as well as the reduction of paired-pulse facilitation (PPF). The PIC-mediated abolishment of long-term depression (LTD) was also restored after application of AMG487. Moreover, CXCR3 inhibition attenuated seizure hypersensitivity induced by PIC challenge. The efficacy of AMG487 strongly strengthens the notion that CXCL10/CXCR3 axis mediates the induction of cerebral hyperexcitability by PIC challenge.

\subsection{INTRODUCTION}

Peripheral infections are important comorbid factors for the major neuropathological conditions. For example, peripheral infections exacerbate dementia in Alzheimer's disease (AD) (Murray et al., 1993; George et al., 1997; Nee and Lippa, 1999; Holmes et al., 2003; Holmes, 2013), relapses in multiple sclerosis (MS) (Andersen et al., 1993; Edwards et al., 1998; Buljevac et al., 2002; Libbey and Fujinami, 2010) and seizures (Tellez-Zenteno et al., 2005; Scheid and Teich, 2007; Verrotti et al., 2009). It is generally believed that inflammatory agents generated during the initial innate immune response to the invading microbes, i.e., the acute phase response (APR), are relayed to the brain, and by augmenting the ongoing neuropathology exacerbate disease symptoms. However, the underlying cellular/molecular mechanisms have not been defined. 
We have developed a preclinical murine model to study mechanisms by which APR exerts its effects on the brain. In this model, APR is induced by intraperitoneal injection of the epitomic viral mimetic, polyinosinic-polycytidylic acid (PIC). We have demonstrated that PIC challenge induces hyperexcitability of neuronal networks as seen from a profound increase in the basal synaptic transmission and long term potentiation (LTP) in hippocampal slices (Hunsberger et al., 2016), as well as from hypersusceptibility to kainic acid (KA)-induced status epilepticus (Kirschman et al., 2011; Michalovicz and Konat, 2014; Hunsberger et al., 2017). Because neuronal hyperexcitability is an invariable feature of the major neuropathologies (Esclapez et al., 1999; Lehmann et al., 2000; Buljevac et al., 2002; Holmes et al., 2003; Caramia et al., 2004; Palasik et al., 2005; Tellez-Zenteno et al., 2005; Scheid and Teich, 2007; Verrotti et al., 2009; Khedr et al., 2011; Penzes et al., 2011; Rossi et al., 2012; Scharfman, 2012; Huynh et al., 2013; Yener and Basar, 2013; Eikermann-Haerter, 2014), it might provide a mechanistic link for the exacerbating effects of peripheral inflammation on disease progression.

At the molecular level, PIC challenge induces a fulminant but transient increase of several inflammatory cytokines (IFN $\beta$, IL-6, IL1 $\beta$ and TNF $\alpha$ ) and chemokines (CXCL10, CCL2, CXCL9, CCL7 and CCL12) in the blood (Michalovicz and Konat, 2014; Petrisko and Konat, 2017). This "cytokine storm" in turn, leads to a robust generation of CXCL10 in the hippocampus, whereas other major inflammatory mediators are either only slightly elevated (CXCL1, CXCL2, CXCL9, IL-6), or unchanged (IL-1 $\beta, T N F \alpha$ ). The expression of the Cxc/10 mRNA is also massively upregulated in the hippocampus following PIC challenge (Michalovicz and Konat, 2014), indicating that CXCL10 is produced in situ in the brain.

Because CXCL10 is a potent modulator of neuronal activity (Nelson and Gruol, 2004; VIkolinsky et al., 2004; Cho et al., 2009), it seems plausible that it might be a putative molecule, which acting through its cognate receptor, CXCR3, drives the development of hyperexcitability. This is congruent with the emerging role of CXCL10 as an important player in diverse neuroinflammatory and neurodegenerative diseases (Michlmayr and McKimmie, 2014b).

The present study was undertaken to identify cellular origin of cerebral CXCL10 production instigated by PIC challenge. We also appraised the cellular origin of CXCR3, and its involvement in the development of neuronal hyperexcitability. 


\subsection{MATERIALS AND METHODS}

\subsubsection{ANIMALS}

Eight-week old female C57BL/6J mice obtained from Charles River Laboratories (Wilmington, MA) were housed with free access to food and water in a humidity- and temperature-controlled rooms under a 12:12 h light-dark cycle. Mice in all experimental groups were matched by weight prior to treatments. All experimental procedures were approved by the West Virginia University and Auburn University Animal Care and Use Committee and conducted in compliance with the guidelines published in the NIH Guide for the Care and Use of Laboratory Animals.

\subsubsection{DRUG ADMINISTRATION}

Peripheral APR was induced by a single i.p. injection of $12 \mathrm{mg} / \mathrm{kg}$ of ultrapure PIC (Invivogen, San Diego, CA) in $100 \mu \mathrm{L}$ of saline. Mice injected with equivolume saline served as controls. To verify successful PIC injection, the development of sickness behavior was assessed after $6 \mathrm{~h}$ by the rearing test (Michalovicz and Konat, 2014).

To block CXCR3, a specific inhibitor, AMG-487 (Tocris), was administered by intracerebroventricular (i.c.v.) injection. Briefly, mice were anesthetized with isoflurane (1.9\%$3.4 \%$ inhalation; continuous) and immobilized on a stereotaxic frame (Kopf, Tujunga CA). Three $\mathrm{mg} / \mathrm{kg}$ of AMG-487 (Tocris, Bristol, UK) in $5 \mu \mathrm{L}$ of artificial cerebrospinal fluid containing $20 \%$ of DMSO (ACSF) was delivered gradually $(0.5 \mu \mathrm{l} / \mathrm{min})$ into the cerebral ventricles through a $26 \mathrm{~s}-$ gauge needle as a bilateral injection of $2.5 \mathrm{uL}$ per side. The coordinates from bregma were: anteroposterior: $-0.45 \mathrm{~mm}$, mediolateral: $+/-0.95 \mathrm{~mm}$, and dorsoventral: $-2.6 \mathrm{~mm}$ (Paxinos and Franklin 2001). The needle was left in place for $5 \mathrm{~min}$ to minimize back-flux of the injectate. After $2 \mathrm{~h}$, the animals were PIC-challenged as above.

\subsubsection{INDUCTION OF SEIZURES}

Twenty-four hours after PIC injection, seizures were induced via subcutaneous (s.c.) injection of $12 \mathrm{mg} / \mathrm{kg}$ of kainic acid (KA; Sigma Chemical Co., St Louis, MO), and seizure severity was graded as previously described (Kirschman et al., 2011; Hunsberger et al., 2017) using the 6step scale (Morrison et al., 1996). Seizure cessation was defined as two consecutive seizure severity scores of 0 . Cumulative seizure scores were calculated as the summation of all scores over the entire period of status epilepticus. 


\subsubsection{EXTRACELLULAR FIELD POTENTIAL RECORDING}

Twenty-four hours after PIC injection, animals were euthanized with carbon dioxide, and 350$\mu \mathrm{m}$ thick coronal slices through the dorsal hippocampus were prepared using a Leica VT1200S Vibratome (Leica Microsystems, Wetzlar, Germany). Electrophysiological measurements, i.e., basal synaptic transmission, paired-pulse facilitation (PPF), long-term potentiation (LTP) and long-term depression (LTD), were performed as previously described (Parameshwaran et al., 2013; Bhattacharya et al., 2015; Hunsberger et al., 2016; Bhattacharya et al., 2017).

\subsubsection{CONFOCAL MICROSCOPY}

$24 \mathrm{~h}$ after PIC challenge, mice were deeply anesthetized by i.p. injection of $65 \mathrm{mg} / \mathrm{kg}$ of pentobarbital (Beauthanasia, Patterson Veterinary, Devens, MA), sacrificed by pneumothorax, and transaortically perfused with saline followed by $4 \%$ paraformaldehyde. The brains were dissected, cryoprotected, and cut into $30 \mu \mathrm{m}$ coronal sections using the ThermoFisher Scientific HM450 Sliding Microtome (Thermo Fisher Scientific, Waltham, MA USA). Free-floating immunofluorescent staining of CXCL10, GFAP and lba1 was performed as previously described (Michalovicz et al., 2015b). For CXCR3 localization, Triton X-100 concentration was increased to $0.4 \%$. Additionally, for microglia co-staining, sections were incubated with anti-Cd11b antibody for $96 \mathrm{~h}$ at $4^{\circ} \mathrm{C}$ with rat-anti-CXCR3 being added for the last $12 \mathrm{~h}$. Sections were mounted to slides with Prolong Gold (Thermo Fisher Scientific, Waltham, MA USA), and imaged using the Nikon A1R Confocal microscope (Nikon Instruments, Melville, NY). Z-stacks were taken with a 60X objective every $0.125 \mu \mathrm{m}$, and 3D projections were rendered using NIS Elements Advanced Research imaging software (Nikon Instruments, Melville, NY). For the colocalization of CXCL10 the primary antibodies were: goat-anti-CXCL10 (R\&D Systems, Minneapolis, MN), mouse-anti-NeuN (Millipore, Burlington, MA), rabbit-anti-GFAP (Dako, Santa Clara, CA) and rabbit-anti-lba1 (Wako Chemicals, Richmond, VA). Secondary antibodies were: anti-goat conjugated with Alexa Fluor 555, and anti-mouse and anti-rabbit conjugated with Alexa Fluor 488 (Thermo Fisher Scientific, Waltham, MA USA). For the co-localization of CXCR3 the primary antibodies were: rabbit-anti-CXCR3 (Novus Biologicals, Centennial, CO), mouse-antiNeuN (Millipore, Burlington, MA), mouse-anti-GFAP (Millipore, Burlington, MA) and rat-antiCD11b conjugated to BD Horizon BV480 (BD Biosciences, Franklin Lakes, NJ). Secondary antibodies were: anti-rabbit conjugated with Alexa Fluor 555, and anti-mouse conjugated with Alexa Fluor 555 (Thermo Fisher Scientific, Waltham, MA USA). 


\subsubsection{STATISTICAL ANALYSIS}

The data were analyzed by ANOVA, expressed as means \pm SEM. Statistical comparisons between groups were performed using Tukey post hoc tests. Differences between groups were considered significant at $P<0.05$.

\subsection{RESULTS}

Immunofluorescent analysis performed $24 \mathrm{~h}$ after PIC challenge revealed intense CXCL10 staining throughout the brain, whereas a negligible staining was observed in the brain of salineinjected mice. Representative confocal images of the CA1 hippocampal region and the motor cortex are shown in the upper panels of Fig. 1. Most of CXCL10 staining was localized to the cytoplasm of neuronal perikarya as revealed by neuron-specific NeuN antibody (Fig. 1, lower panels). The staining extended into the proximal parts of neuronal processes. However, the intensity of CXCL10 staining among NeuN-positive neurons varied. This variation was particularly well demonstrable in the cortex where individual neurons were better separated. Also, a subpopulation of astrocytes expressed CXCL10. The staining was restricted to the cell bodies. In contrast, no CXCL10 staining co-localized with Iba1-positive microglia. The same cell-specific co-localization of CXCL10 was observed at shorter time intervals after PIC challenge, i.e., 6 and $12 \mathrm{~h}$ (not shown). 

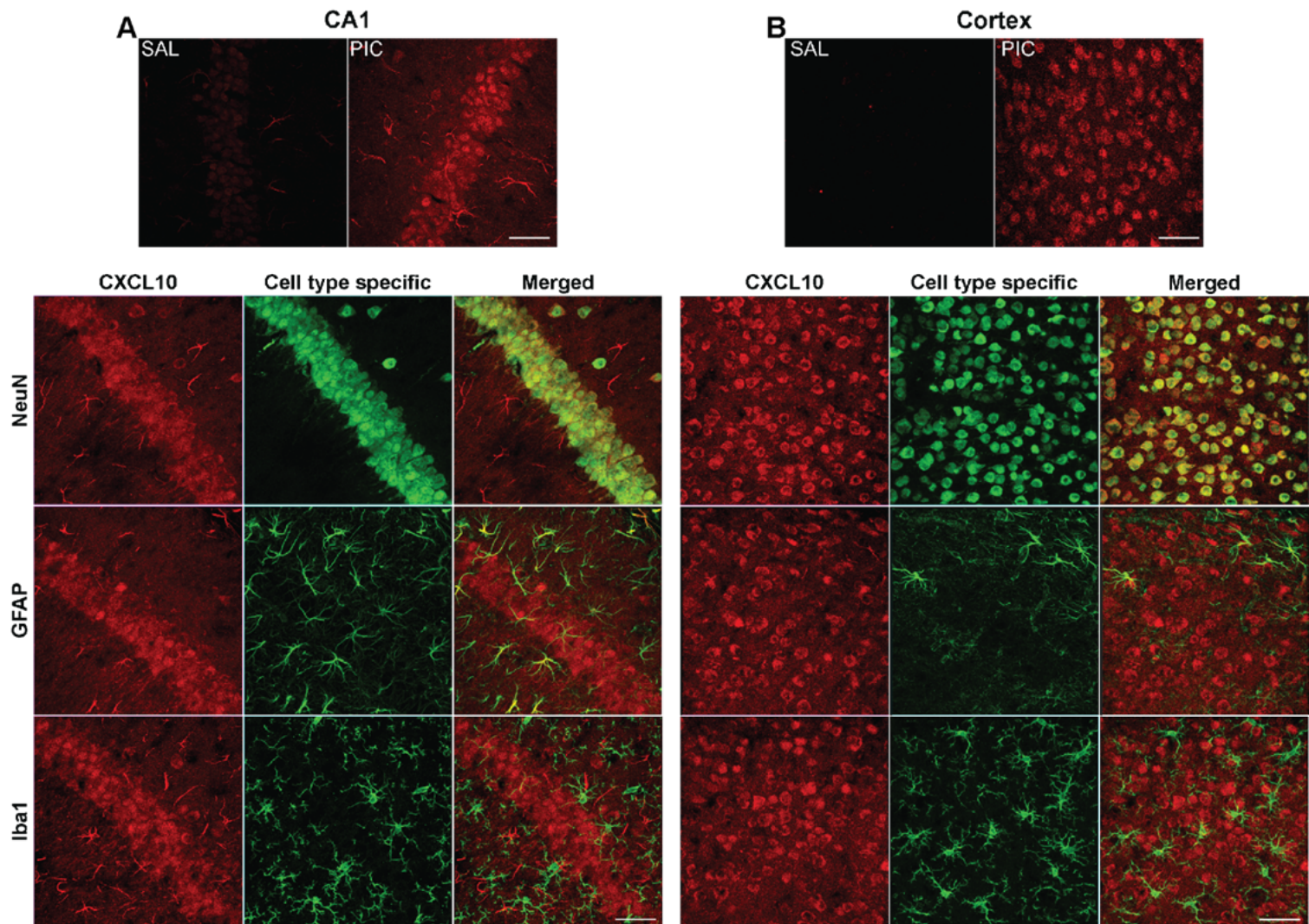

Figure 1. Cellular origin of cerebral CXCL10 production following PIC challenge. Mice were i.p. injected with $12 \mathrm{mg} / \mathrm{kg}$ of PIC or saline (SAL), and after $24 \mathrm{~h}$, analyzed by immunohistochemistry. Upper panels The expression of CXCL10 (red) in the hippocampal CA1 region (A) and in the cortex (B). Lower panels Cell-specific expression of CXCL10 (red) in the CA1 and in the cortex of PIC-challenged mice. Anti-NeuN, anti-GFAP and anti-Iba1 antibodies (green) were used to identify neurons, astrocytes and microglia, respectively. Confocal images were captured at 60X magnification. Scale bars represent $50 \mu \mathrm{m}$. 
As depicted in Fig. 2, neurons also expressed the cognate receptor of CXCL10, CXCR3. In congruence with the previously observed lack of upregulation of the Cxcr3 mRNA expression by PIC challenge (Fil et al., 2011), there was no detectable difference in CXCR3 staining intensity between brain tissue from PIC-injected and saline injected mice (upper panels). The expression was confined to the cell surface of neuronal perikarya. Although, CXCR3 staining was also evident in neuronal processes, particularly, in the cortex. CXCR3 expression was evident throughout the brain. No apparent CXCR3 staining was detectable in either astrocytes or microglia.
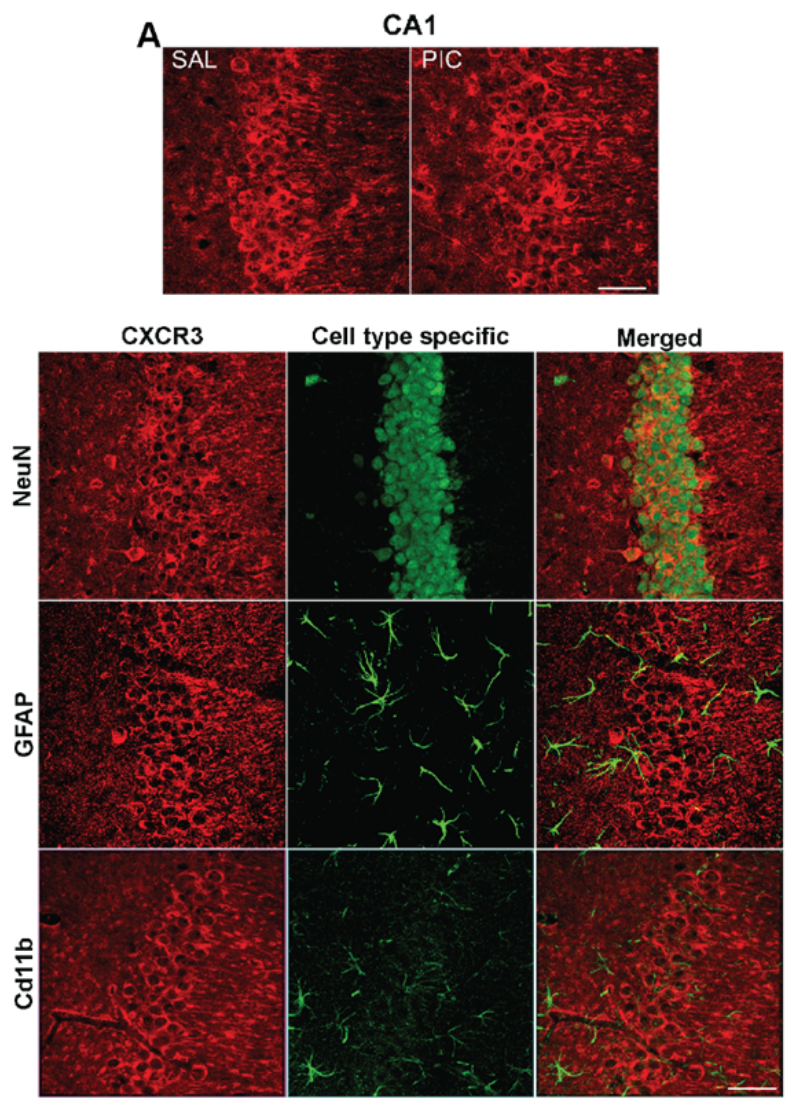

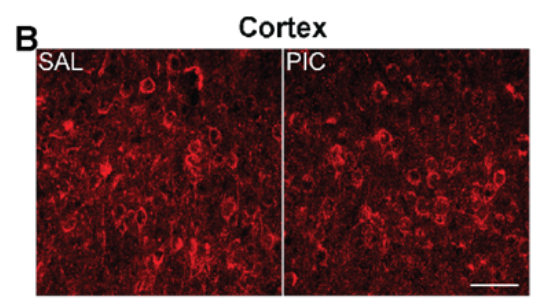

CXCR3

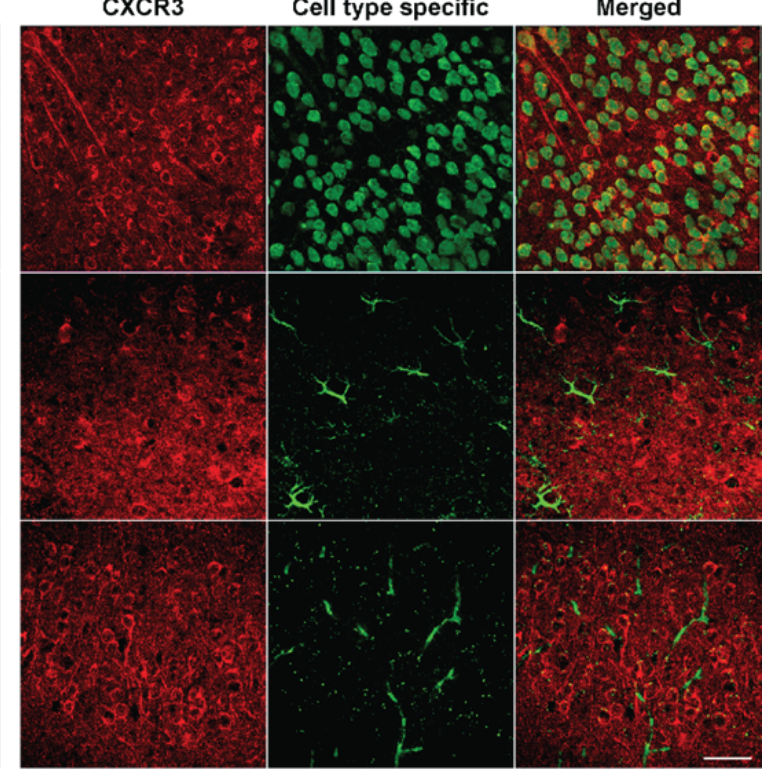

Figure 2. Cellular expression of CXCR3. Mice were i.p. injected with $12 \mathrm{mg} / \mathrm{kg}$ of PIC or saline (SAL), and after $24 \mathrm{~h}$, analyzed by immunohistochemistry. Upper panels The expression of CXCR3 (red) in the hippocampal CA1 region (A) and in the cortex (B). Lower panels; cellspecific expression of CXCR3 (red) in the CA1 and in the cortex of PIC-challenged mice. AntiNeuN, anti-GFAP and anti-CD11b antibodies (green) were used to identify neurons, astrocytes and microglia, respectively. Confocal images were captured at 60X magnification. Scale bars represent $50 \mu \mathrm{m}$. 
To assess the role of the CXCL10/CXCR3 axis in the induction of neuronal hyperexcitability, we used i.c.v. injection of a specific inhibitor, AMG487, to block CXCR3 signaling before challenging the animals with PIC. To determine whether the inhibitor alters the PIC-induced sickness behavior, we measured the loss of body weight $24 \mathrm{~h}$ after PIC challenge. As previously observed (Cunningham et al., 2007), the injection of saline and PIC induced approximately 1.5 and $5.9 \%$ body loss as assessed $24 \mathrm{~h}$ later (Fig. 3). No additional increase in the weight loss was found in mice injected with PIC and AMG487.
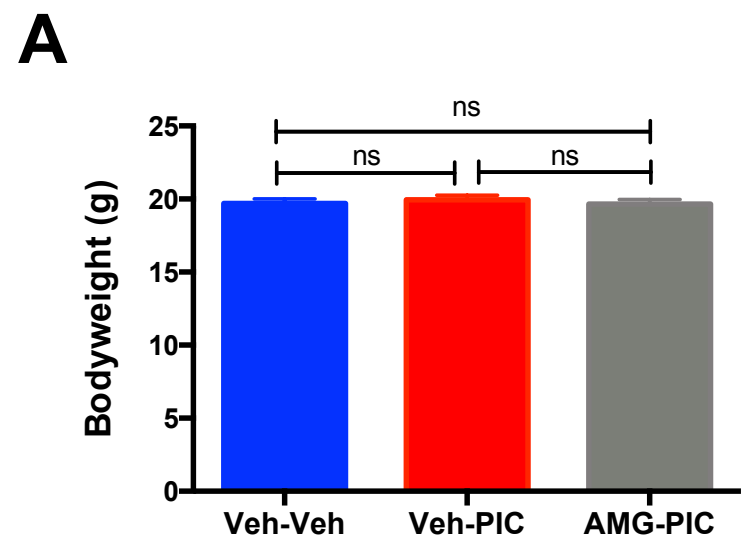
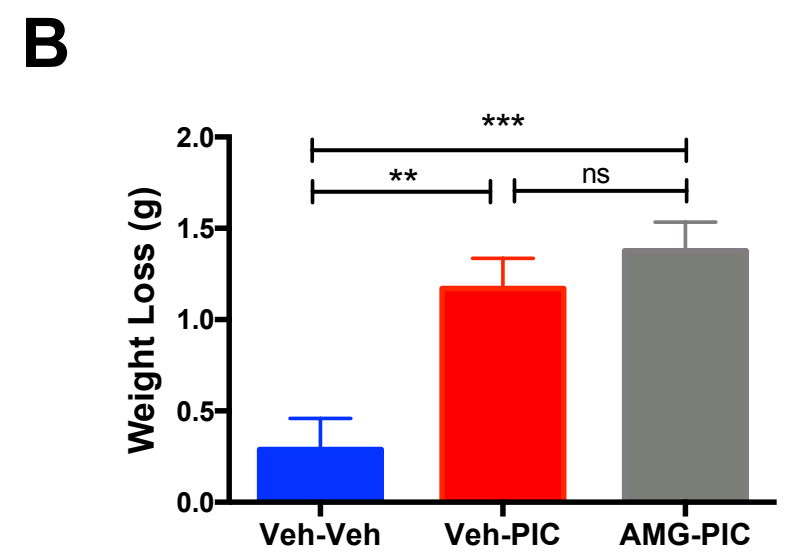

Figure 3. Weight loss in PIC challenged mice. Mice received a bolus of i.c.v. injection of AMG487 (3 mg/kg) or vehicle (VEH). Two hours later, mice were weighed prior to induction of the acute antiviral response via an i.p. injection of $12 \mathrm{mg} / \mathrm{kg}$ of PIC in saline (a). Mice injected with $100 \mu \mathrm{L}$ of saline served as vehicle controls. Twenty-four hours after PIC injection, mice were weighed again, and the amount of weight lost was determined (b). Bars represent means \pm SEM from 5 to 6 mice per group. (ANOVA followed by Tukey's multiple comparisons test; ${ }^{* *} p$ $\leq 0.01,{ }^{* * *} p \leq 0.001$; ns - not significantly different).

Basal synaptic transmission was evaluated in hippocampal slices $24 \mathrm{~h}$ after PIC challenge (Fig. 4). At stimulus intensities from 40 to $100 \mu \mathrm{A}$, basal synaptic transmission increased by approximately $85 \%$ in the slices from PIC-challenged compared to vehicle-injected mice.

CXCR3 blockade abrogated this increase (Fig. 4A). To determine whether there is a relationship between pre- and post- synaptic responses, fiber volley, which represents the presynaptic action potential in response to stimulus, was compared to postsynaptic responses represented as field excitatory postsynaptic potentials (fEPSPs) using linear regression analysis. PIC challenged mice exhibited increased postsynaptic responses to the same fiber volley amplitude compared to the vehicle-treated mice, whereas blockade of CXCR3 mitigated this increase (Fig. 4B). To determine if the hyperexcitability of the neurons following PIC challenge are due to presynaptic modifications, the probability of neurotransmitter release was examined by 
measuring paired pulse facilitation (PPF). There was a significant reduction in PPF at the short stimulus intervals in slices from PIC-challenged vs. vehicle-injected mice (Fig. 4C), indicating an increase in pre-synaptic release probability. The drop in $\mathrm{EPSP}_{2} / \mathrm{EPSP}_{1}$ was 35 and $25 \%$ at 25 and 50 ms stimulus intervals, respectively. No significant changes were evident at longer stimulus intervals. The PIC challenge-induced decrease in PPF was attenuated by the pretreatment of mice with AMG487. To test if the changes in PPF in PIC-challenged mice are due to presynaptic axonal recruitment, fiber volley amplitude versus stimulus intensities was compared. PIC challenge had no effect on the fiber volley amplitude across various stimulus intensities (Fig. 4D), which indicates no changes in presynaptic axon recruitment.

A

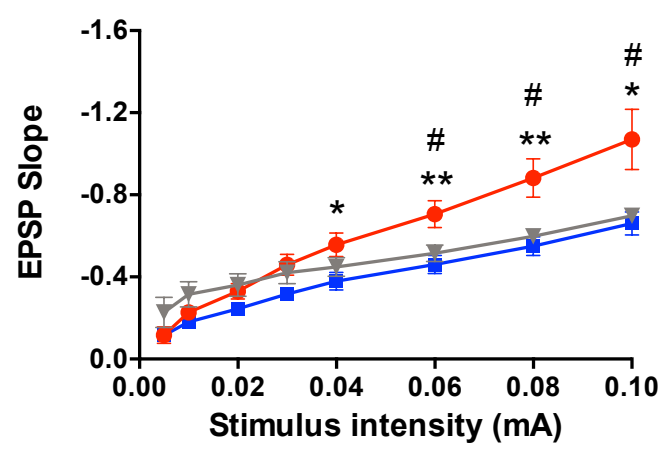

C

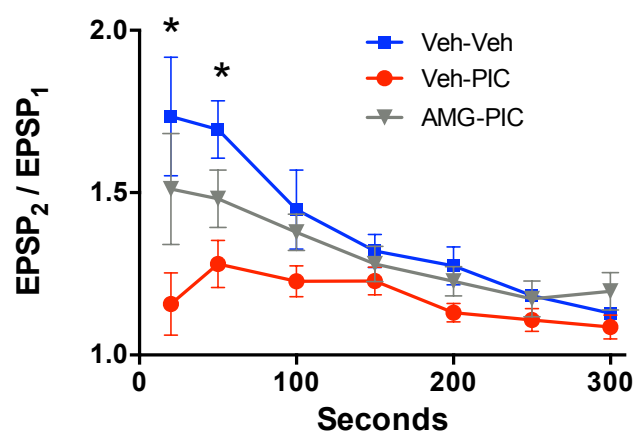

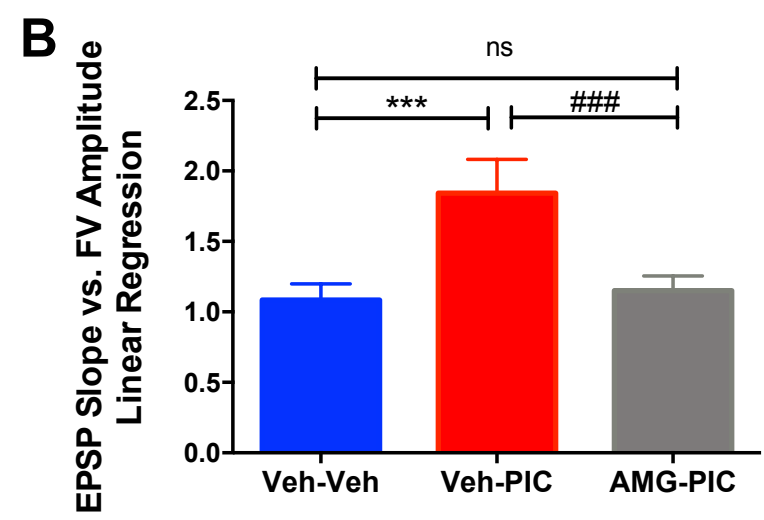

D

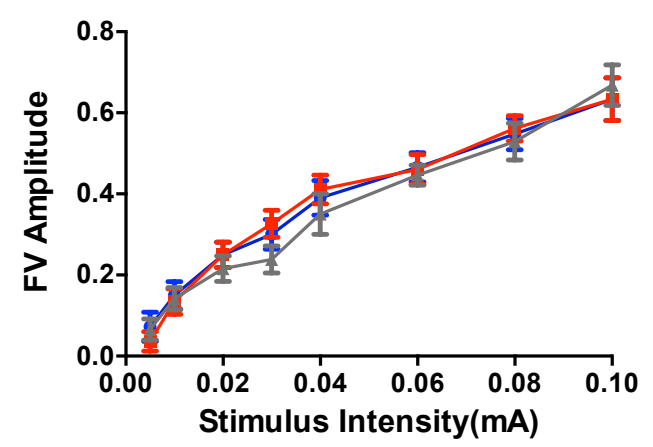

Figure 4. Synaptic transmission in hippocampal slices. (A) Basal synaptic transmission represented by the fEPSP slope measured at increasing stimulus intensities. (B) Fiber volley (FV) analysis represented by the FV amplitude measured at increasing stimulus intensities. (C) Paired-pulse facilitation expressed as the change of ratio of the second stimulus fEPSP to the first stimulus fEPSP slope plotted as a function of interstimulus interval. (D) Basal synaptic transmission represented by the slope of the linear regression between $\mathrm{AEPSP}$ slope (Y axis) and FV amplitude (X axis). Symbols represent means \pm SEM from 5 to 6 mice per group. (ANOVA followed by Tukey's multiple comparisons test; "represents significant difference

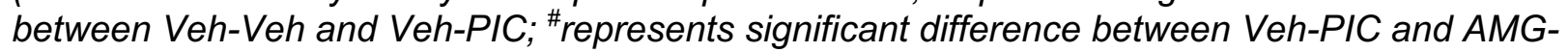
$P I C ;{ }^{* / \#} p \leq 0.05,{ }^{* * / \#} p \leq 0.01,{ }^{* * * \# \#} p \leq 0.001 ;$ ns = not significantly different). 
To determine if these alterations in basal synaptic transmission could result in altered synaptic plasticity, LTP and LTD were assessed. PIC challenge significantly enhanced LTP (Fig. 5A,B). When expressed as average fEPSP slope during 50-60 minutes following LTP induction, the enhancement amounted to $32 \%$ over the control value. AMG487 pretreatment negated this enhancement. PIC-challenged mice failed to exhibit LTD (Fig. 5C,D), whereas CXCR3 inhibition with AMG487 restored LTD to levels similar to vehicle-treated mice.

A

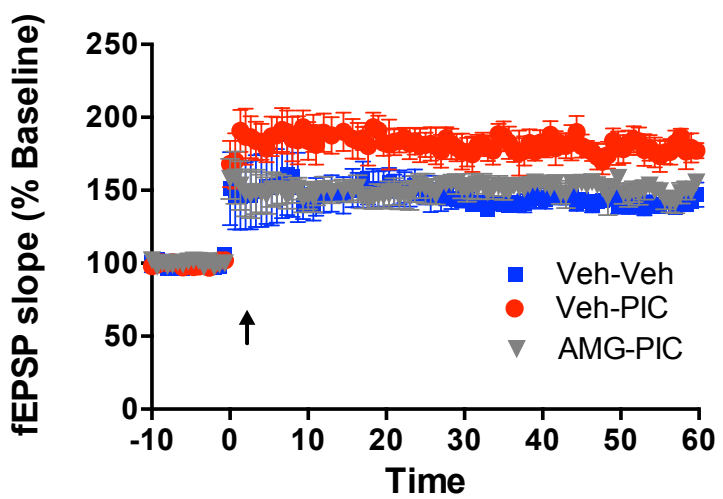

C

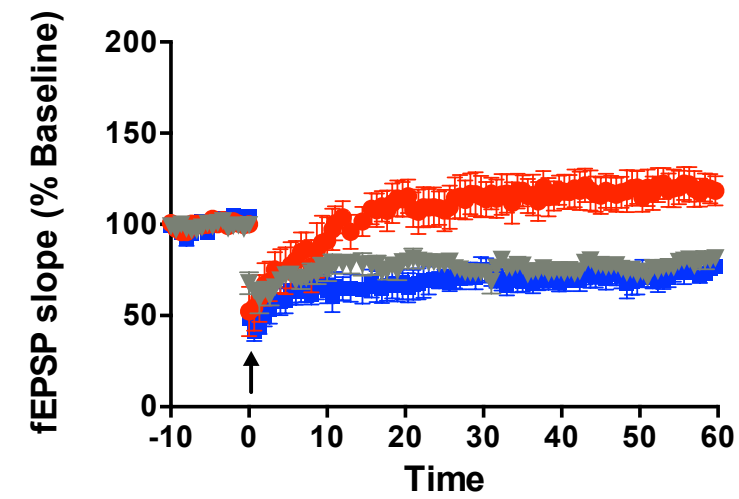

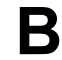

Long Term Potentiation

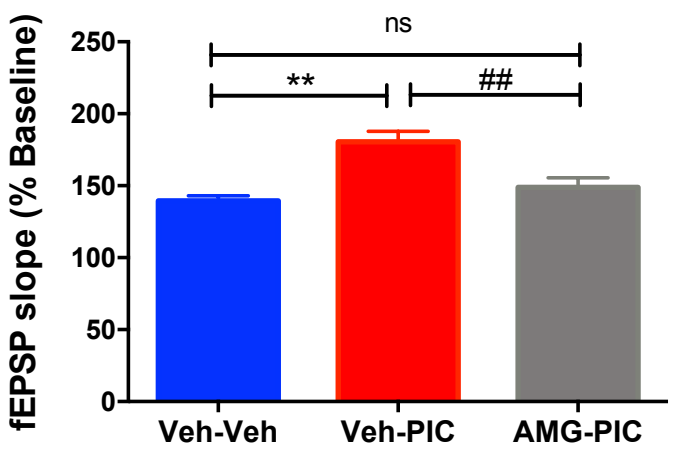

D

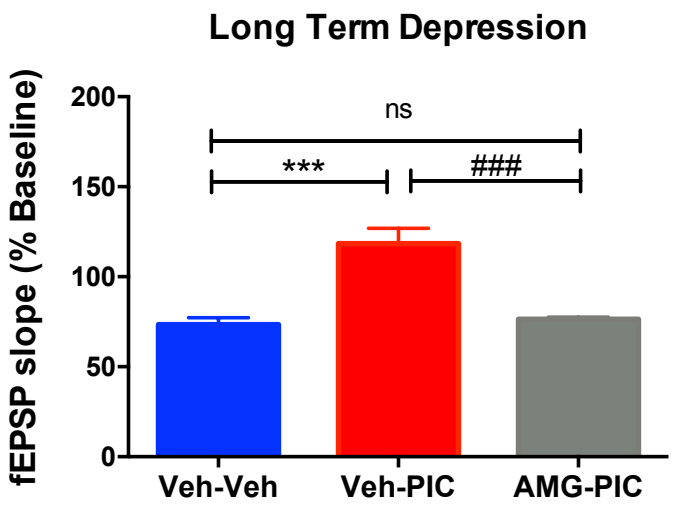

Figure 5. Synaptic plasticity in hippocampal slices. (A) Long term potentiation (LTP) represented by percent change in fEPSP slope over time. (B) LTP as represented by fEPSP slope during 50-60 minutes following LTP induction. (C) Long term depression (LTD) represented by percent change in fEPSP slope over time. (D) LTD as represented by fEPSP slope during 50-60 minutes following LTD induction. Symbols represent means \pm SEM from 5 to 6 mice per group. (ANOVA followed by Tukey's multiple comparisons test; " significant difference between Veh-Veh and Veh-PIC; ${ }^{*}$ represents significant difference between Veh-PIC and AMG-PIC; ${ }^{* \# p} p \leq 0.05,{ }^{* * \#} p \leq 0.01,{ }^{* * * \# \#} p \leq 0.001 ; n s=$ not significantly different). 
As previously demonstrated (Kirschman et al., 2011; Michalovicz and Konat, 2014; Hunsberger et al., 2017), PIC-challenge increased the susceptibility of mice to KA-induced seizures, as indicated by a $47 \%$ increase in cumulative seizure score (Fig. 6). CXCR3 blockade with AMG487 attenuated this PIC-mediated increase.

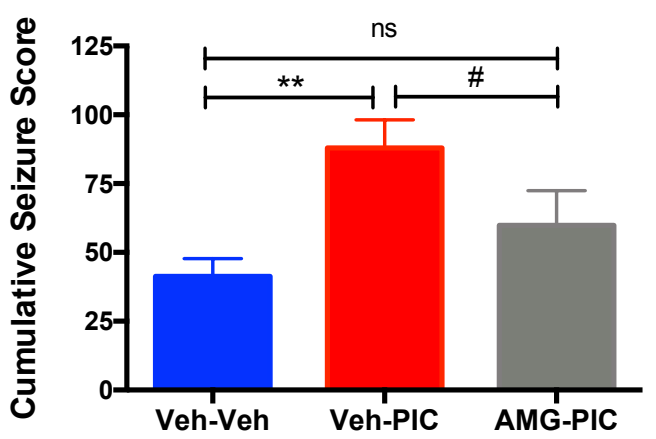

Figure 6. CXCR3 inhibition attenuates seizure
hypersensitivity induced by PIC challenge. Mice
received a bolus of i.c.v. injection of AMG 487 (3
mg/kg) or vehicle (VEH). Two hours later, the acute
antiviral response was induced by i.p. injection of 12
mg/kg of PIC in saline. Mice injected with $100 \mu \mathrm{L}$ of
saline served as vehicle controls. Twenty-four hours
after PIC injection, status epilepticus (SE) was
induced by subcutaneous injection of $12 \mathrm{mg} / \mathrm{kg}$ of
KA. Mice injected with saline in lieu of PIC served as controls (VEH). Seizures were expressed as cumulative seizure score. Symbols represent means \pm SEM from 3 to 6 mice per group. (ANOVA followed by Tukey's multiple comparisons test $;{ }^{*} p \leq 0.05,{ }^{* *} p \leq 0.01 ; n s=$ not significantly different).

\subsection{DISCUSSION}

We have previously shown that PIC challenge robustly upregulates cerebral expression of the Cxcl10 gene at the message (Fil et al., 2011; Michalovicz and Konat, 2014) and protein (Petrisko and Konat, 2017) levels. Here, we found neurons to be the predominant cell type responsible for CXCL10 generation in the brain. Interestingly, neuronal CXCL10 production has been shown to be induced by viral encephalitides (Rappert et al., 2004; Klein et al., 2005; Chai et al., 2015), indicating a commonality of neuronal response to systemic and central viral challenge. In concordance with previous study (Xia et al., 2000), only a subpopulation of astrocytes expressed CXCL10, although the contribution of these cells to the global production of the chemokine seems to be negligible. Resting microglia do not express CXCL10, but the expression is induced in microglia activated by viral infection of the brain (Chai et al., 2015). PIC challenge did not elicit microglial expression of CXCL10, indicating no development of proinflammatory phenotype of microglia. Our previous studies showing no effect of PIC challenge on the expression of cerebral iNOS (Konat et al., 2009) and CX3CL1 (Fil et al., 2011), the indices of neuroinflammation, buttress this contention.

CXCR3 is expressed constitutively on neurons and neuronal processes in the brain (Xia et al., 2000). Here, we found a similar pattern of expression of the receptor. Neuronal expression of both the receptor and its ligand indicates that the CXCL10/CXCR3 axis operates through an 
autocrine/paracrine neuronal signaling. Moreover, we found CXCR3 to be restricted to neurons, as both astrocytes and microglia were CXCR3-negative. Previously, CXCR3 expression was found in reactive, but nor quiescent astrocytes and microglia in a variety of CNS pathologies (Goldberg et al., 2001; Tanuma et al., 2006). Thus, the lack of glial CXCR3 expression provides further support for the notion that PIC challenge does not promote proinflammatory transformation of astrocytes and microglia.

In concordance with our previous electrophysiological study (Hunsberger et al., 2016), PIC challenge increased basal synaptic transmission, resulting in hyperexcitability of hippocampal circuits. The increased basal synaptic transmission likely occurred due to both increased presynaptic release and enhanced postsynaptic activity. The increase in presynaptic release in PIC-challenged mice possibly resulted from altered release mechanisms at the synapse because the data suggests no changes in presynaptic axon recruitment. Future studies will assess whether the enhanced postsynaptic activity is due to increased recruitment of glutamate receptors at the synapse or increased ion channel activity, or possibly both. Of note, we showed that alterations in both basal synaptic transmission and synaptic plasticity, as well as PICinduced seizure hypersensitivity, are abolished by the inhibition of CXCR3. These findings strongly indicate that the CXCL10/CXCR3 axis governs the induction of neuronal hyperexcitability. These results dovetail with previous in vitro studies that found CXCL10 to increase electrical activity of neurons in culture (Nelson and Gruol, 2004; Cho et al., 2009). Several possible mechanisms can be envisaged. For example, increased neuronal activity might result from the suppression of inhibitory GABAergic transmission, as a consequence of the downregulation of GAD65/67 and two GABAergic receptors, GABA $A_{B} R 1$ and $G A B A_{A} R \alpha 2$ (Cho et al., 2009). Interestingly, we found PIC challenge to downregulate the GABA receptor subunit $\varepsilon$ (GABRE) (Michalovicz and Konat, 2014). Moreover, the ligation of neuronal CXCR3 activates the ERK1/2 pathway (Xia et al., 2000), which phosphorylates GPH leading to the disruption of GABAergic synapses and their decreased transmission (Tyagarajan et al., 2013). Increased neuronal activity might also be attributed to the enhancement of excitatory glutamatergic transmission, as CXCL10 upregulates the expression of two glutamatergic receptors, NMDAR1 and mGluR2/3 (Cho et al., 2009). It is likely that both the attenuation of GABAergic and the enhancement of excitatory transmission contribute to shifting the neuronal balance toward excitation. Furthermore, additional, as yet unexplored, mechanisms might also mediate the development of hyperexcitability triggered by the neuronal CXCL10/CXCR3 axis signaling. 
In conclusion, we have demonstrated that anti-viral APR activates neuronal CXCL10/CXCR3 axis in the brain, and that this activation elicits hyperexcitability of neuronal circuits. Our results strongly suggest that the CXCL10/CXCR3 axis may plays a critical role in the comorbid effect of peripheral viral infections on the progression of major neuropathological diseases.

\section{Conflict of interest statement}

The authors declare that the research was conducted in the absence of any commercial or financial relationships that could be construed as a potential conflict of interest

\section{Author contribution statement}

MR and GK conceived and designed the experiments and wrote the manuscript. VS aided in experimental design. TP performed immunohistochemical and imaging experiments, analyzed the data, and edited the manuscript. JB, SS, SS, PP, YD, HH and RH performed the AMG inhibition experiments, analyzed the data, or edited the manuscript.

\section{Funding}

This work was supported by the WVU School of Medicine, Department of Biochemistry. The WVU Microscopy Imaging Facility is supported by NIH grants P20RR016440, P30GM103488, P20GM103434, and U54GM104942. 


\subsection{REFERENCES}

Andersen O, Lygner PE, Bergstrom T, Andersson M, Vahlne A (1993) Viral infections trigger multiple sclerosis relapses: a prospective seroepidemiological study. J Neurol 240:417422.

Bhattacharya D, Dunaway EP, Bhattacharya S, Bloemer J, Buabeid M, Escobar M, Suppiramaniam V, Dhanasekaran M (2015) Impaired ILK Function Is Associated with Deficits in Hippocampal Based Memory and Synaptic Plasticity in a FASD Rat Model. PLoS One 10:e0135700.

Bhattacharya S, Kimble W, Buabeid M, Bhattacharya D, Bloemer J, Alhowail A, Reed M, Dhanasekaran M, Escobar M, Suppiramaniam V (2017) Altered AMPA receptor expression plays an important role in inducing bidirectional synaptic plasticity during contextual fear memory reconsolidation. Neurobiol Learn Mem 139:98-108.

Buljevac D, Flach HZ, Hop WC, Hijdra D, Laman JD, Savelkoul HF, van Der Meche FG, van Doorn PA, Hintzen RQ (2002) Prospective study on the relationship between infections and multiple sclerosis exacerbations. Brain 125:952-960.

Caramia MD, Palmieri MG, Desiato MT, Boffa L, Galizia P, Rossini PM, Centonze D, Bernardi G (2004) Brain excitability changes in the relapsing and remitting phases of multiple sclerosis: a study with transcranial magnetic stimulation. Clin Neurophysiol 115:956-965.

Chai Q, She R, Huang Y, Fu ZF (2015) Expression of neuronal CXCL10 induced by rabies virus infection initiates infiltration of inflammatory cells, production of chemokines and cytokines, and enhancement of blood-brain barrier permeability. J Virol 89:870-876.

Cho J, Nelson TE, Bajova H, Gruol DL (2009) Chronic CXCL10 alters neuronal properties in rat hippocampal culture. J Neuroimmunol 207:92-100.

Cunningham C, Campion S, Teeling J, Felton L, Perry VH (2007) The sickness behaviour and CNS inflammatory mediator profile induced by systemic challenge of mice with synthetic double-stranded RNA (poly I:C). Brain Behav Immun 21:490-502.

Edwards S, Zvartau M, Clarke H, Irving W, Blumhardt LD (1998) Clinical relapses and disease activity on magnetic resonance imaging associated with viral upper respiratory tract infections in multiple sclerosis. J Neurol Neurosurg Psychiatry 64:736-741.

Eikermann-Haerter K (2014) Spreading Depolarization May Link Migraine and Stroke. Headache.

Esclapez M, Hirsch JC, Ben-Ari Y, Bernard C (1999) Newly formed excitatory pathways provide a substrate for hyperexcitability in experimental temporal lobe epilepsy. J Comp Neurol 408:449-460.

Fil D, Borysiewicz E, Konat GW (2011) A broad upregulation of cerebral chemokine genes by peripherally-generated inflammatory mediators. Metab Brain Dis 26:49-59.

George J, Bleasdale S, Singleton SJ (1997) Causes and prognosis of delirium in elderly patients admitted to a district general hospital. Age Ageing 26:423-427.

Goldberg SH, van der Meer P, Hesselgesser J, Jaffer S, Kolson DL, Albright AV, GonzalezScarano F, Lavi E (2001) CXCR3 expression in human central nervous system diseases. Neuropathology and applied neurobiology 27:127-138.

Holmes C (2013) Review: systemic inflammation and Alzheimer's disease. Neuropathol Appl Neurobiol 39:51-68.

Holmes C, El-Okl M, Williams AL, Cunningham C, Wilcockson D, Perry VH (2003) Systemic infection, interleukin 1beta, and cognitive decline in Alzheimer's disease. J Neurol Neurosurg Psychiatry 74:788-789.

Hunsberger HC, Konat GW, Reed MN (2017) Peripheral viral challenge elevates extracellular glutamate in the hippocampus leading to seizure hypersusceptibility. J Neurochem 141:341-346. 
Hunsberger HC, Wang D, Petrisko TJ, Alhowail A, Setti SE, Suppiramaniam V, Konat GW, Reed MN (2016) Peripherally restricted viral challenge elevates extracellular glutamate and enhances synaptic transmission in the hippocampus. J Neurochem 138:307-316.

Huynh W, Krishnan AV, Vucic S, Lin CS, Kiernan MC (2013) Motor cortex excitability in acute cerebellar infarct. Cerebellum 12:826-834.

Khedr EM, Ahmed MA, Darwish ES, Ali AM (2011) The relationship between motor cortex excitability and severity of Alzheimer's disease: a transcranial magnetic stimulation study. Neurophysiol Clin 41:107-113.

Kirschman LT, Borysiewicz E, Fil D, Konat GW (2011) Peripheral immune challenge with dsRNA enhances kainic acid-induced status epilepticus. Metab Brain Dis 26:91-93.

Klein RS, Lin E, Zhang B, Luster AD, Tollett J, Samuel MA, Engle M, Diamond MS (2005) Neuronal CXCL10 directs CD8+ T-cell recruitment and control of West Nile virus encephalitis. J Virol 79:11457-11466.

Konat GW, Borysiewicz E, Fil D, James I (2009) Peripheral challenge with double-stranded RNA elicits global up-regulation of cytokine gene expression in the brain. J Neurosci Res 87:1381-1388.

Lehmann TN, Gabriel S, Kovacs R, Eilers A, Kivi A, Schulze K, Lanksch WR, Meencke HJ, Heinemann U (2000) Alterations of neuronal connectivity in area CA1 of hippocampal slices from temporal lobe epilepsy patients and from pilocarpine-treated epileptic rats. Epilepsia 41 Suppl 6:S190-194.

Libbey JE, Fujinami RS (2010) Potential triggers of MS. Results Probl Cell Differ 51:21-42.

Michalovicz LT, Konat GW (2014) Peripherally restricted acute phase response to a viral mimic alters hippocampal gene expression. Metab Brain Dis 29:75-86.

Michalovicz LT, Lally BE, Konat GW (2015) Peripheral challenge with a viral mimic upregulates expression of the complement genes in the hippocampus. J Neuroimmunol 285:137142.

Michlmayr D, McKimmie CS (2014) Role of CXCL10 in central nervous system inflammation. Int J Interferon Cytokine Mediators Res 6:1-18.

Morrison RS, Wenzel HJ, Kinoshita Y, Robbins CA, Donehower LA, Schwartzkroin PA (1996) Loss of the p53 tumor suppressor gene protects neurons from kainate-induced cell death. J Neurosci 16:1337-1345.

Murray AM, Levkoff SE, Wetle TT, Beckett L, Cleary PD, Schor JD, Lipsitz LA, Rowe JW, Evans DA (1993) Acute delirium and functional decline in the hospitalized elderly patient. J Gerontol 48:M181-M186.

Nee LE, Lippa CF (1999) Alzheimer's disease in 22 twin pairs--13-year follow-up: hormonal, infectious and traumatic factors. Dement Geriatr Cogn Disord 10:148-151.

Nelson TE, Gruol DL (2004) The chemokine CXCL10 modulates excitatory activity and intracellular calcium signaling in cultured hippocampal neurons. J Neuroimmunol 156:7487.

Palasik W, Fiszer U, Lechowicz W, Czartoryska B, Krzesiewicz M, Lugowska A (2005) Assessment of relations between clinical outcome of ischemic stroke and activity of inflammatory processes in the acute phase based on examination of selected parameters. Eur Neurol 53:188-193.

Parameshwaran K, Buabeid MA, Bhattacharya S, Uthayathas S, Kariharan T, Dhanasekaran M, Suppiramaniam V (2013) Long term alterations in synaptic physiology, expression of beta2 nicotinic receptors and ERK1/2 signaling in the hippocampus of rats with prenatal nicotine exposure. Neurobiol Learn Mem 106:102-111.

Penzes P, Cahill ME, Jones KA, VanLeeuwen JE, Woolfrey KM (2011) Dendritic spine pathology in neuropsychiatric disorders. Nature neuroscience 14:285-293.

Petrisko T, Konat G (2017) Peripheral viral challenge triggers hippocampal production of inflammatory proteins. Metab Brain Dis 32:1249-1254. 
Rappert A, Bechmann I, Pivneva T, Mahlo J, Biber K, Nolte C, Kovac AD, Gerard C, Boddeke HW, Nitsch R, Kettenmann H (2004) CXCR3-dependent microglial recruitment is essential for dendrite loss after brain lesion. J Neurosci 24:8500-8509.

Rossi S, Furlan R, De C, V, Motta C, Studer V, Mori F, Musella A, Bergami A, Muzio L, Bernardi G, Battistini L, Martino G, Centonze D (2012) Interleukin-1beta causes synaptic hyperexcitability in multiple sclerosis. Ann Neurol 71:76-83.

Scharfman HE (2012) "Untangling" Alzheimer's disease and epilepsy. Epilepsy Curr 12:178183.

Scheid R, Teich N (2007) Neurologic manifestations of ulcerative colitis. Eur J Neurol 14:483493.

Tanuma N, Sakuma H, Sasaki A, Matsumoto Y (2006) Chemokine expression by astrocytes plays a role in microglia/macrophage activation and subsequent neurodegeneration in secondary progressive multiple sclerosis. Acta Neuropathol 112:195-204.

Tellez-Zenteno JF, Matijevic S, Wiebe S (2005) Somatic comorbidity of epilepsy in the general population in Canada. Epilepsia 46:1955-1962.

Tyagarajan SK, Ghosh H, Yevenes GE, Imanishi SY, Zeilhofer HU, Gerrits B, Fritschy JM (2013) Extracellular signal-regulated kinase and glycogen synthase kinase 3beta regulate gephyrin postsynaptic aggregation and GABAergic synaptic function in a calpain-dependent mechanism. J Biol Chem 288:9634-9647.

Verrotti A, Tocco AM, Coppola GG, Altobelli E, Chiarelli F (2009) Afebrile benign convulsions with mild gastroenteritis: a new entity? Acta Neurol Scand 120:73-79.

Vlkolinsky R, Siggins GR, Campbell IL, Krucker T (2004) Acute exposure to CXC chemokine ligand 10, but not its chronic astroglial production, alters synaptic plasticity in mouse hippocampal slices. J Neuroimmunol 150:37-47.

Xia MQ, Bacskai BJ, Knowles RB, Qin SX, Hyman BT (2000) Expression of the chemokine receptor CXCR3 on neurons and the elevated expression of its ligand IP-10 in reactive astrocytes: in vitro ERK1/2 activation and role in Alzheimer's disease. J Neuroimmunol 108:227-235.

Yener GG, Basar E (2013) Biomarkers in Alzheimer's disease with a special emphasis on event-related oscillatory responses. Suppl Clin Neurophysiol 62:237-273. 


\section{CHAPTER 4: UNPUBLISHED DATA}

\subsection{SPATIOTEMPORAL CXCL10 EXPRESSION INDUCED BY PIC CHALLENGE}

\section{Supplemental Data to Chapter 3}

Fig. 1 in Chapter 3 depicts a robustly increased generation of neuronal CXCL10 in hippocampal CA1 region and the motor cortex (CTX), $24 \mathrm{hr}$ after PIC challenge. This supplemental study was designed to determine time course and regional expression of neuronal CXCL10. As shown in Fig. 1, low levels of neuronal CXCL10 were observed in CA1, CA3, DG and CTX in saline injected mice. In the hippocampal subregions, CXCL10 staining increased with time reaching maximum at $12 \mathrm{~h}$, decreased slightly at $24 \mathrm{hr}$, and dropped to the basal level at $96 \mathrm{hr}$ after PIC challenge. This temporal expression of the chemokine is congruent with the generation of hippocampal CXCL10 determined by ELISA (Chapter 2). The cortex featured a similar kinetics, although the peak intensity of CXCL10 staining was maintained between 12 and $24 \mathrm{hr}$ post PIC challenge. Moreover, the upregulation of CXCL10 expression was not restricted to the four regions studied, but was evident throughout the entire brain (Fig. 2).

\section{CA1}

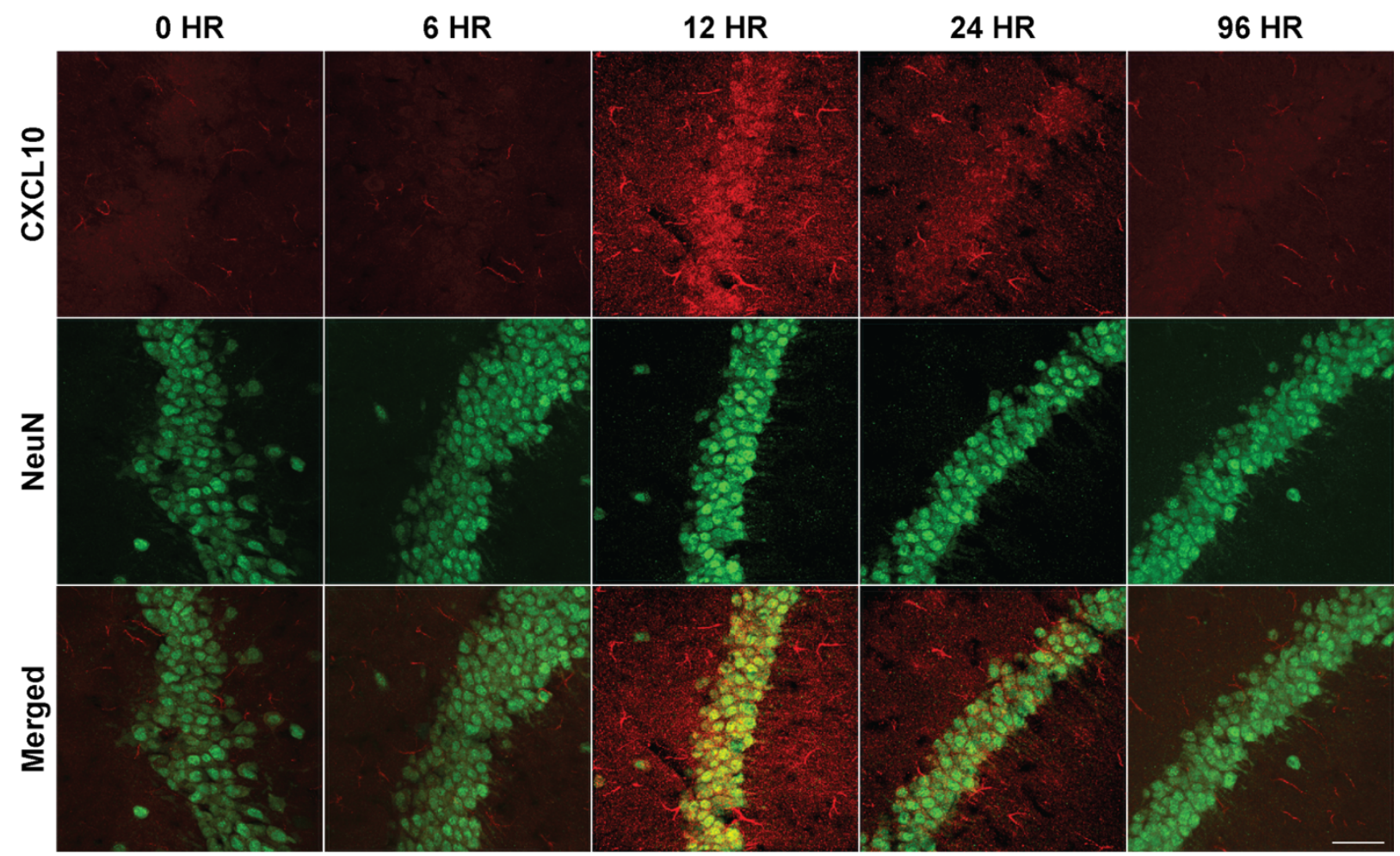




\section{CA3}
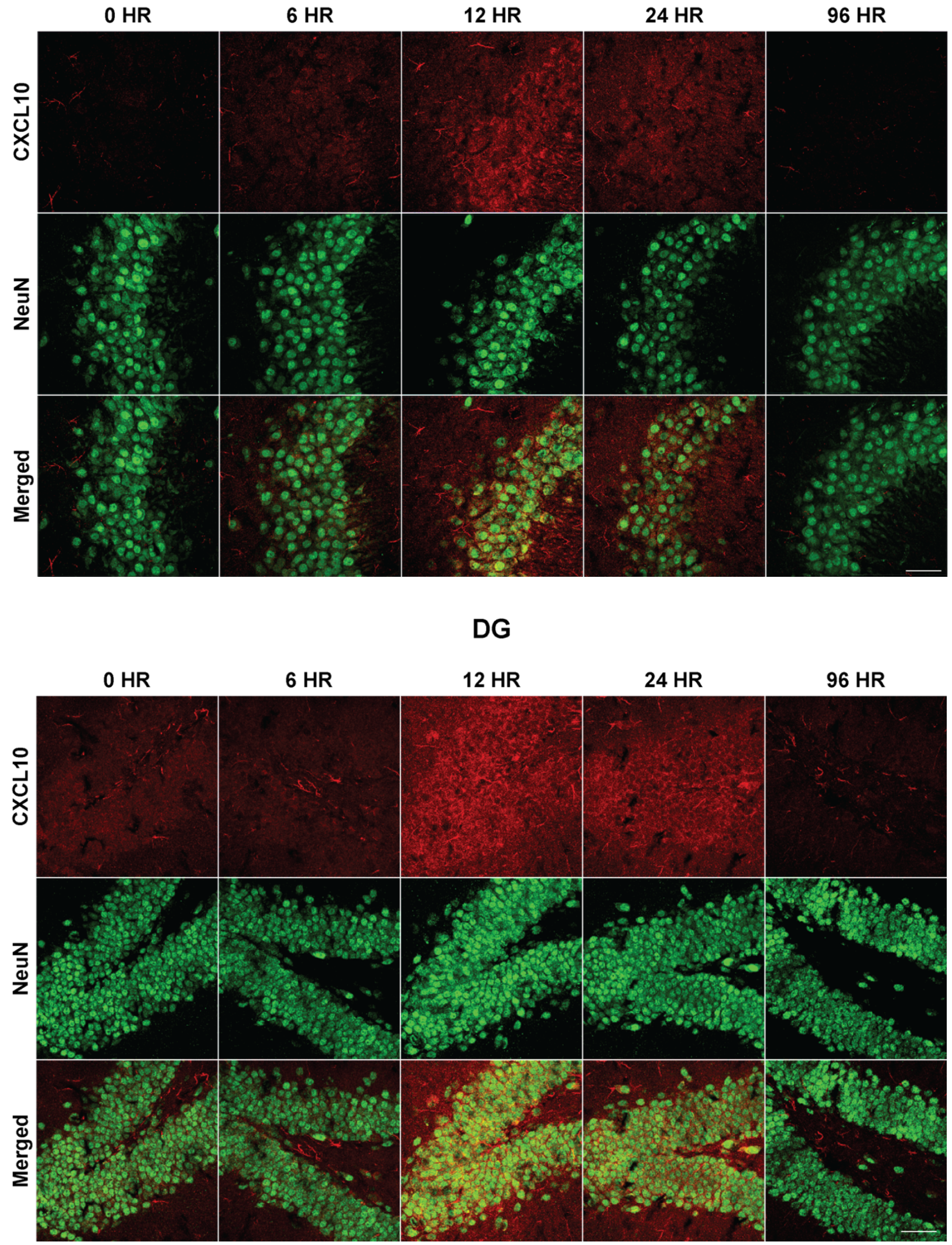


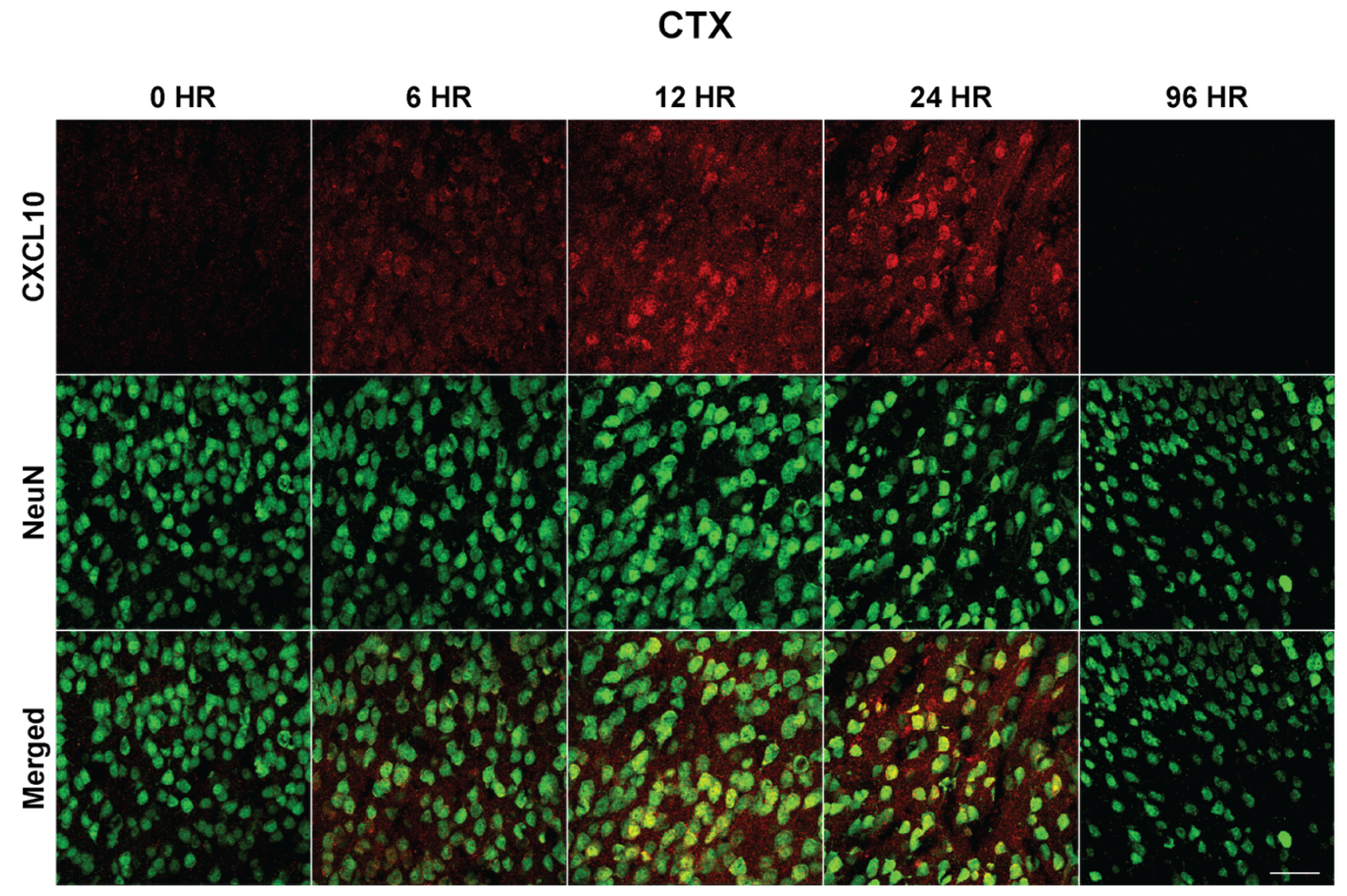

Figure 1. Time course of neuronal CXCL10 expression in different brain regions following PIC challenge. The animal treatment and microscopic analysis were performed as described in Chapter 3. CXCL10 is stained red, while neurons are stained green. CA1, cornu ammonis 1; CA3, cornu ammonis 3; DG, dentate gyrus; CTX, motor cortex. Confocal images were captured at 60X magnification. Scale bars represent $50 \mu \mathrm{m}$. 


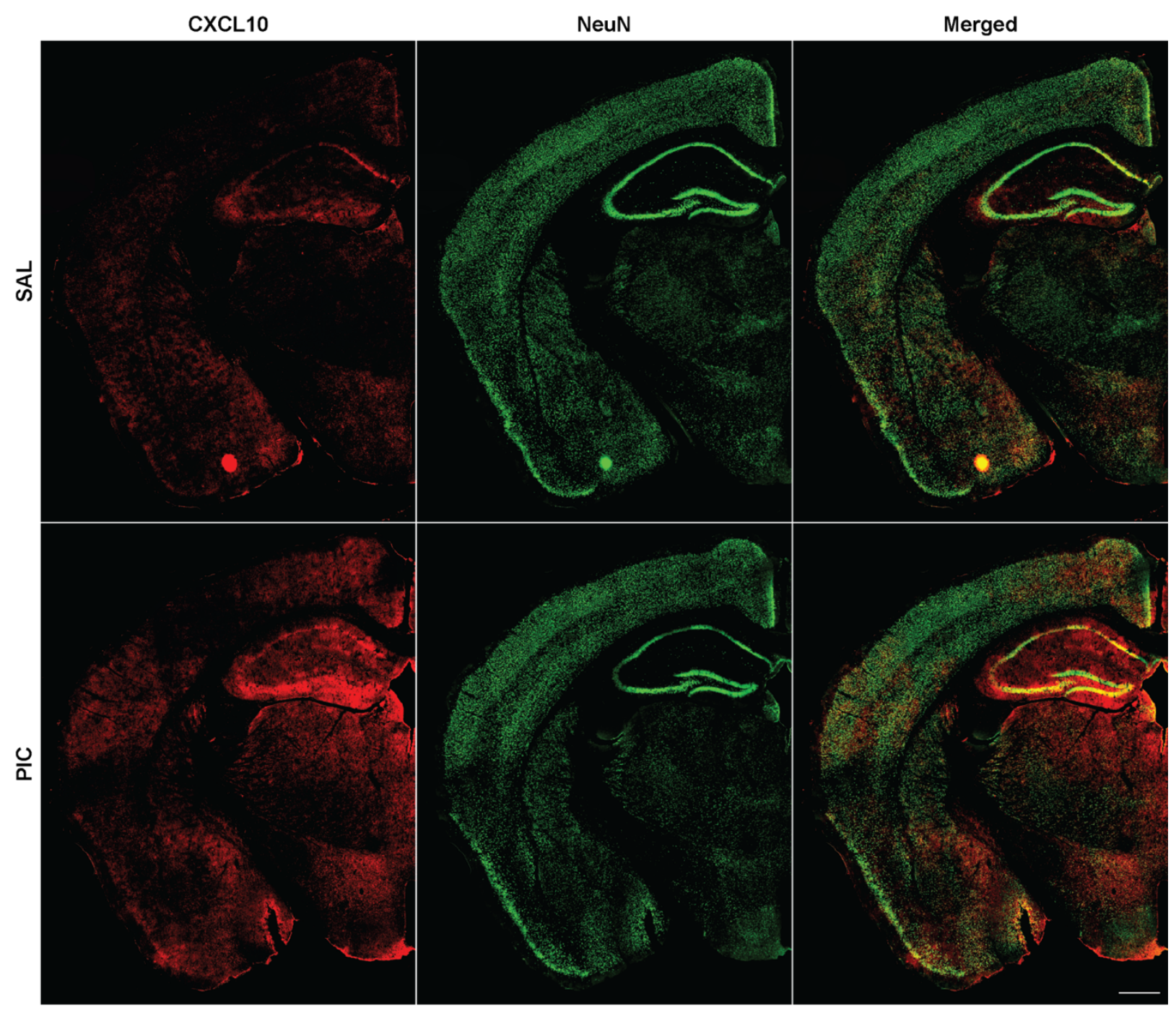

Figure 2. Cerebral generation of CXCL10 induced by PIC challenge. The animal treatment and immunohistochemistry were as described in Fig. 1. Epifluorescent images of coronal brain sections were captured at 10X magnification. Scale bars represent $500 \mu \mathrm{m}$.

These results indicate that peripheral PIC challenge induces transient generation of CXCL10 by neurons, and that this neuronal response is global, i.e., entails all brain regions. However, kinetics of this response may differ between brain regions as exemplified by the hippocampus vs. cortex regarding the chemokine generation at 12 vs. $24 \mathrm{hr}$ post PIC. 


\title{
4.2 NEURONAL C-FOS EXPRESSION AS AN INDEX OF HYPEREXCITABILITY
}

\author{
Manuscript in Preparation
}

\subsubsection{INTRODUCTION}

C-fos is an immediate-early gene (IEG) that undergoes a dramatic, yet transient, upregulation in neurons in response to a variety of depolarizing stimuli including neurotransmitters (Kawasaki et al., 2009; Shevelkin et al., 2012), neurotrophic factors (Baille-Le Crom et al., 1996; Joo et al., 2016), cytokines (Srinivasan et al., 2004), and direct stimulation (Sheng et al., 1990; Thompson et al., 1995; Fields et al., 1997). Neuronal c-fos expression profoundly increases in the hippocampus and cortex during seizures (Morgan et al., 1987; Baille-Le Crom et al., 1996; Herrera and Robertson, 1996; Zhang et al., 2002; Dudek, 2006; Barros et al., 2015). As such, the use of c-fos immunoreactivity has become increasingly common, because it allows for much faster and more cost-effective measure of neuronal activity than electrophysiology (Bullitt, 1990; Kovacs, 2008; Kawashima et al., 2014; Malhi et al., 2014)

This study was undertaken to determine whether c-fos immunoreactivity is a viable technique to examine PIC challenge-induced hyperexcitability.

\subsubsection{METHODS}

\subsubsection{Drug Administration}

Cerebral CXCR3 inhibition in mice was achieved as described in Chapter 3 with some modifications. In particular, we used unilateral i.c.v. injection AMG-487, and the coordinates from bregma were anteroposterior $0.45 \mathrm{~mm}$, mediolateral $+1.0 \mathrm{~mm}$, and dorsoventral $-3.6 \mathrm{~mm}$. Because AMG-487 solution was prepared in ACSF containing 10\% of DMSO, respective controls were injected with equivolume amounts of $10 \%$ DMSO in ASCF. After 2 hours, the animals were i.p. injected with PIC in saline, or saline alone (controls) as described in Chapters 2 and 3. Six hours after PIC challenge, the rearing test was performed as in Chapters 2 and 3. Additionally, mice were weighed at 0 and $24 \mathrm{hr}$ after PIC challenge.

\subsubsection{Microscopy}

Immunohistochemistry was performed $24 \mathrm{hr}$ post PIC challenge as previously described (Chapter 3) using mouse-anti-NeuN (1:500; Millipore) and rabbit-anti-c-fos (1:10,000 Abcam) 
primary antibodies. Confocal images were obtained using a 40X objective with a Nyquist value of $0.23 \mu \mathrm{m}$ at a resolution of $512 \times 512$ pixels. 3D projections were rendered using NIS Elements Advanced Research imaging software (Nikon Instruments, Melville, NY) unless otherwise indicated. The same LUT settings were applied to all images.

\subsubsection{Neuronal Expression of c-fos}

C-fos positive neurons were quantified using Nikon Elements General Analysis 3 (GA3). Briefly, de-identified confocal z-stacks were imported into Nikon Elements and GA3. Background subtraction and gaussian smoothing were applied prior to 3D thresholding for neurons and c-fos modeling. Additional background staining was eliminated by a setting minimum diameter requirement of $\sim 7 \mu \mathrm{m}$ for neurons and $\sim 3 \mu \mathrm{m}$ for c-fos in 3D. Parameters were adjusted on an image-by-image basis to maximize the accuracy of the modeling with the researcher blinded to the conditions. The total number of neurons, and the percentage of $c$-fos containing neurons within the image were then counted. The intensity of c-fos within individual neurons was calculated using the original, unmodified fluorescent data.

\subsubsection{Statistical Analysis}

The data was analyzed by one-way ANOVA followed by the Bonferroni post-hoc test. Differences between groups were considered significant at $p<0.05$.

\subsubsection{RESULTS AND DISCUSSION}

Because the high density of neurons within the hippocampus prevents accurate quantitation of neurons, we performed these experiments in the motor cortex. Fig 1. depicts c-fos-expression in neurons at different time points after PIC challenge. Only a few c-fos+ neurons were present at $0 \mathrm{hr}$. At $24 \mathrm{hr}$, the population of c-fos+ neurons and the intensity of c-fos staining dramatically increased. A slight increase was also evident at $6 \mathrm{hr}$ post PIC challenge. Quantitative analysis of c-fos+ neurons by General Analysis 3 and one-way ANOVA showed the effect of time was significant $[F(3,12)=20.582, p<0.001]$. Post-hoc analysis (Fig. $2 A)$ revealed that the percentages of c-fos positive neurons did not significantly differ between $0(4.48 \% \pm 3.19), 6$ $(17.53 \% \pm 3.68)$ and $12(11.84 \% \pm 4.08) \mathrm{hr}$ after PIC challenge. However, the percentage of c-fos+ neurons at $24 \mathrm{hr}(48.25 \% \pm 4.62)$ was significantly higher that the value at $0(p<0.001), 6$ $(p<0.004)$ and $12 \mathrm{hr}(p<0.001)$. The increase at $24 \mathrm{vs.} 0 \mathrm{~h}$ was approximately 10 -fold. This increase was concomitant with an augmented intensity of c-fos staining in individual neurons Fig. 2B. Total number of neurons per image did not differ between groups. 
As previously shown by electrophysiology in Chapter 3, neuronal hyperexcitability induced by PIC challenge is profoundly attenuated by the inhibition of CXCR3 receptor. Therefore, we tested whether neuronal c-fos expression is also CXCR3-dependent.

Initially, we determined whether the i.c.v. injections of the CXCR3 inhibitor, AMG-487, alters PIC-induced sickness behavior by measuring the loss of body weight, and by the rearing test. No difference in body weight loss was observed between DMSO-SAL and AMG-SAL injected mice (Fig. 1). Consistent with previous study (Cunningham et al., 2007), PIC challenge resulted in an approximately $10 \%$ weight body loss during 24 hours. No significant difference was observed between DMSO-PIC and AMG-PIC treated animals.

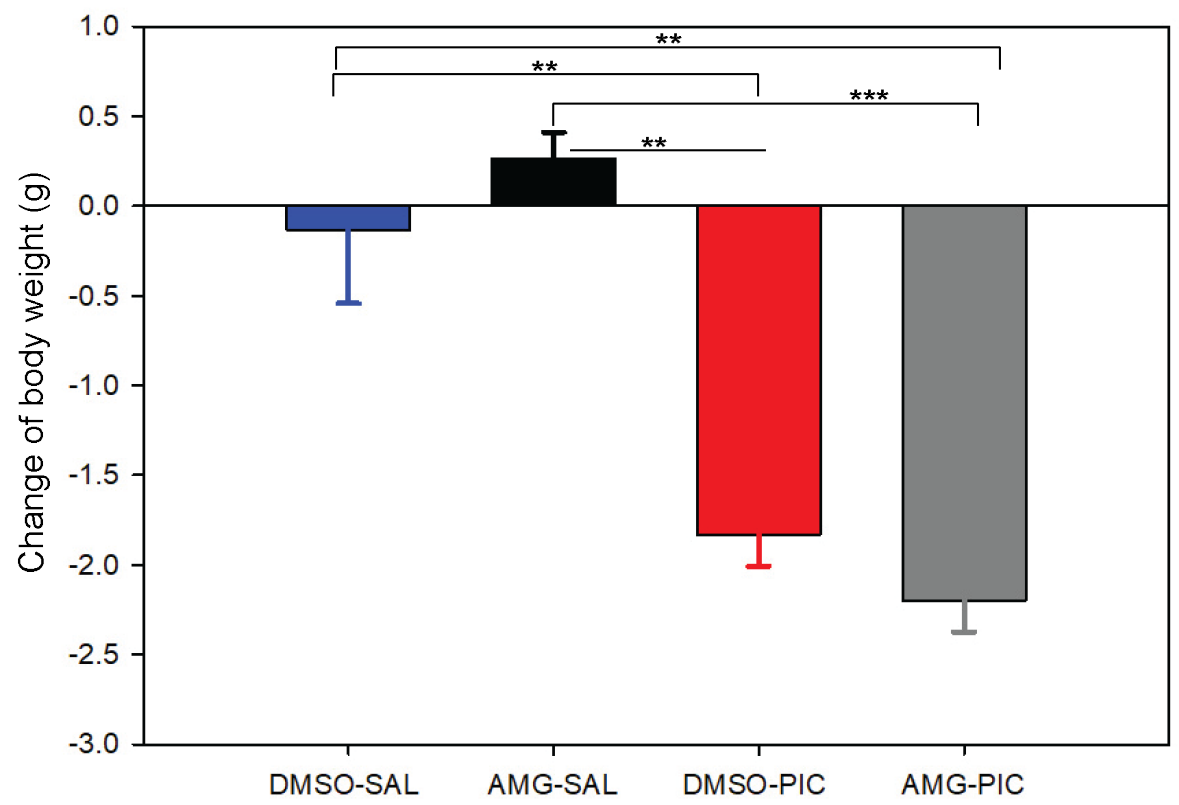

Figure 1. Effect of CXCR3 Inhibition on body weight. Mice received i.c.v. injection of AMG487 or DMSO. Two hours later, mice were i.p. injected with PIC or saline (SAL) as described in Methods. Mice were weighed at 0 and $24 \mathrm{hr}$ after PIC challenge to calculate body weight loss. Bars represent means \pm SEM from 3 mice per group. ${ }^{*} p<0.05,{ }^{* *} p<0.01,{ }^{* * *} p<0.001$.

Our highly sensitive rearing test (Michalovicz and Konat, 2014) quantifies the loss of locomotor activity, motivation, and exploratory behavior by measuring the number of times animals stand on their hind limbs and reach a height of at least $8 \mathrm{~cm}$ during a 15-minute period. This test was performed six hr after PIC injection, as this time point corresponds to the peak of PIC challengeinduced sickness behavior. Significant differences between groups was observed $[F(3,11)=$ 
13.346, $p=0.002]$. DMSO+PIC (16.0 \pm 5.69$)$ animals reared significantly less than DMSO+SAL $(53.33 \pm 10.71 ; p=0.008)$ animals (Fig. 2). There was no difference in the number of rears between DMSO+PIC and AMG+PIC (5.00 \pm 1.53$)$ animals nor between AMG+SAL and AMG+PIC treatments. Surprisingly, AMG-SAL (26.33 \pm 3.93$)$ treated animals reared significantly less compared to DMSO-SAL animals $(p=0.008)$.

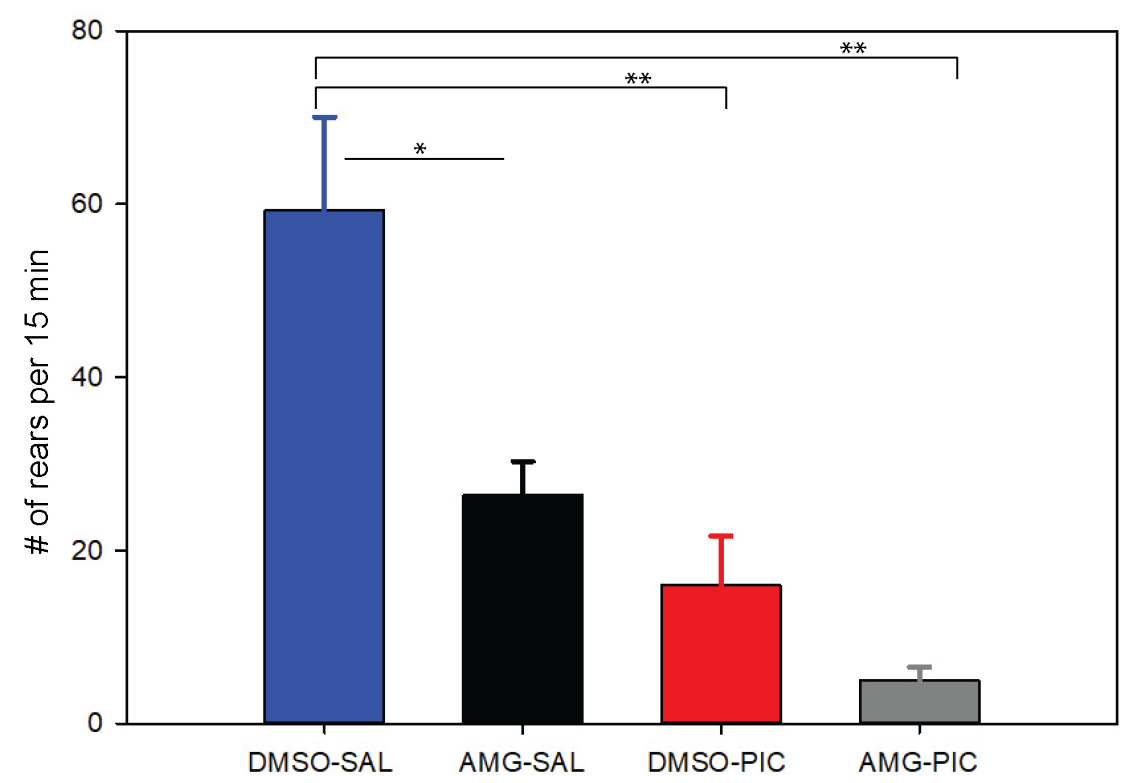

Figure 2. Effect of CXCR3 Inhibition on rearing behavior. Mice received i.c.v. injection of $A M G-487$ or DMSO. Two hours later, mice were i.p. injected with PIC or saline (SAL) as described in Methods. Six hours after PIC challenge, the animals were examined to the rearing test. The number of rears in a 15 minute period were recorded. Bars represent means \pm SEM from 3 mice per group. ${ }^{*} p<0.05$, ${ }^{* *} p<0.01$

Together, these results show that i.c.v. injected AMG-487 does not impair somatic functions as seen from the lack of effect of the body weight. However, the inhibitor has a profound effect on the CNS function as seen from the reduction of rearing events. Interestingly, CXCR3-deficient mice were previously reported not to develop depressive behavior following PIC challenge (Blank et al., 2016). The discrepancy between this and our result might be due to the different models used, i.e., transgenic vs. pharmacological reduction of CXCR3 activity. Additionally, we are unable to determine the exact amount of AMG-487 relative to the extracellular CXCL10 concentration, as our previous study profiling chemokine protein levels (Chapter 2) examined whole hippocampi consisting of both extracellular and intracellular CXCL10. As immunohistochemistry studies have shown the majority of CXCL10 expression is intracellular (Chapters, 3 and 4.1), we are unable to know exactly how much CXCL10 is present in the extracellular fluid throughout the brain. However, it must be noted that while the dose of AMG- 
487 utilized throughout this study may result in off target effects on additional receptors that may account for the observed results.

Representative images presented in Fig. 3 demonstrate an overt reduction of the c-fos+ neuronal population brought about by AMG-487. Quantitative analysis revealed a significant effect of group on the percentage of $c$-fos positive neurons $[F(3,14)=43.700$, p<0.001] (Fig. 4A). DMSO-PIC $(28.454 \% \pm 1.61)$ animals had significantly more c-fos positive neurons compared to DMSO-SAL (14.019 $\pm 1.38 ; p<0.001)$, AMG-SAL $(8.474 \% \pm 2.11 ; p<0.001)$ and AMG-PIC (4.373\% \pm 0.26 ; $p<0.001)$. There was no difference between DMSO-SAL and AMGSAL, indicating that AMG pretreatment by itself did not significantly alter c-fos expression. Additionally, AMG pretreatment returned AMG-PIC c-fos expression back to AMG-SAL levels $(p=0.423)$. Total number of neurons per image did not differ between groups. The point plot displaying the intensity of c-fos staining in individual neurons is shown in Fig. 4B.

\section{CTX}

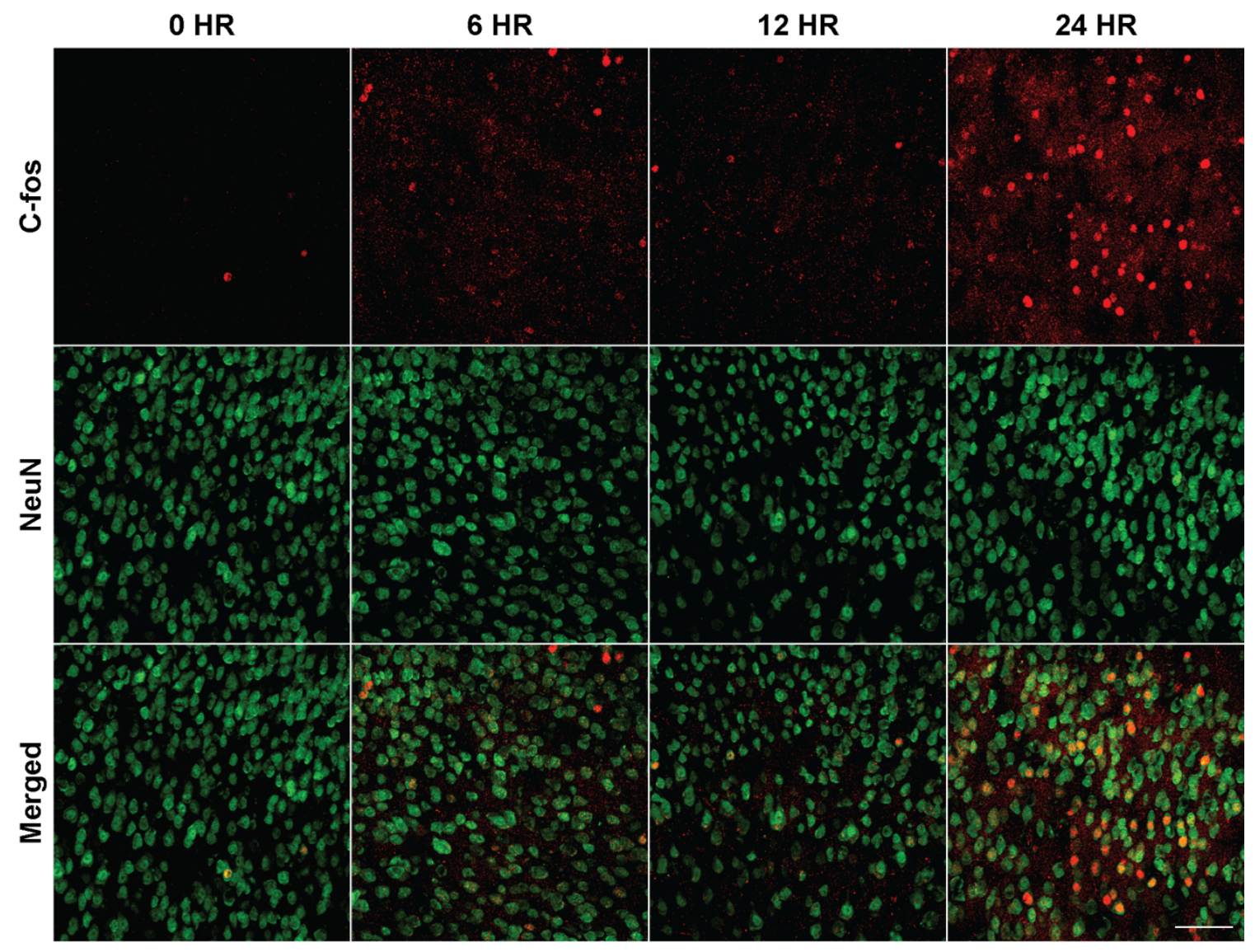


Figure 3. Time course of neuronal c-fos expression following PIC challenge. At various time points after PIC challenge, brains were analyzed by confocal microscopy (see Methods). Images were captured with a 40X objective. Scale bars represent $40 \mu \mathrm{m}$.
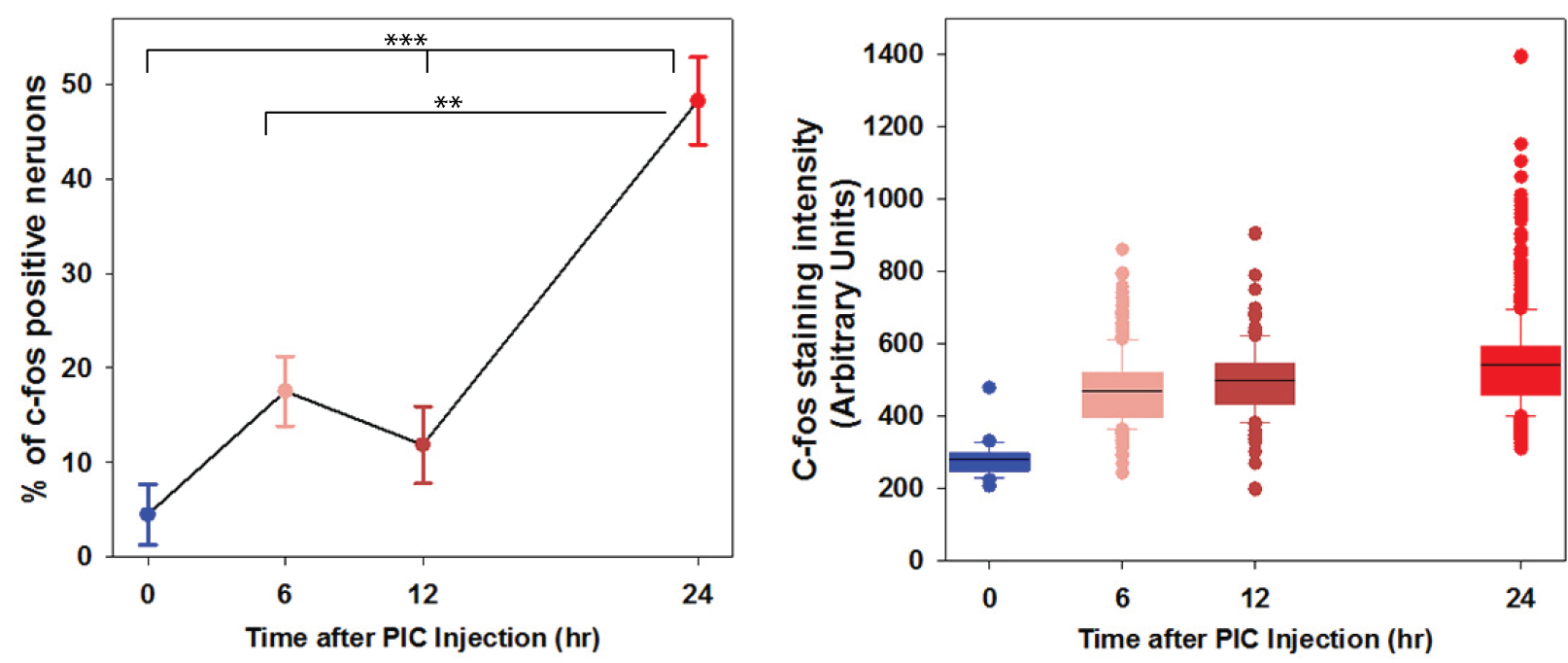

Figure 4. Temporal expression of neuronal c-fos instigated by PIC challenge. $3 D$ modeling of neurons and c-fos was performed using General Analysis 3 in Nikon Elements. A) The percentage of neurons expressing c-fos at each time point. Bars represent means \pm SEM from 3 mice per group. ${ }^{* * *} p<0.001$ compared to $0 \mathrm{HR}$. ${ }^{\# \#} p<0.01$ between pairwise comparison between non-zero HR time points $B$ ) Box and whisker displaying the range of mean c-fos intensities within individual c-fos positive neurons.

While c-fos is typically expressed within minutes of increased neuronal activity, and in seizure models sustained for approximately 12 hours (Baille-Le Crom et al., 1996; Barros et al., 2015), here, we found that the increase in the number of c-fos+ neurons is profoundly delayed by $24 \mathrm{hr}$ after PIC challenge. This apparent discrepancy can be explained by the fact that the aforementioned studies induced neuronal hyperactivity, while we induce neuronal hyperexcitability. PIC challenge does not result in seizures but renders the neuronal circuits more prone to the induction of seizures. Thus, the neuronal activity is not, but the propensity of neurons to fire is increased. The delayed increase in c-fos+ neurons indicates a complex transcriptional, translational and posttranslational process that ultimately lead to hyperexcitability. These processes should be addressed in future studies.

The increased expression of neuronal c-fos is concordant with our electrophysiological experiments (Chapter 3) showing neuronal hyperexcitability (Michalovicz, 2013; Hunsberger et al., 2016; Hunsberger et al., 2017). Moreover, the attenuation of neuronal c-fos expression by CXCR3 inhibition further confirms our electrophysiological results. Overall, these results indicate 
that cortical c-fos immunoreactivity is a viable technique to examine neuronal hyperexcitability induced by PIC challenge, and possibly in other experimental and clinical conditions.

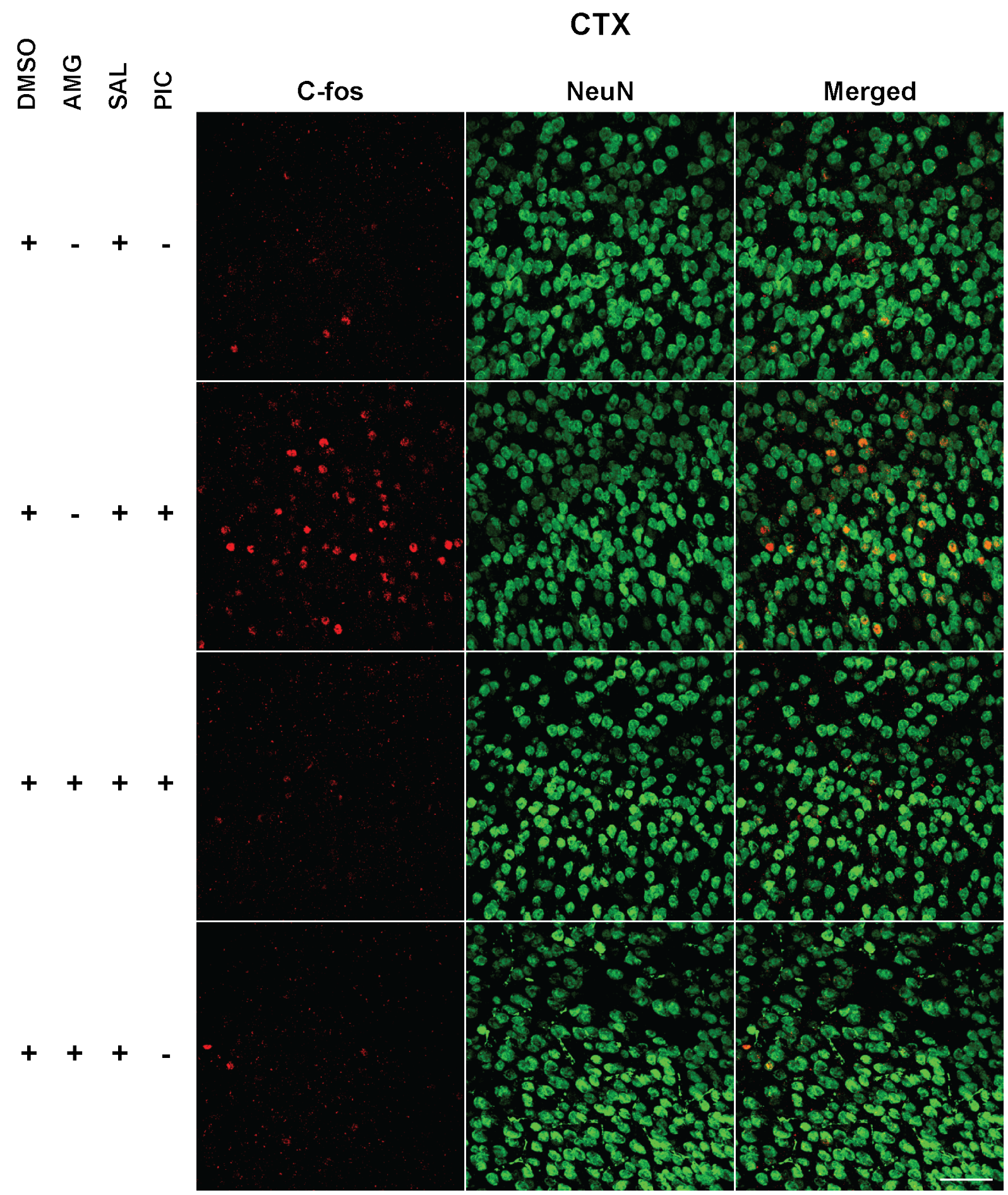

Figure 5. Effect of CXCR3 inhibition on PIC challenge - induced neuronal c-fos expression. Mice received a i.c.v. injection of CXCR3 inhibitor, AMG-487 (AMG), or the vehicle (DMSO). Two hours later, mice were i.p. injected with PIC (PIC), or the vehicle (SAL). After 24 
$\mathrm{hr}$, the cortex was analyzed by immunohistochemistry using antibodies for c-fos (red) and neurons (green). Confocal images of the cortex were captured with a 40x objective. Scale bars represent $40 \mu \mathrm{m}$.

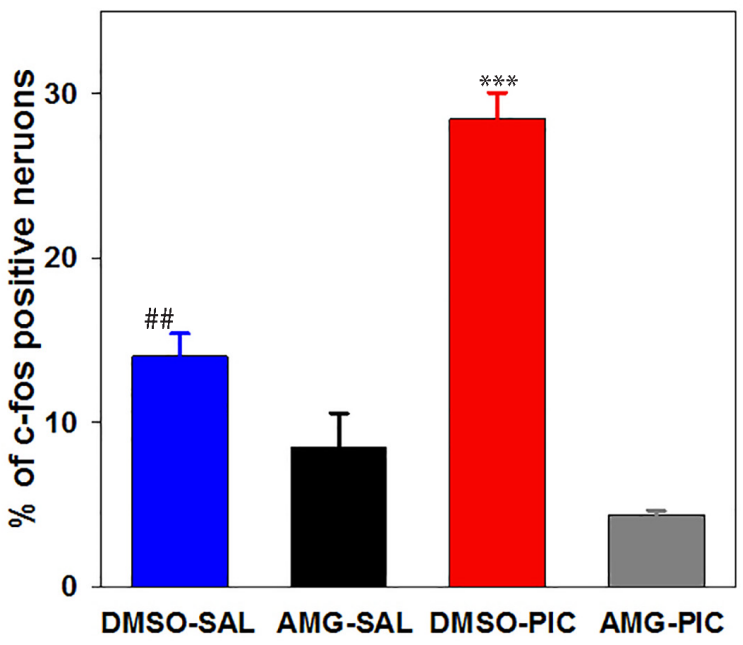

Condition

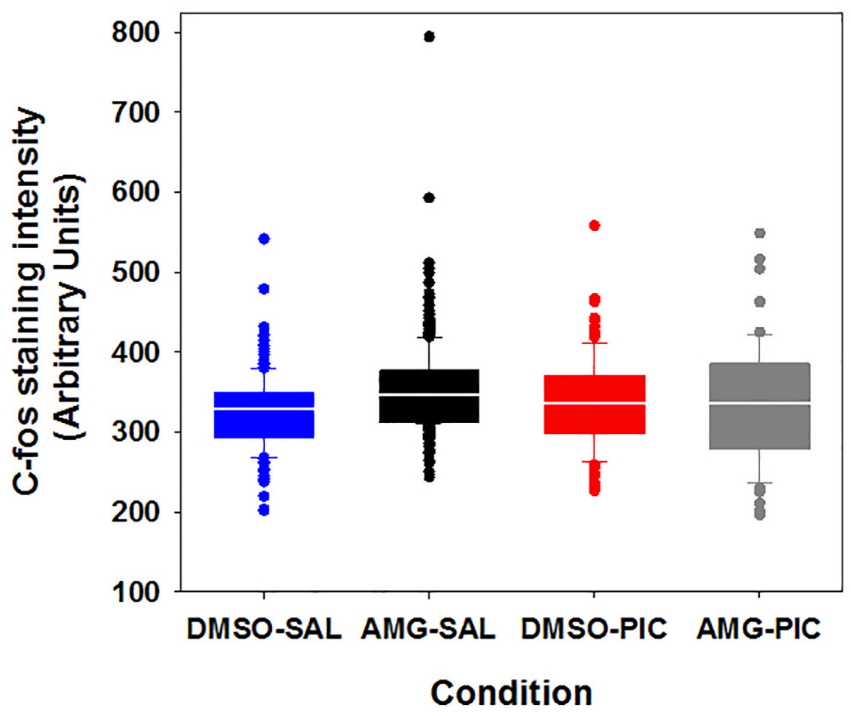

Condition

Figure 6. Effect of CXCR3 inhibition on c-fos expression. 3D modeling of neurons and c-fos was performed using General Analysis 3 in Nikon Elements to determine A) the percentage of neurons expressing c-fos following i.c.v injections of AMG-487 or DMSO 2 hours prior to i.p. injections of saline or PIC.A) The percentage of neurons expressing c-fos at each time point. ${ }^{* * *} p<0.001$ compared to DMSO-PIC. ${ }^{\#} p<0.01$ B) Box and whisker displaying the range of mean $c$-fos intensities within individual c-fos positive neurons. 


\title{
4.3. RESPONSE OF MICROGLIA TO PIC CHALLENGE
}

\author{
Manuscript in Preparation
}

\subsubsection{INTRODUCTION}

Microglia are integral components of the central nervous system. Their primary function entails the surveillance of tissue environment (Nimmerjahn et al., 2005), and the initiation of immune responses following insult or injury (Aldskogius et al., 1999; Cunningham, 2013; Franco and Fernandez-Suarez, 2015; Morrison et al., 2017). They are also critical for proper neurodevelopment (Cunningham et al., 2013; Schafer et al., 2013), and the maintenance of synapses (Beattie et al., 2002; Hong et al., 2016a; Akiyoshi et al., 2018) either via physical contact (Chen et al., 2014; Akiyoshi et al., 2018), or by secreting modulatory factors (Beattie et al., 2002; Ji et al., 2013). When activated, microglia can take on a variety of morphological shapes and functions, ranging from pro-inflammatory states, which promote inflammation, to anti-inflammatory states, which assist in the repair of damaged tissue. Due to their importance as the main resident immune cell of the brain, as well as their ability to modulate neuronal activity, understanding how microglia react to peripheral PIC-challenge can provide insight into mechanisms underlying the induction of hyperexcitability.

The goal of this study was to determine the response of microglia to peripheral PIC injection. We examined the activation and proliferation of microglia, as well as their interaction with neurons. We also examined the role of CXCR3 in microglial responses to PIC challenge.

\subsubsection{METHODS}

\subsubsection{Drug Administration}

Peripheral PIC challenge was induced in C57BL/6 J mice as previously described (Chapters 2, 3). CXCR3 inhibition was achieved by intracerebroventricular (i.c.v.) injection of $3.0 \mathrm{mg} / \mathrm{kg}$ of AMG-487 (Amgen, Thousand Oaks, CA). Briefly, mice were anesthetized with isoflurane (5\% inhalation, 2-4\% inhalation, continuous) and placed in a stereotaxic frame (Kopf, Tujunga, CA). AMG-487 was freshly prepared in artificial cerebral spinal fluid (ACSF) containing $10 \%$ of DMSO. $5 \mu \mathrm{L}$ of AMG-487 solution was gradually delivered to the right ventricle through a 26sgauge needle. The coordinates from bregma were anteroposterior $0.45 \mathrm{~mm}$, mediolateral $+1.0 \mathrm{~mm}$, and dorsoventral $-3.6 \mathrm{~mm}$. The needle remained in place for 30 seconds to minimize 
back-flux of the injectate. After 2 hours, animals were PIC-challenged and tested for sickness behavior, as in Chapter 2, 3.

\subsubsection{Microscopy}

Immunohistochemistry and confocal microscopy were performed as described in Chapter 3. Zstacks were taken using the 60X or $40 \mathrm{X}$ objective through the middle of Cornu Ammonis 1 (CA1) and layers $2 / 3$ of the cortex, approximately $1 \mathrm{~mm}$ from midline every $0.23 \mu \mathrm{m}$ at a resolution of $512 \times 512$ pixels. 3D projections were then rendered using NIS Elements Advanced Research imaging software (Nikon Instruments, Melville, NY)

Cross sectional 10X images were obtained with the Olympus VS120 Slide Scanner as described in Chapter 4.

To assess microglia proliferation, microglial cell bodies were identified as Iba1 positive perikarya containing DAPI positive nuclei. We used maximum intensity projections, and counted microglia blinded by hand using the Photoshop counter tool.

\subsubsection{Neuron-Microglia Surface Area Contact}

Neuronal surface area in direct contact with microglial processes was quantified using IMARIS (Bitplane Inc, Concord, MA). Briefly, 40X Z-stacks of the motor cortex, stained for neurons (NeuN) and microglia (lba1) were imported to IMARIS, and surface renderings for both neurons and microglia were created. A smoothing value of $0.6 \mu \mathrm{m}$ was utilized for neuronal cell bodies to eliminate dendritic and axonal arborization and background subtraction was applied. The minimum diameter of $5.00 \mu \mathrm{m}$ for neurons was determined by selecting the "slice view" and using the line tool to measure the diameter of the smallest neuronal cell body. A manual threshold of 40 was applied to all images, a value which was determined to best cover the cell body of neurons without extending into axonal segments or covering negative area. Seed points, to separate touching neurons, was applied at a quality above 40 with a threshold growing diameter of $9.00 \mu \mathrm{m}$. Finally, neuronal surfaces were required to have an area above $250 \mu \mathrm{m}^{2}$ to eliminate any partial neuronal surfaces or background fluorescence.

Microglial surfaces were rendered with a smoothing value of $0.4 \mu \mathrm{m}$, a value decided upon by selecting the "slice view" and using the line tool to measure the diameter of the average microglial processes. Background elimination was once again applied and the largest diameter to be remodeled was maximized at $1.00 \mu \mathrm{m}$, a value which ensured fully coverage of microglial 
cell bodies. A manual threshold of 50 was selected to ensure complete microglial process coverage in both control and PIC conditions. Finally, surfaces above 20 voxels and above the automatic threshold for the channel were selected to ensure removal of small microglial processes from non-visualized microglia as well as any background fluoresce.

The settings were applied to all samples and all neurons and microglia within the visual field of the z-stack. Once rendered, the surface-surface contact area X-Tension was applied to determine the percentage of neuronal surface area directly in contact with a microglial.

\subsubsection{Statistical Analysis}

Differences between groups were compared using Student's t- test and considered significant at $P \leq 0.05$.

\subsubsection{RESULTS AND DISCUSSION}

Throughout this study, we used the 24-hour post PIC challenge, because neuronal hyperexcitability peaks at this time point (Kirschman et al., 2011; Hunsberger et al., 2016). Microglia activation was observed throughout the brain following induction of peripheral PIC challenge. Fig. 1 shows representative images of Iba1 staining of the hippocampal CA1 subregion and the motor cortex. In both regions, PIC challenge lead to a profound hypertrophy of microglia and retraction of their processes. 
A

CA1
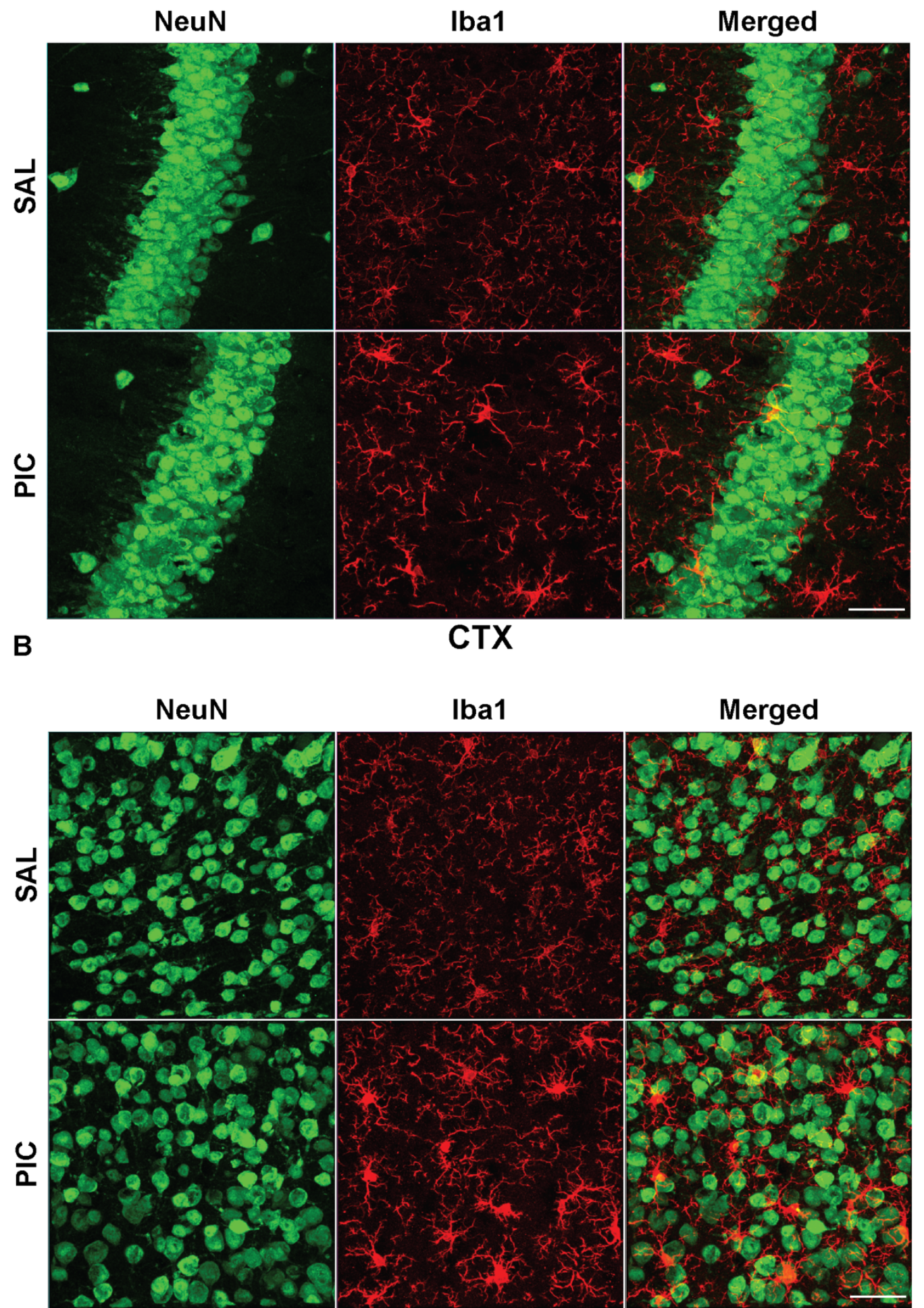

Figure 1. PIC challenge induces microglial activation. Mice were i.p. injected PIC or saline (SAL) were analyzed by immunohistochemistry after 24 hours. Microglial expression (red) costained with NeuN (green) in the CA1 subregion of the hippocampus [A] and cortex [B]. Confocal images were captured at 60X magnification. Scale bars represent $50 \mu \mathrm{m}$. 
As activated microglia often proliferate in response to stimuli, we counted the number of microglial cell bodies within the visual field $\left(101.25 \mathrm{~mm}^{2}\right.$ area) of the CA1, CA3, and DG at 24 hours after saline (SAL) or PIC injection. No difference in the number of cell bodies was observed in the CA1, CA3, or DG subregions of the hippocampus No difference in the number of cell bodies was observed within the CA1 [SAL=9.5 $\pm 0.80, \mathrm{PIC}=10.0 \pm 0.81 ; \mathrm{t}(24)=-0.437$, $\mathrm{p}=0.666$ ], the CA3 [SAL=7.5 $\pm 0.59, \mathrm{PIC}=8.4 \pm 0.87 ; \mathrm{t}(22)=-0.888, \mathrm{p}=0.384$ ] or the $\mathrm{DG}$ $[S A L=9.67, P I C=11.42 ; t(22)=-1.207, p=0.240]$, indicating that despite microglial activation at 24 hours, microglia are not proliferating.

As microglia can interact with axo-somatic synapses to modulate neuronal activity, we determine the covering of neuronal cell bodies by microglial processes in PIC challenged vs. control brains using the IMARIS system. The accuracy of IMARIS modeling of neurons and microglia, as well as the representative results of surface-surface contact on each surface is depicted in Fig. 2. PIC challenge seemed to increase the percentage of cortical neuronal surface covered by microglial by approximately $30 \%$. However, the difference was not statistically significant between saline treated $(5.39 \pm 1.90)$ and PIC challenged animals $(7.93 \pm 0.85)[t(7)=1.442, p=0.193]$. Moreover, no difference in either the number of neuronal perikarya or neuronal surface area was evident (data not shown). 
A

SAL

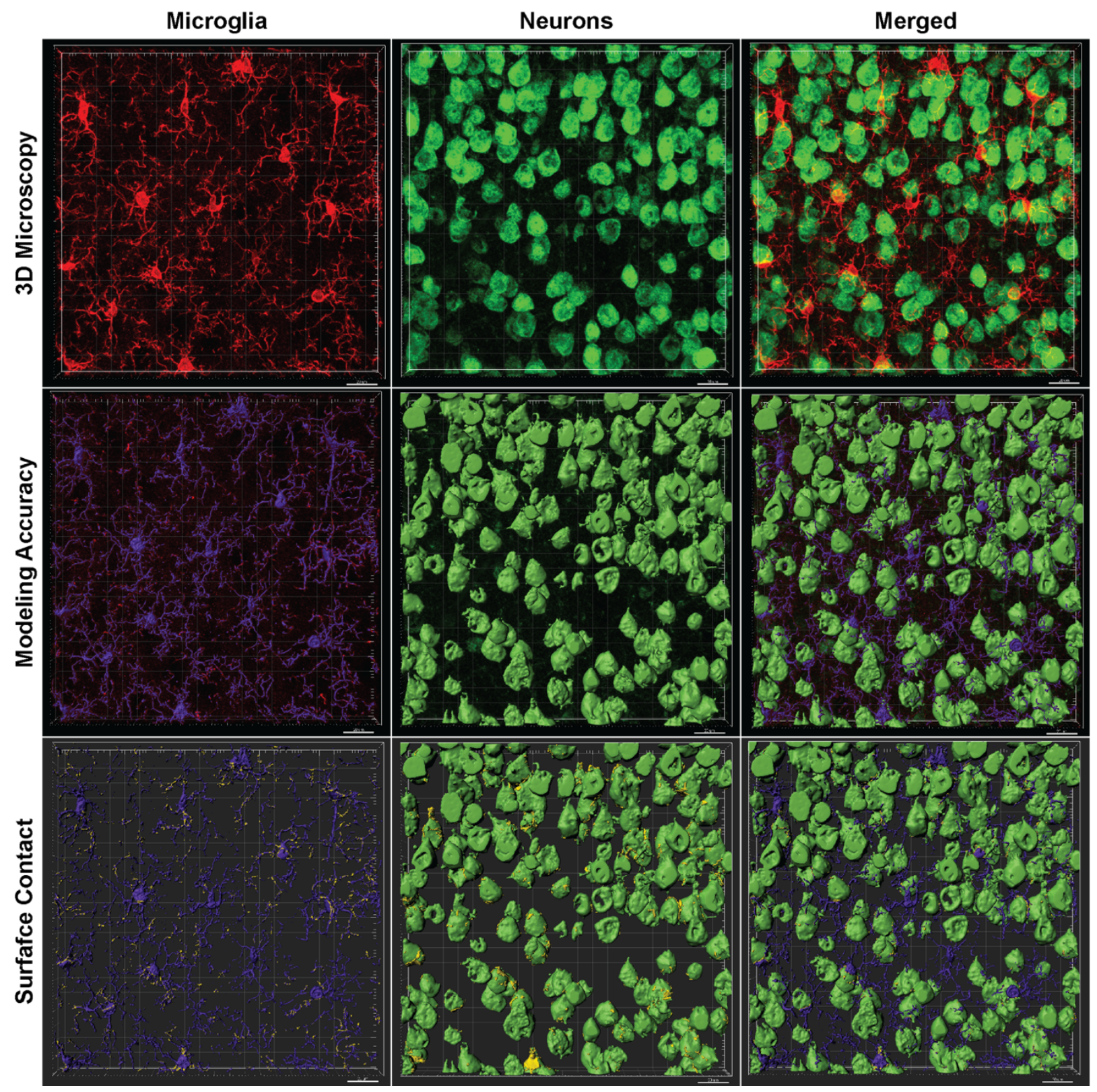




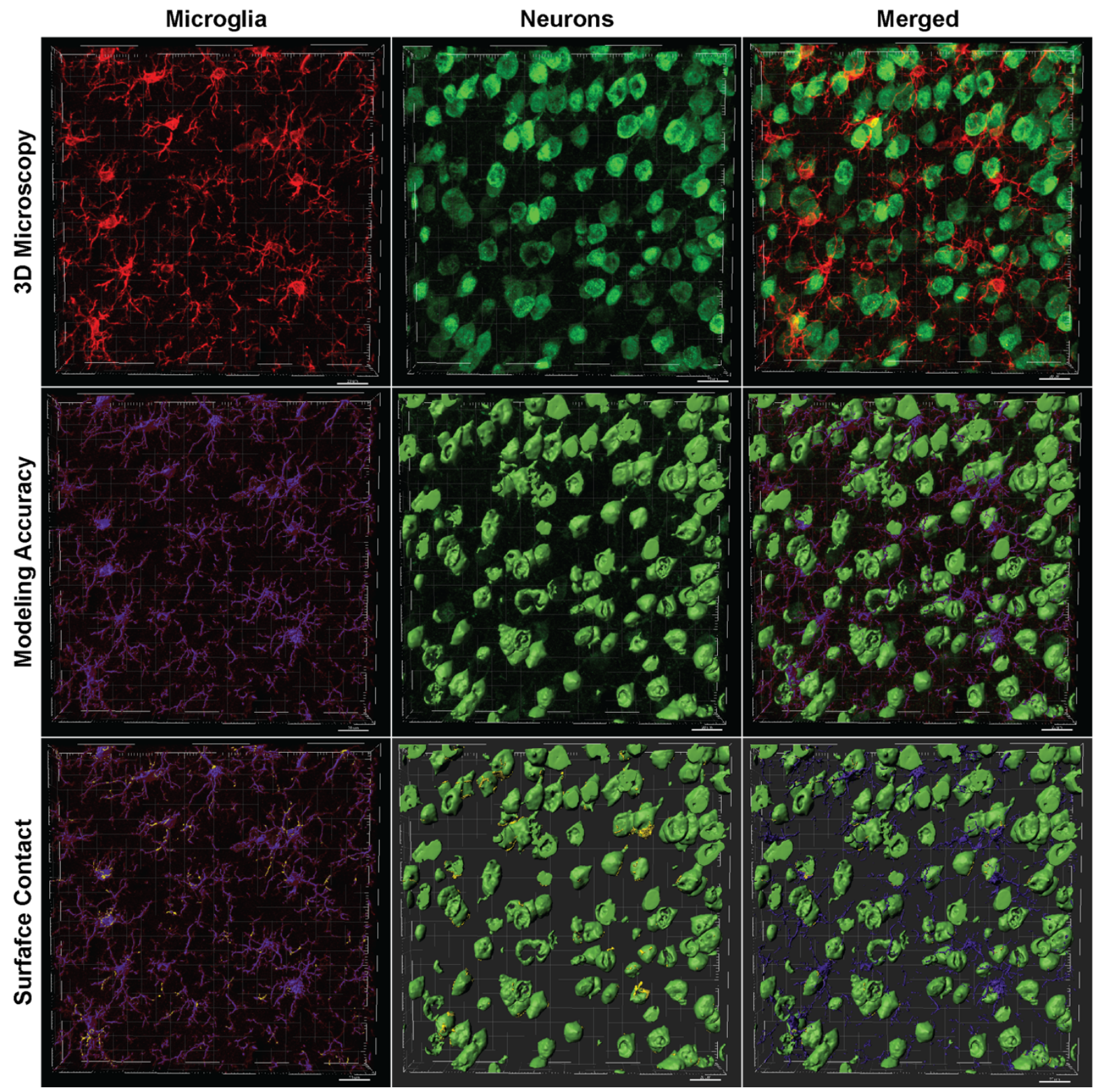

Figure 2. IMARIS modeling of neuron-microglia interactions. Mice were injected with saline $[A]$ or PIC $[B]$ and the cortices were analyzed by immunohistochemistry 24 hours later. Microglia were stained red and neurons were stained green. Confocal images were captured at 40X magnification. Images were imported into IMARIS, and the surface models for glia and neurons were created (see Methods). Top panels represent original microscopy images in 3D. Middle panels demonstrate the accuracy of the neuron and glial models. Lower panels highlight the contacts (in yellow) between neurons and microglia. Scale bars represent $20 \mu \mathrm{m}$. 


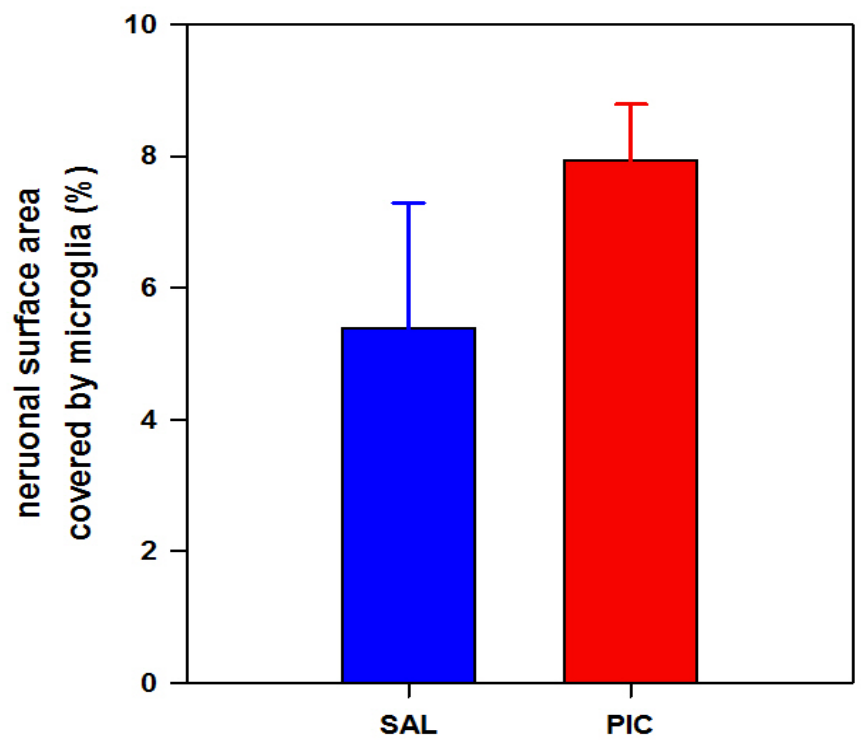

Figure 3. Interaction of microglia with cortical neurons following PIC challenge. After rendering neuron and microglia models, the surface-surface contact area $X$-tension was applied to determine the percentage of total neuronal surface directly in contact with microglia. (Figure 2). Data are presented as means \pm SEM from 3-4 cortical images (approximate volume of each image was $1518.75 \mathrm{~mm}^{3}$ ). The difference between SAL and PIC groups was assessed by Student's t-test. $p=0.193$.

These results indicate that in response to PIC challenge, microglia undergo activation that entails hypertrophy and retraction of processes, but not proliferation. Moreover, this activation does not involve increased neuronal contacts. Nevertheless, it remains plausible that microglia are affecting neuronal synapses. For example, microglia may secrete factors that disrupt the extracellular matrix (Chen et al., 2014; Akiyoshi et al., 2018), or factors that directly alter synaptic activity (Beattie et al., 2002; Ji et al., 2013).

However, signals that activate microglia are not known. CXCL10, which is robustly produced in response to PIC challenge (Chapters 2 and 3), is a plausible candidate. However, microglia do not express its receptor, CXCR3 (Chapter 3). Therefore, we consider three possible mechanisms. First, microglial activation is elicited indirectly by factors release from other cells in response to CXCR3 ligation with CXCL10. Second, microglial activation is mediated by TLR4 signaling, because CXCL10 can efficiently activate TLR4 (Schulthess et al., 2009) and microglia strongly express TLR4 (Olson and Miller, 2004). Third, the minor chemokines generated by PIC challenge, e.g., CXCL1 or CXCL2 (Chapter 2), mediate microglial activation (Fan et al., 2017)

To address these mechanisms, we blocked cerebral CXCR3 using i.c.v. injection of AMG-487, the non-competitive inhibitor of the receptor. Because the inhibitor was delivered in 10\% DMSO, respective controls were i.c.v. injected with DMSO solution alone. Similarly, mice i.p. injected with saline served as controls for PIC challenged mice. 
As shown in Figure 4, microglia from mice injected with DMSO + SAL displayed a resting, ramified morphology that was identical to SAL-injected mice (Fig. 1). In mice injected with DMSO + PIC, microglia were robustly activated, as evidenced by their hypertrophy and retraction of fine processes. Blockage of CXCR3 attenuated microglial activation. The injection of AMG-487 alone induced microglia activation, but to a lesser extent than PIC challenge.

Similar results were observed throughout all brain regions (not shown).

Based on these results, we postulate the indirect mechanism of microglial activation.

Accordingly, CXCL10 binds to neuronal CXCR3 leading to the release of secondary factors.

These factors, in turn, elicit microglial activation.
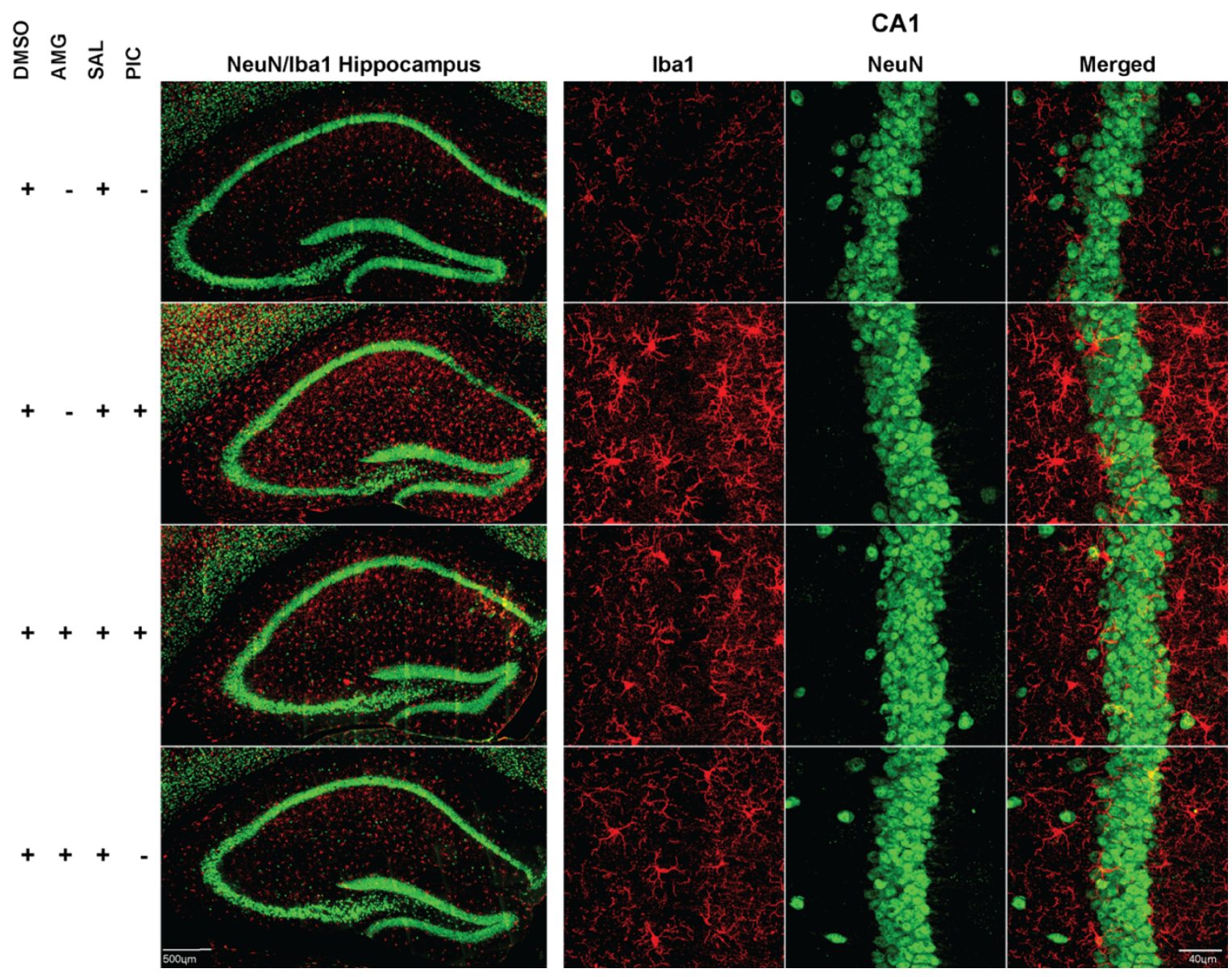

Figure 4. CXCR3 Inhibition attenuates microglia activation induced by PIC. Mice were i.c.v injected with AMG-487 or DMSO. Two hours later, mice were i.p. injected with PIC or saline (see Methods). Twenty-four hours after PIC injection brains were analyzed by immunohistochemistry using anti-lba1 antibody for microglia (red) and anti-NeuN antibody for neurons (green). Left panel shows 20X epiflorescent images of the hippocampus. Right panel shows $40 X$ confocal images of the CA1 subregion of the hippocampus. 


\subsection{IN VITRO EFFECT OF PIC CHALLENGE ON GLUTAMATE UPTAKE}

\subsubsection{INTRODUCTION}

We have previously shown that PIC challenge-induced hyperexcitability of hippocampal neuronal networks is concomitant with a robust increase in the level of extracellular glutamate in the hippocampus (Hunsberger et al., 2016). This is important finding because increased extracellular glutamate leads to neuronal hyperexcitability (Campbell and Hablitz, 2004, 2008; Featherstone and Shippy, 2008; Campbell et al., 2012; Campbell et al., 2014; Putatunda et al., 2014), and thus, may reveal molecular mechanisms by which PIC challenge enhances excitatory transmission. We have also shown that hippocampi of PIC-challenged mice had impaired uptake of glutamate that might underscore the increased extracellular glutamate, and thus, neuronal hyperexcitability. However, mechanistic studies of glutamate uptake in living animals are laborious, timely, and require a great number of animals. Thus, the present study was undertaken to test whether glutamate uptake can be analyzed in an in vitro model system of hippocampal slices to increase the efficiency of such studies.

\subsubsection{METHODS}

\subsubsection{Preparation of hippocampal slices}

C57BL/6 J mice were PIC-challenged as previously described (Chapters 2) and analyzed $24 \mathrm{hr}$ later. Mice were anesthetized with isoflurane, and brains were removed and immediately placed in ice-cold phosphate buffered saline (PBS). Hippocampi were dissected and cut into $350 \mu \mathrm{m}$ slices using a Mcllwain Tissue Chopper (Campden Instruments Ltd., Layfette, IN). The slices were pre-incubated for 30 minutes at room temperature in an incubation chamber containing circulating artificial cerebral spinal fluid (ACSF). ACSF consisted of $124 \mathrm{mM} \mathrm{NaCl}, 26 \mathrm{mM}$, $\mathrm{NaHCO}_{3}, 4.4 \mathrm{mM} \mathrm{KCl}, 1 \mathrm{mM}$ of $\mathrm{NaH}_{2} \mathrm{PO}_{4}, 1.3 \mathrm{mM}$ of $\mathrm{MgSO}_{4}, 2.5 \mathrm{mM} \mathrm{CaCl}, 10 \mathrm{mM}$ glucose, and was saturated with $5 \% \mathrm{CO}_{2} / 95 \% \mathrm{O}_{2}$.

\subsubsection{2. $\left[{ }^{3} \mathrm{H}\right]$-glutamate uptake}

Intracellular uptake of glutamate was determined as described by Griffin et al. (2015). Briefly, following pre-incubation, hippocampal slices were transferred into glass vials, and incubated in ACSF containing $250 \mathrm{nM}\left[{ }^{3} \mathrm{H}\right]$-glutamate $(51 \mathrm{Ci} / \mathrm{mM}$; Perkin Elmer, Waltham, MA) and $100 \mu \mathrm{M}$ unlabeled glutamate. The slices were incubated for seven min at $37^{\circ} \mathrm{C}$ with shaking. The uptake of glutamate was stopped by placing the vials on ice and aspirating the incubation medium. 
Slices were washed five times with ice-cold saline containing $100 \mu \mathrm{M}$ glutamate. The slices were solubilized with $5 \%$ SDS overnight at room temperature followed by the addition of the scintillation cocktail Ultima Gold (Perkin Elmer). The radioactivity was determined in the LS 6500 Multi-Purpose scintillation counter (Beckman Coulter, Brea, CA), and expressed as counts per minute (CPM).

\subsubsection{Protein Determination}

Total protein was measured in SDS-solubilized homogenates by ABC method (BioRad, Hercules, CA) using bovine serum albumin as a standard. The protein content was used to normalize the radioactivity to CPM per ug of protein.

\subsubsection{Statistical Analysis}

Differences between groups were compared using Student's t- test and considered significant at $P \leq 0.05$.

\subsubsection{RESULTS AND DISCUSSION}

In concordance to previous study in slices of the nucleus accumbens (Griffin et al., 2015), hippocampal slices avidly incorporated $\left[{ }^{3} \mathrm{H}\right]$-glutamate (Fig. 1). The incorporation in slices from saline-injected and PIC-injected mice averaged 844 and 969 CPM/ug of protein, respectively. The difference between these two values was non-significant $[t(18)=1.310, p=0.207]$.

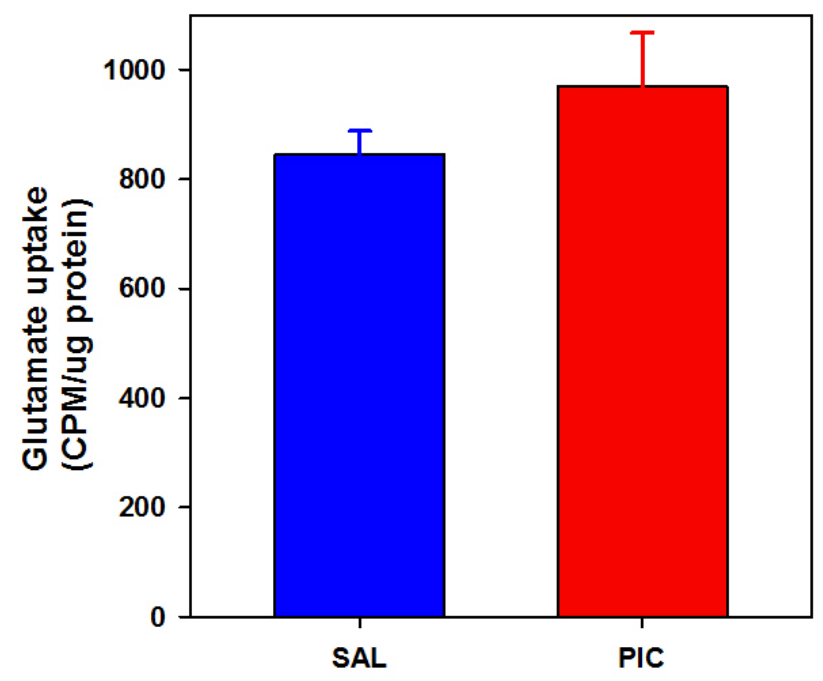

Figure 1. Hippocampal glutamate uptake following PIC challenge. Mice were i.p. injected with $12 \mathrm{mg} / \mathrm{kg}$ PIC or equivolume saline. After 24 hours, the uptake of [ $\left.{ }^{3} \mathrm{H}\right]-$ glutamate was determined in hippocampal slices and expressed as CPM/ug of protein. Bars represent averages \pm SDs from 5 animals.

In contrast to our previous in vivo study revealing a robust (approximately 4-8-fold) impairment of exogenous glutamate uptake instigated by PIC challenge (Hunsberger et al 2016), no 
impairment of glutamate uptake was evident between hippocampal slices from saline-injected vs. PIC-injected mice. This discrepancy is likely due to the artifacts of the in vitro system. For instance, our transcriptome analysis (Michalovicz, 2015) revealed no change in the expression of the message encoding the excitatory amino acid transporter 2 (EAAT2), the astrocytic protein responsible for $\sim 90 \%$ of extracellular glutamate uptake in the brain (Kim et al., 2011). Therefore, the impairment of glutamate uptake is likely due to a modification of EAAT2 activity. For example, a binding of a protein or other factors that regulate EAAT2 activity in vivo can be envisaged. These regulatory factors might have been lost in our in vitro system.

In conclusion, the hippocampal slice system is not applicable for studies of alterations of glutamate uptake instigated by PIC challenge. Such studies must be performed in vivo, using EAAT2 inhibitors (e.g., TBOA), activators (e.g., sulbactam), and/or transgenic techniques (e.g., knock-in and knock-out). 


\subsection{REFERENCES}

Akiyoshi R, Wake H, Kato D, Horiuchi H, Ono R, Ikegami A, Haruwaka K, Omori T, Tachibana Y, Moorhouse AJ, Nabekura J (2018) Microglia Enhance Synapse Activity to Promote Local Network Synchronization. eNeuro 5.

Aldskogius H, Liu L, Svensson M (1999) Glial responses to synaptic damage and plasticity. J Neurosci Res 58:33-41.

Baille-Le Crom V, Collombet JM, Burckhart MF, Foquin A, Pernot-Marino I, Rondouin G, Lallement G (1996) Time course and regional expression of C-FOS and HSP70 in hippocampus and piriform cortex following soman-induced seizures. J Neurosci Res 45:513-524.

Barros VN, Mundim M, Galindo LT, Bittencourt S, Porcionatto M, Mello LE (2015) The pattern of c-Fos expression and its refractory period in the brain of rats and monkeys. Front Cell Neurosci 9:72.

Beattie EC, Stellwagen D, Morishita W, Bresnahan JC, Ha BK, Von Zastrow M, Beattie MS, Malenka RC (2002) Control of synaptic strength by glial TNFalpha. Science 295:22822285.

Bullitt E (1990) Expression of c-fos-like protein as a marker for neuronal activity following noxious stimulation in the rat. J Comp Neurol 296:517-530.

Campbell SL, Hablitz JJ (2004) Glutamate transporters regulate excitability in local networks in rat neocortex. Neuroscience 127:625-635.

Campbell SL, Hablitz JJ (2008) Decreased glutamate transport enhances excitability in a rat model of cortical dysplasia. Neurobiol Dis 32:254-261.

Campbell SL, Buckingham SC, Sontheimer H (2012) Human glioma cells induce hyperexcitability in cortical networks. Epilepsia 53:1360-1370.

Campbell SL, Hablitz JJ, Olsen ML (2014) Functional changes in glutamate transporters and astrocyte biophysical properties in a rodent model of focal cortical dysplasia. Front Cell Neurosci 8:425.

Chen Z, Jalabi W, Hu W, Park HJ, Gale JT, Kidd GJ, Bernatowicz R, Gossman ZC, Chen JT, Dutta R, Trapp BD (2014) Microglial displacement of inhibitory synapses provides neuroprotection in the adult brain. Nat Commun 5:4486.

Cunningham C (2013) Microglia and neurodegeneration: the role of systemic inflammation. Glia 61:71-90.

Cunningham CL, Martinez-Cerdeno V, Noctor SC (2013) Microglia regulate the number of neural precursor cells in the developing cerebral cortex. J Neurosci 33:4216-4233.

Dudek FE (2006) Activation of Fos during spontaneous hippocampal seizures in a model of temporal lobe epilepsy. Epilepsy Curr 6:57-58.

Fan Y, Xie L, Chung CY (2017) Signaling Pathways Controlling Microglia Chemotaxis. Mol Cells 40:163-168.

Featherstone DE, Shippy SA (2008) Regulation of synaptic transmission by ambient extracellular glutamate. Neuroscientist 14:171-181.

Fields RD, Eshete F, Stevens B, Itoh K (1997) Action potential-dependent regulation of gene expression: temporal specificity in ca2+, cAMP-responsive element binding proteins, and mitogen-activated protein kinase signaling. J Neurosci 17:7252-7266.

Franco R, Fernandez-Suarez D (2015) Alternatively activated microglia and macrophages in the central nervous system. Prog Neurobiol 131:65-86.

Griffin WC, Ramachandra VS, Knackstedt LA, Becker HC (2015) Repeated cycles of chronic intermittent ethanol exposure increases basal glutamate in the nucleus accumbens of mice without affecting glutamate transport. Front Pharmacol 6:27.

Herrera DG, Robertson HA (1996) Activation of c-fos in the brain. Prog Neurobiol 50:83-107. 
Hong S, Dissing-Olesen L, Stevens B (2016) New insights on the role of microglia in synaptic pruning in health and disease. Curr Opin Neurobiol 36:128-134.

Hunsberger HC, Konat GW, Reed MN (2017) Peripheral viral challenge elevates extracellular glutamate in the hippocampus leading to seizure hypersusceptibility. J Neurochem 141:341-346.

Hunsberger HC, Wang D, Petrisko TJ, Alhowail A, Setti SE, Suppiramaniam V, Konat GW, Reed MN (2016) Peripherally restricted viral challenge elevates extracellular glutamate and enhances synaptic transmission in the hippocampus. J Neurochem 138:307-316.

Ji K, Miyauchi J, Tsirka SE (2013) Microglia: an active player in the regulation of synaptic activity. Neural Plast 2013:627325.

Joo JY, Schaukowitch K, Farbiak L, Kilaru G, Kim TK (2016) Corrigendum: Stimulus-specific combinatorial functionality of neuronal c-fos enhancers. Nat Neurosci 19:642.

Kawasaki M, Ponzio TA, Yue C, Fields RL, Gainer H (2009) Neurotransmitter regulation of c-fos and vasopressin gene expression in the rat supraoptic nucleus. Exp Neurol 219:212222.

Kawashima T, Okuno H, Bito H (2014) A new era for functional labeling of neurons: activitydependent promoters have come of age. Front Neural Circuits 8:37.

Kim K, Lee SG, Kegelman TP, Su ZZ, Das SK, Dash R, Dasgupta S, Barral PM, Hedvat M, Diaz P, Reed JC, Stebbins JL, Pellecchia M, Sarkar D, Fisher PB (2011) Role of excitatory amino acid transporter-2 (EAAT2) and glutamate in neurodegeneration: opportunities for developing novel therapeutics. J Cell Physiol 226:2484-2493.

Kirschman LT, Borysiewicz E, Fil D, Konat GW (2011) Peripheral immune challenge with dsRNA enhances kainic acid-induced status epilepticus. Metab Brain Dis 26:91-93.

Kovacs KJ (2008) Measurement of immediate-early gene activation- c-fos and beyond. J Neuroendocrinol 20:665-672.

Malhi SM, Jawed H, Hanif F, Ashraf N, Zubair F, Siddiqui BS, Begum S, Kabir N, Simjee SU (2014) Modulation of c-Fos and BDNF protein expression in pentylenetetrazole-kindled mice following the treatment with novel antiepileptic compound HHL-6. Biomed Res Int 2014:876712.

Michalovicz LT (2013) Peripheral challenge with a viral mimetic elicits seizure hypersusceptbilty In: Neuroscience. Graduate Theses, Dissertations, and Problem Reports: West Virginia University.

Michalovicz LT (2015) Peripheral challenge with a viral mimetic elicits seizure hypersusceptbility In. Graduate Theses, Dissertations, and Problem Reports West Viriginia University.

Morgan JI, Cohen DR, Hempstead JL, Curran T (1987) Mapping patterns of c-fos expression in the central nervous system after seizure. Science 237:192-197.

Morrison H, Young K, Qureshi M, Rowe RK, Lifshitz J (2017) Quantitative microglia analyses reveal diverse morphologic responses in the rat cortex after diffuse brain injury. Sci Rep 7:13211.

Nimmerjahn A, Kirchhoff F, Helmchen F (2005) Resting microglial cells are highly dynamic surveillants of brain parenchyma in vivo. Science 308:1314-1318.

Olson JK, Miller SD (2004) Microglia initiate central nervous system innate and adaptive immune responses through multiple TLRs. J Immunol 173:3916-3924.

Putatunda R, Hala TJ, Chin J, Lepore AC (2014) Chronic at-level thermal hyperalgesia following rat cervical contusion spinal cord injury is accompanied by neuronal and astrocyte activation and loss of the astrocyte glutamate transporter, GLT1, in superficial dorsal horn. Brain Res 1581:64-79.

Schafer DP, Lehrman EK, Stevens B (2013) The "quad-partite" synapse: microglia-synapse interactions in the developing and mature CNS. Glia 61:24-36. 
Schulthess FT, Paroni F, Sauter NS, Shu L, Ribaux P, Haataja L, Strieter RM, Oberholzer J, King CC, Maedler K (2009) CXCL10 impairs beta cell function and viability in diabetes through TLR4 signaling. Cell Metab 9:125-139.

Sheng M, McFadden G, Greenberg ME (1990) Membrane depolarization and calcium induce cfos transcription via phosphorylation of transcription factor CREB. Neuron 4:571-582.

Shevelkin AV, Efimova OI, Nikitin VP, Anokhin KV, Sherstnev VV (2012) Specific changes in cfos expression and colocalization with DNA in identified neuronal nuclei of edible snail following neurotransmitter application. Bull Exp Biol Med 153:734-737.

Srinivasan D, Yen JH, Joseph DJ, Friedman W (2004) Cell type-specific interleukin-1beta signaling in the CNS. J Neurosci 24:6482-6488.

Thompson MA, Ginty DD, Bonni A, Greenberg ME (1995) L-type voltage-sensitive Ca2+ channel activation regulates c-fos transcription at multiple levels. J Biol Chem 270:42244235.

Zhang J, Zhang D, McQuade JS, Behbehani M, Tsien JZ, Xu M (2002) c-fos regulates neuronal excitability and survival. Nat Genet 30:416-420. 


\section{CHAPTER 5: PERIPHERAL VIRAL CHALLENGE EXACERBATES EXPERIMENTAL AUTOIMMUNE ENCEPHALOMYELITIS}

Original Article: Metabolic Brain Disease (2019) vol. 4 issue 2, pgs. 675-679

Tiffany J. Petrisko and Gregory W. Konat

Departments of Biochemistry and Neuroscience, Rockefeller Neuroscience Institute, West Virginia University School of Medicine, Morgantown, WV 26506, USA

\subsection{ABSTRACT}

Peripheral viral infections are potent triggers of exacerbation in multiple sclerosis (MS). Here, we used a preclinical model of MS, the experimental autoimmune encephalomyelitis (EAE) to corroborate this comorbidity in an experimental setting. EAE was induced by immunization of mice with MOG peptide, and paralysis was scored using a 5-point scale. At the onset of the chronic phase of the disease (Days 42-58 after MOG injection) the animals were divided into low responders (LR) and high responders (HR) with the mean score of 1.5 and 2.5, respectively. The acute phase response (APR) was induced by intraperitoneal injections of a viral mimetic, polyinosinic-polycytidylic acid (PIC). Two daily injections were performed on Days 42 and 44 ( $\mathrm{PIC}_{42,44}$ challenge) and on Days 54, 55 and 56 ( $\mathrm{PIC}_{54,55,56}$ challenge). $\mathrm{PIC}_{42,44}$ challenge had no effect of EAE disease, whereas PIC $_{54,55,56}$ challenge rapidly increased paralysis but only in $\mathrm{HR}$ group. This exacerbation ultimately led to animal death by Day 58 . These results demonstrate that antiviral APR is a potent exacerbator of EAE, and that this activity directly correlates with the severity of the disease. This in turn, indicates that antiviral APR might play a pivot role in linking peripheral viral infections with MS exacerbations.

\subsection{INTRODUCTION}

Multiple sclerosis (MS) is characterized by unpredicted occurrence of exacerbations that detrimentally affect the long-term disability. Epidemiological studies strongly implicate viral infections in the periphery as triggers of the exacerbations (Sibley et al., 1985; Andersen et al., 1993; Panitch, 1994; Edwards et al., 1998; Buljevac et al., 2002; Correale et al., 2006; Libbey and Fujinami, 2010). Importantly, viral infection-instigated exacerbations result in more persistent neural deteriorations than other exacerbations. Although the mechanisms have not been defined, several pathways have been suggested. For example, the infections may decrease the ability of the CNS to protect itself against autoimmune attack by leukocytes 
(Matullo et al., 2011), enhance the autoimmune responsiveness of the host leukocytes (Correale et al., 2006), or induce re-activation of a CNS-resident virus (Borkosky et al., 2012).

Because MS exacerbations are induced by various, and often unrelated viruses (Libbey and Fujinami, 2010; Kakalacheva et al., 2011), the triggering process is likely to involve a common viral signature. A good candidate is a double stranded RNA (dsRNA) that is generated by most viruses during their replication cycle (Jacobs and Langland, 1996; Weber et al., 2006). dsRNA is recognized by the major antiviral receptors of the host cells, i.e., toll-like receptor 3 (TLR3), retinoic acid-inducible gene 1 (RIG-1), melanoma differentiation-associated protein 5 (MDA-5) and protein kinase $R(P K R)$. The ligation of these receptors elicits the acute phase response (APR), the first line of antiviral defense characterized by the fulminant generation of interferons, cytokines, chemokines and other inflammatory mediators that curtail the spread of the infection. However, these inflammatory mediators are also conveyed via circulation to the CNS whereby they induce a "mirror inflammatory response" (Dantzer and Kelley, 2007). The inflammatory factors generated peripherally and/or centrally during antiviral APR are expected to exert a profound effect on the progression of MS.

A synthetic dsRNA, polyinosinic-polycytidylic acid (PIC), is a commonly used tool to induce antiviral APR [reviewed in (Hunsberger et al., 2016)]. APR induced by intraperitoneal PIC injection involves a robust but transient surge of blood cytokines, such as IL1 $\beta$, IL6, TNFa, CXCL9, CXCL10, CXCL1, CXCL2, CCL2, CCL7, CCL12 and IFNß (Michalovicz and Konat, 2014; Petrisko and Konat, 2017). The brain response to this surge entails the production of CXCL10, and to a lesser extent the production of CXCL1, CXCL2 and CXCL9. In addition, the cerebral response includes the production of complement proteins and the activation of anaphylatoxin cascades (Michalovicz and Konat, 2014; Petrisko and Konat, 2017). Most of these inflammatory factors have been implicated in the pathology of MS (Cheng and Chen, 2014; Pranzatelli, 2018).

We hypothesize that the inflammatory factors generated during antiviral APR augment the ongoing MS pathology resulting in the exacerbation of the disease. The present study was undertaken to test this hypothesis using the autoimmune model of MS, the experimental autoimmune encephalomyelitis (EAE) in mice. During the chronic phase of EAE, mice were challenged with $\mathrm{PIC}$, and progression of the disease was monitored and compared to that in EAE animals without PIC challenge. 


\subsection{MATERIALS AND METHODS}

\subsubsection{ANIMALS}

Ten-week old female C57BL/6 mice obtained from Charles River Laboratories (Wilmington, MA) were housed on Diamond Soft bedding under 12-h light/dark cycle with ad libitum access to food and water. All procedures were approved by the West Virginia University Animal Care and Use Committee and conducted in compliance with the guidelines published in the NIH Guide for the Care and Use of Laboratory Animals.

\subsubsection{EAE INDUCTION}

The Hooke Kits ${ }^{\mathrm{TM}}$ for EAE Induction (Hooke Laboratories, Lawrence, MA) was used according to the manufacturer's protocol. Briefly, on Day 0 , mice were lightly anesthetized with isoflurane and subcutaneously (s.c.) injected with the $\mathrm{MOG}_{35-55}$ emulsion in CFA into four sites of the back (50 $\mu \mathrm{L}$ per site), followed by an intraperitoneal (i.p.) injection of $300 \mathrm{ng}$ of pertussis toxin (PTx) in $100 \mu \mathrm{L}$ of saline. The PTx injection was repeated the next day (Day 1). The animals were scored daily for signs of EAE using a 5 point scale: 0 , unaffected; 1 , loss of tail tone; 2 , hind limb weakness; 3 , complete paralysis of hind limbs; 4 , quadriplegia; and 5 , moribund. If an animal appeared to be between two scores, an average score was assigned.

\subsubsection{PIC CHALLENGE}

On Day 42, mice were divided into high responders (HR) with scores $>2$ (mean score of $2.5 \pm 0.1$ ) and low responders $(L R)$ with scores $\leq 2$ (mean score of $1.5 \pm 0.1$ ). To induce antiviral APR, half of the animals in each group received two daily i.p. injections of $12 \mathrm{mg} / \mathrm{kg}$ of ultrapure PIC (Invivogen, San Diego, CA) in $100 \mu \mathrm{L}$ of saline. These injections were spaced two hours apart. The first PIC challenge was performed on Days 42 and 44 . The second PIC challenge was executed on Days 54, 55 and 56. The other half of the animals were injected with an equivolume saline in lieu of PIC. Thus, four groups were generated: LR-PIC, LR-Sal, HR-PIC and HR-Sal. The animals were scored and weighted daily. Severely affected mice were housed with animals of similar severity in cages containing HydroGel (Westbrook, ME). Animals that scored a 4.0 for two consecutive days or lost more than $30 \%$ of body weight were euthanized. A score of 5.0 was assigned to animals that were euthanized or died. 


\subsubsection{STATISTICAL ANALYSIS}

Statistical comparisons were performed by two-way repeated measures ANOVA (RMANOVA) followed by the Holm-Sidak post hoc test. A Kaplan-Meir plot was generated and the logrank test was used to test differences in survival time. Differences between groups were considered significant at $\mathrm{P} \leq 0.05$.

\subsection{RESULTS}

The initial wave of paralysis commenced on Day 10, peaked on Day 16 and partially resolved around Day 25 after MOG immunization (Fig. 1). This was followed by a second slower relapse that plateaued around Day 40 indicating a chronic phase of the disease. There was no mortality at any time point.

We tested the comorbidity of antiviral APR induced by PIC injection during the chronic EAE phase. Although the peak scores during the initial relapse (Fig. 1) were tightly clustered (e.g., $2.4 \pm 0.1$ at Day 16 ), the variability of sickness intensity increased substantially thereafter. Consequently, on Day 42, we divided the animals into two subpopulations based on the disease severity, i.e., high responder (HR) group with scores of $2.5 \pm 0.1$, and low responder (LR) group with scores of $1.5 \pm 0.1$. 
PIC is rapidly deactivated within the peritoneal cavity, and consequently a single PIC injection results in a bolus stimulation of the innate immune system (Hunsberger et al., 2016). Because viral replication, and thus dsRNA generation in the host are protracted, we performed two consecutive PIC injections two hours apart to better simulate APR instigated by viral infection. To assess the effect of PIC challenge severity, we used two paradigms. Initially, the animals were challenged with PIC on Days 42 and 44 ( $\mathrm{PIC}_{42,44}$ challenge). After ten days of recovery, the animals were injected for three consecutive days, i.e., Days 54, 55 and 56 ( $\mathrm{PIC}_{54,55,56}$ challenge).

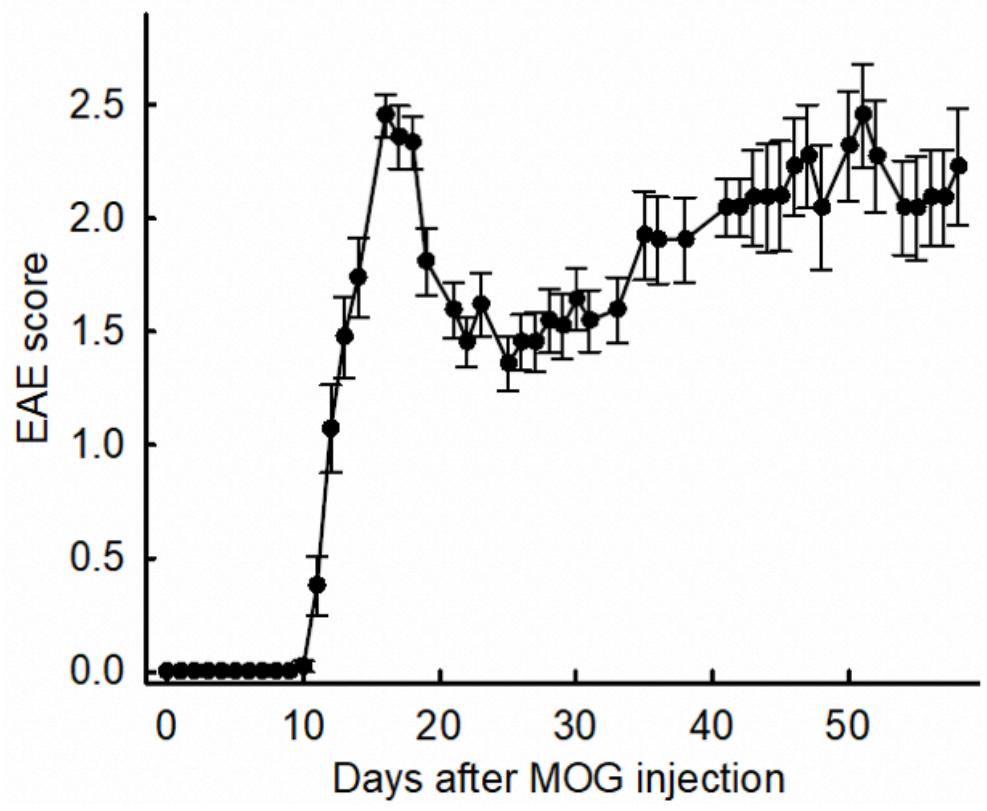

Figure 1. Time course of MOGinduced EAE. Ten-week old female C57BL/6 mice were immunized with $\mathrm{MOG}_{35-55}$ peptide (Day 0) and the progression of paralysis was scored daily (for details see Materials and Methods). Points represent averages \pm SE from 20 (Days 0 42) and 11 (Days 43-59) animals. 
The response of animals during the chronic phase of EAE to PIC challenge is shown in Fig. 2. RMANOVA on Days 42-59 showed a significant effect of group $[F(3,238)=21.48, p<0.001]$, and time $[F(14,238)=4.38, p<0.001]$. The interaction between groups and time was also significant $[F(42,238)=2.80, p<0.001]$. In $\mathrm{HR}$ group, $\mathrm{PIC}_{42,44}$ challenge tended to temporarily increase $\mathrm{EAE}$ scores as compared to EAE mice injected with saline, but the differences were not statistically significant. $\mathrm{PIC}_{54,55,56}$ challenge profoundly exacerbated paralysis, and the difference in $\mathrm{EAE}$ scores between HR-PIC and HR-Sal groups reached high statistical significance $(p<0.001)$ on Days 56, 57 and 58. Moreover, the exacerbation of EAE symptoms resulted in animal death. The first animal in HR-PIC group died on Day 56, and no animal survived beyond Day 58. The difference from HR-Sal group assessed by the logrank test was highly significant $(p \leq 0.001)$.
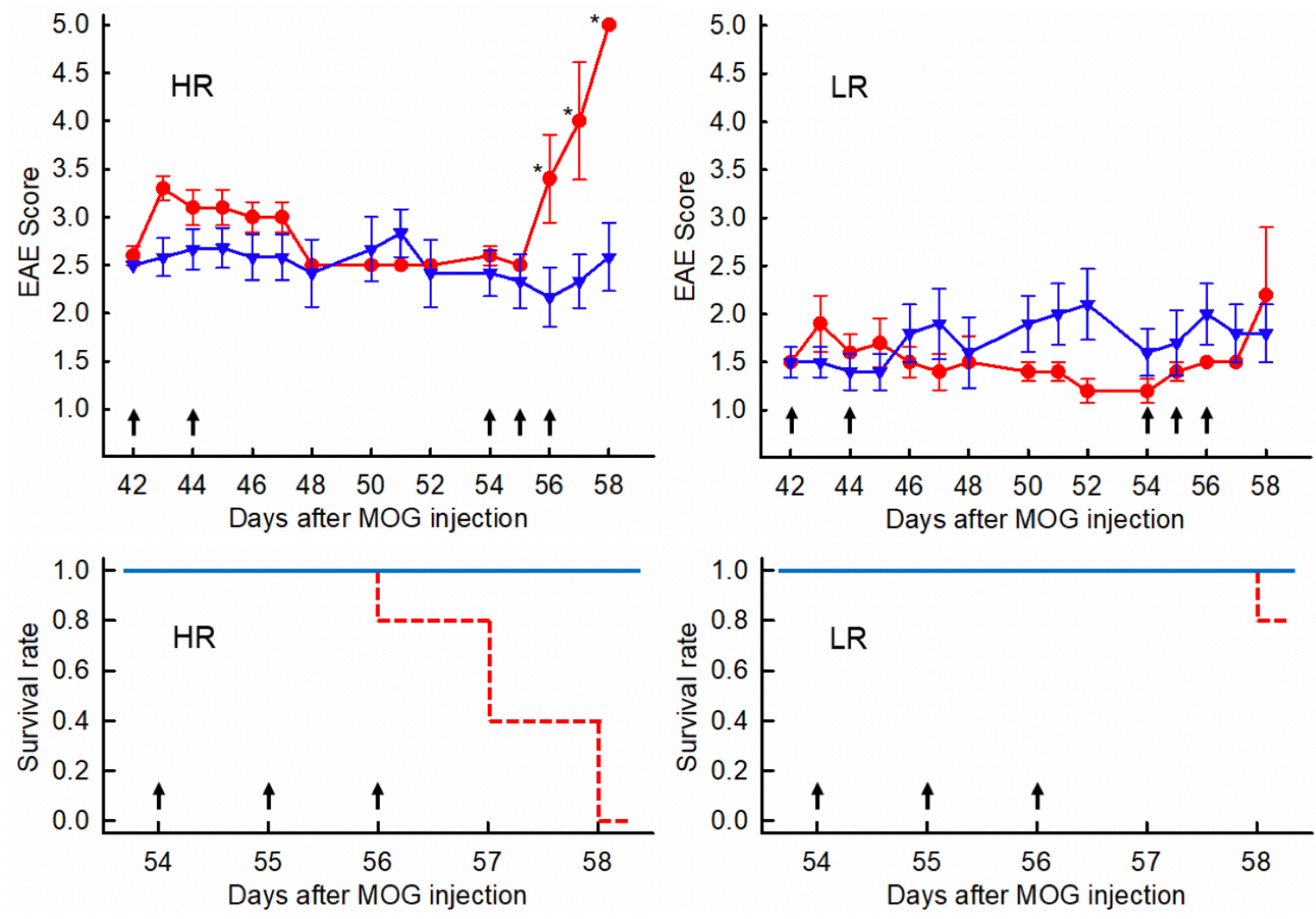

Figure 2. Comorbidity of PIC-induced APR during chronic phase of EAE. Upper panels show the effect of PIC challenges on neurological disability. Arrows indicate PIC injections during the first $\left(P C_{42,44}\right)$ and second $\left(P I C_{54,55,56}\right)$ challenge (for details see text). Red circles; $P I C$-injected EAE mice. Blue triangles; saline-injected EAE mice. Symbols represent averages \pm SE. LR-PIC (n=5), LR-Sal $(n=5)$, HR-PIC $(n=5)$ and HR-Sal $(n=6) .{ }^{*} P \leq 0.001$. Lower panels show mortality after the second PIC challenge. Brocken red lines represent PIC-challenged EAE mice. Solid blue lines represent saline-injected controls. 
In contrast, no change in sickness scores was observed in LR-PIC group as compared to LRSal group after either $\mathrm{PIC}_{42,44}$ or $\mathrm{PIC}_{54,55,56}$ challenges (Fig. 2). Although $\mathrm{PIC}_{42,44}$ challenge tended to ameliorate the disease in LR-PIC, the decrease of EAE scores was not statistically significant. Following PIC $_{54,55,56}$ challenge, one LR-PIC mouse died (20\% mortality) in on Day 56 , albeit the survival of this group did not significantly differ from LR-Sal group.

\subsection{DICUSSION}

Previous preclinical studies addressed the comorbidity of peripheral viral infections and MS by focusing on their involvement in the induction phase of EAE (Peacock et al., 2003; Verbeek et al., 2007). These experiments have revealed that preceding peripheral viral infections increase the susceptibility of animals to develop EAE. However, the effect of viral insult during the effector phase of EAE has not been studied. In the present study, we demonstrated comorbid effect of viral challenge in mice with established EAE. Consequently, our experimental paradigm models viral infection-induced exacerbations in MS patients (Sibley et al., 1985; Andersen et al., 1993; Panitch, 1994; Edwards et al., 1998; Buljevac et al., 2002; Correale et al., 2006; Libbey and Fujinami, 2010).

The effect of PIC-induced exacerbation of EAE disease depended on the strength of PIC challenge. Thus, no effect was evident in EAE mice following challenge on two alternate days $\left(\mathrm{PIC}_{42,44}\right)$. In contrast, PIC challenge for three consecutive days $\left(\mathrm{PIC}_{54,55,56}\right)$ induced a fulminant relapse of the disease as seen from the rapid exacerbation of paralysis and ultimate death. Although the animals were allowed to recover for ten days after the first PIC challenge, a possibility for a priming of the immune system for a heightened response to the second PIC challenge could also be considered.

The exacerbating effect of PIC challenge was also strongly dependent on the severity of EAE disease. Thus, the exacerbation of EAE symptoms occurred only in HR group, while no exacerbation was evident in LR group. It is expected that HR group with the initial score of 2.5 has more intense inflammation than LR group with the score of 1.5. These results might indicate the existence of a threshold in the inflammatory status required for the fulminant response to occur. Alternatively, the response might be more gradually dependent on disease severity. For example, although LR mice were evidently spared, a tendency to increase paralysis score and the death of one animals occurred on Day 58. Thus, the exacerbation in LR mice may be delayed beyond Day 58. This dependence on EAE severity is reminiscent of MS patient 
response to inflammatory mediators that is depend on the disease subtype (Nikfar et al., 2010). The effects of EAE severity as well as the strength of PIC challenge on EAE exacerbation will be delineated in future studies.

PIC is a potent inducer of generic APR in a manner independent of the viral type (GuhaThakurta and Majde, 1997; Traynor et al., 2004). The induction of antiviral APR is restricted to the peritoneal cavity, because PIC is swiftly degraded within this compartment, and does not reach the circulation (Konat, 2016). PIC stimulates peritoneal macrophages and mesothelial cells, but does not cause tissue damage, and thus does not elicit the confounding effects of systemic response to tissue injury. Consequently, the present study provides the proof-ofconcept that APR per se is a principal component of the innate immune response to viral challenge responsible for the exacerbation of $\mathrm{EAE}$, and by extension, for MS exacerbations.

The inflammatory factors generated during PIC-induced APR are likely responsible for the augmentation of EAE pathology. For example, IL6 that is profoundly increased by PIC challenge in the blood (Michalovicz and Konat, 2014) has been demonstrated to enhance the recruitment of patrolling leukocytes through the neuroendothelium (Richard et al., 2011). CXCL10 that is also robustly increased by PIC challenge in both the blood and CNS (Petrisko and Konat, 2017) is a potent activator and chemoattractant of circulating leukocytes (Liu et al., 2001) and resident microglia (Rappert et al., 2004; Clarner et al., 2015). The increase of leukocytic infiltration promoted by IL6 and/or CXCL10 is likely to augment the neuroinflammatory milieu in the CNS parenchyma leading to exacerbation of the disease. Moreover, PIC challenge upregulates the generation and activation of the complement proteins in the CNS (Michalovicz et al., 2015b; Petrisko and Konat, 2017), and the complement system activation is an intrinsic feature of MS pathology (Storch et al., 1998). PIC-induced APR may also act at the periphery by enhancing the activity of lymphocytes. For example, PIC is a potent promoter of expansion and differentiation of CD8+ T cells (Ngoi et al., 2008), which are primarily responsible for CNS damage during MS relapses (Malmestrom et al., 2008). Finally, one should also be cognizant of the possibility that EAE animals with ongoing autoimmune pathology may generate different profiles of inflammatory factors in response to PIC challenge that differ from those induced in naïve animals used in the aforementioned studies. The identification of these factors would open the way for the development of therapeutic strategies to control the progression of MS. 
In conclusion, this is the first study that translates the clinical correlation between peripheral viral infections and MS pathology to experimental exploration by focusing on the role of APR as the hub that links infections by unrelated viruses to MS exacerbations. We have developed a preclinical paradigm to examine this comorbidity, and demonstrated that antiviral APR exacerbates EAE in mice, an autoimmune model of MS. The PIC paradigm provides a unique model to identify the components of antiviral APR responsible for the disease exacerbation.

\section{Acknowledgements}

This study was supported by a Pilot Grant from the National Multiple Sclerosis Society awarded to G.W.K.

\section{Conflict of interest}

The authors declare no conflicts of interest. 


\subsection{REFERENCES}

Andersen O, Lygner PE, Bergstrom T, Andersson M, Vahlne A (1993) Viral infections trigger multiple sclerosis relapses: a prospective seroepidemiological study. J Neurol 240:417422.

Borkosky SS, Whitley C, Kopp-Schneider A, zur HH, de Villiers EM (2012) Epstein-Barr virus stimulates torque teno virus replication: a possible relationship to multiple sclerosis. PLoS ONE 7:e32160.

Buljevac D, Flach HZ, Hop WC, Hijdra D, Laman JD, Savelkoul HF, van Der Meche FG, van Doorn PA, Hintzen RQ (2002) Prospective study on the relationship between infections and multiple sclerosis exacerbations. Brain 125:952-960.

Cheng W, Chen G (2014) Chemokines and chemokine receptors in multiple sclerosis. Mediators of inflammation 2014:659206.

Clarner T, Janssen K, Nellessen L, Stangel M, Skripuletz T, Krauspe B, Hess FM, Denecke B, Beutner C, Linnartz-Gerlach B, Neumann H, Vallieres L, Amor S, OhI K, Tenbrock K, Beyer C, Kipp M (2015) CXCL10 triggers early microglial activation in the cuprizone model. J Immunol 194:3400-3413.

Correale J, Fiol M, Gilmore W (2006) The risk of relapses in multiple sclerosis during systemic infections. Neurology 67:652-659.

Dantzer R, Kelley KW (2007) Twenty years of research on cytokine-induced sickness behavior. Brain Behav Immun 21:153-160.

Edwards S, Zvartau M, Clarke H, Irving W, Blumhardt LD (1998) Clinical relapses and disease activity on magnetic resonance imaging associated with viral upper respiratory tract infections in multiple sclerosis. J Neurol Neurosurg Psychiatry 64:736-741.

Guha-Thakurta N, Majde JA (1997) Early induction of proinflammatory cytokine and type I interferon mRNAs following Newcastle disease virus, poly [rl:rC], or low-dose LPS challenge of the mouse. J Interferon Cytokine Res 17:197-204.

Jacobs BL, Langland JO (1996) When two strands are better than one: the mediators and modulators of the cellular responses to double-stranded RNA. Virology 219:339-349.

Kakalacheva K, Munz C, Lunemann JD (2011) Viral triggers of multiple sclerosis. Biochim Biophys Acta 1812:132-140.

Konat G (2016) Cerebral Response to Peripheral Challenge with a Viral Mimetic. Neurochem Res 41:144-155.

Libbey JE, Fujinami RS (2010) Potential triggers of MS. Results Probl Cell Differ 51:21-42.

Liu MT, Keirstead HS, Lane TE (2001) Neutralization of the chemokine CXCL10 reduces inflammatory cell invasion and demyelination and improves neurological function in a viral model of multiple sclerosis. J Immunol 167:4091-4097.

Malmestrom C, Lycke J, Haghighi S, Andersen O, Carlsson L, Wadenvik H, Olsson B (2008) Relapses in multiple sclerosis are associated with increased CD8+ T-cell mediated cytotoxicity in CSF. J Neuroimmunol 196:159-165.

Matullo CM, O'Regan KJ, Curtis M, Rall GF (2011) CNS recruitment of CD8+ T lymphocytes specific for a peripheral virus infection triggers neuropathogenesis during polymicrobial challenge. PLoS Pathog 7:e1002462.

Michalovicz LT, Konat GW (2014) Peripherally restricted acute phase response to a viral mimic alters hippocampal gene expression. Metab Brain Dis 29:75-86.

Michalovicz LT, Lally BE, Konat GW (2015) Peripheral challenge with a viral mimic upregulates expression of the complement genes in the hippocampus. J Neuroimmunol 285:137142.

Ngoi SM, Tovey MG, Vella AT (2008) Targeting poly(I:C) to the TLR3-independent pathway boosts effector CD8 T cell differentiation through IFN-alpha/beta. J Immunol 181:76707680 . 
Nikfar S, Rahimi R, Abdollahi M (2010) A meta-analysis of the efficacy and tolerability of interferon-beta in multiple sclerosis, overall and by drug and disease type. Clin Ther 32:1871-1888.

Panitch HS (1994) Influence of infection on exacerbations of multiple sclerosis. Ann Neurol 36 Suppl:S25-28.

Peacock JW, Elsawa SF, Petty CC, Hickey WF, Bost KL (2003) Exacerbation of experimental autoimmune encephalomyelitis in rodents infected with murine gammaherpesvirus- 68 . European journal of immunology 33:1849-1858.

Petrisko T, Konat G (2017) Peripheral viral challenge triggers hippocampal production of inflammatory proteins. Metab Brain Dis 32:1249-1254.

Pranzatelli MR (2018) Advances in Biomarker-Guided Therapy for Pediatric- and Adult-Onset Neuroinflammatory Disorders: Targeting Chemokines/Cytokines. Front Immunol 9:557.

Rappert A, Bechmann I, Pivneva T, Mahlo J, Biber K, Nolte C, Kovac AD, Gerard C, Boddeke HW, Nitsch R, Kettenmann H (2004) CXCR3-dependent microglial recruitment is essential for dendrite loss after brain lesion. J Neurosci 24:8500-8509.

Richard JF, Roy M, Audoy-Remus J, Tremblay P, Vallieres L (2011) Crawling phagocytes recruited in the brain vasculature after pertussis toxin exposure through IL6, ICAM1 and ITGalphaM. Brain Pathol 21:661-671.

Sibley WA, Bamford CR, Clark K (1985) Clinical viral infections and multiple sclerosis. Lancet 1:1313-1315.

Storch MK, Piddlesden S, Haltia M, livanainen M, Morgan P, Lassmann H (1998) Multiple sclerosis: in situ evidence for antibody- and complement-mediated demyelination. Annals of neurology 43:465-471.

Traynor TR, Majde JA, Bohnet SG, Krueger JM (2004) Intratracheal double-stranded RNA plus interferon-gamma: a model for analysis of the acute phase response to respiratory viral infections. Life Sci 74:2563-2576.

Verbeek R, van Dongen H, Wawrousek EF, Amor S, van Noort JM (2007) Induction of EAE by T cells specific for alpha B-crystallin depends on prior viral infection in the CNS. Int Immunol 19:277-285.

Weber F, Wagner V, Rasmussen SB, Hartmann R, Paludan SR (2006) Double-stranded RNA is produced by positive-strand RNA viruses and DNA viruses but not in detectable amounts by negative-strand RNA viruses. J Virol 80:5059-5064. 


\section{CHAPTER 6: DICUSSION}

This dissertation research is based on and expands previous study from our laboratory by addressing molecular mechanisms, by which peripheral PIC challenge induces neuronal hyperexcitability. In this chapter, we discuss the major findings and their impact on elucidating these mechanisms.

\subsection{CXCL10/CXCR3 AXIS}

The primary discovery of this research is that cerebral CXCL10/CXCR3 axis mediates the development of neuronal hyperexcitability in response to anti-viral APR. This axis is an important component of pathological processes. For example, CXCL10 is a chemoattractant responsible for the migration of leukocytes to the site of infection, and polarizes $T$ cells towards a proinflammatory, Th1/Th17 phenotype via ligation to CXCR3 (Dufour et al., 2002; Groom and Luster, 2011b; Kariya et al., 2016). CXCL10 can either inhibit or promote proliferation of endothelial cells, depending on the subtype of CXCR3 (Bodnar et al., 2006; Hueso et al., 2018). CXCL10 is also a key mediator of several peripheral immune conditions, including psoriasis, Chron's disease, and rheumatoid arthritis (Berry, 2004; Lee et al., 2017). The importance of the CXCL10/CXCR3 axis has led to the development of targeted therapeutic strategies. Thus, numerous anti-CXCL10 antibodies have shown success in phase II clinical trials (Angiolillo et al., 1995; Wijtmans et al., 2008; Yellin et al., 2012; Mayer et al., 2014), indicating therapeutic effect of reducing CXCL10 signaling. Also, a plethora of CXCR3 inhibitors have been developed (Andrews and Cox, 2016), and made their way to clinical trials (Floren LC, 2003; Berry, 2004) (Fig. 1). 


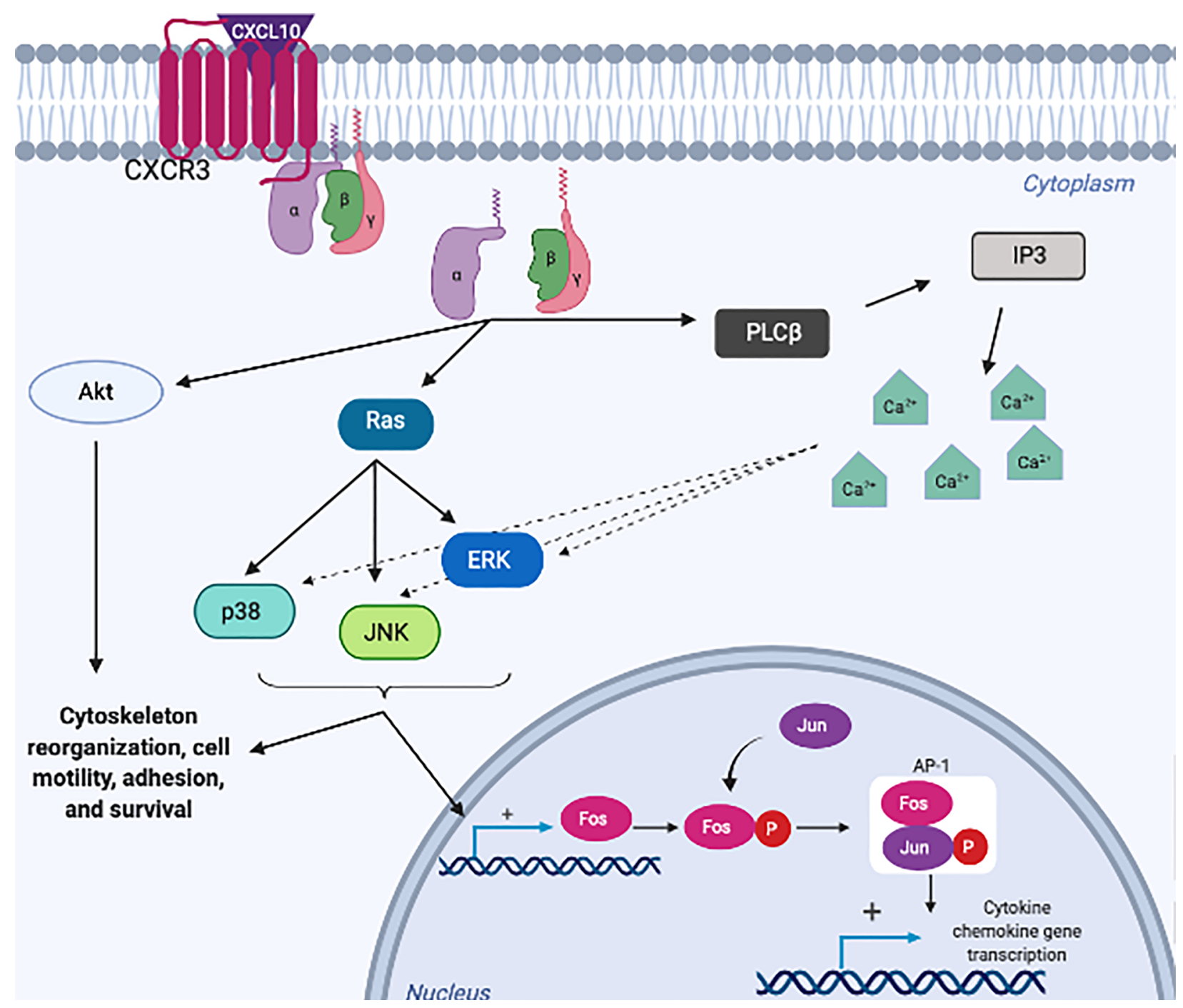

Figure 1. Singling pathways activated following neuronal CXCR3 activation. Ligation of CXCL10 to $C X C R 3$ results in dissociation of $\mathrm{G} \alpha$ from the catalytic $\mathrm{G} \beta \gamma$ subunit. In turn, these subunits activate multiple pathways, including Akt, Ras, and PLC $\beta$, with the latter resulting in increases of intracellular calcium. Additionally, activation of the Ras pathway promotes production of c-fos. As a transcription factor itself, c-fos along with c-Jun and AP-1 result in the production of additional cytokines and chemokines. Adapted from Dabiri et al. (2016)

While the specific role of the CXCL10/CXCR3 axis in brain pathology is not yet understood, many studies have established a negative impact of increased CXCL10 and CXCR3 expression on the progression of neurological diseases (Sorensen et al., 2002; Klein, 2004; Michlmayr and McKimmie, 2014a; Vazirinejad et al., 2014; Krauthausen et al., 2015; Roberts et al., 2015; Iwanowski et al., 2017). For example, elevated CXCL10 and CXCR3 levels have been demonstrated in the cerebrospinal fluid (CSF) of MS patients, and these elevations are 
correlated with clinical relapses. CXCL10 is produced in active demyelinating lesions by macrophages and astrocytes, and likely contributes to the tissue distraction. Alzheimer's disease (AD) patients also exhibit increased levels of CXCL10 and CXCR3, and animal models have implicated the CXCR3 signaling in the induction of plaque formation and the promotion of cognitive and behavioral deficits.

Genetic knockout models have been created for both CXCL10 and CXCR3 and applied to neuroinflammatory paradigms. For example, CXCL10-/- mice infected with West Nile virus (WNV) have increased viral burden within the brain as well as increased mortality due to the inability of infected neurons to properly recruit peripheral immune cells (Klein et al., 2005). In experimental autoimmune encephalomyelitis (EAE), astrocytic $\mathrm{CXCL} 10^{-/}$mice demonstrated delayed onset of experimental autoimmune encephalomyelitis, but did not prevent progressive axonal loss (Mills Ko et al., 2014) while global deletion of CXCR3 worsened disease outcome in EAE mice (Müller et al., 2007). However, caution must be used when interpreting the results of knockout studies as C57BL/6 mice, which were utilized throughout the above studies. As mentioned before, C57BL/6 mice are deficient for CXCL11, which also binds to CXCR3, due to a point mutation. As CXCL10 deficient mice were generated using 129 embryonic stem cells that express the wild-type CXCL11, these mice as regained a functioning CXCL11 protein and compensatory mechanisms of this protein cannot be discounted (Groom and Luster, 2011a). Additionally, CXCR3 deficient mice have a deficiency of natural killer cells suggesting a regulatory role of CXCR3 (Christensen et al., 2004).Additionally, the neuroinflammatory conditions studied with CXCL10/CXCR3 knockout mice exhibit profound breakdown of the blood-brain barrier (BBB) and the severity of the disease model is mediated by recruitment of peripheral immune cells to the CNS, while our model does not exhibit breakdown of the BBB (unpublished data) nor the recruitment of peripheral immune cells (confirmed by lack of ameboid macrophage morphology in Iba1 or Cd11b stained glia). As such, an non-neuronal process, such as T cell recruitment or generation of effector cells could impact what appears to be a CNS mediated effect (Michlmayr and McKimmie, 2014b).

On the other hand, these (Andersen et al., 1993; Murray et al., 1993; Panitch, 1994; George et al., 1997; Edwards et al., 1998; Nee and Lippa, 1999; Buljevac et al., 2002; Holmes et al., 2003; Libbey and Fujinami, 2010; Holmes, 2013), and other neuropathological conditions, i.e., epilepsy (Tellez-Zenteno et al., 2005; Vezzani and Granata, 2005; Verrotti et al., 2009), Parkinson's disease (Zheng et al., 2012; Brugger et al., 2015) and stroke (Westendorp et al., 
2011; Learoyd et al., 2017) are exacerbated by peripheral viral infections. Consequently, our finding that $\mathrm{CXCL} 10$ is the primary protein generated in the brain in response to peripheral viral challenge (Chapters 2 and 3 ) provides a compelling evidence that this chemokine is a putative mediator of the deleterious effects of peripheral viral infections on ongoing neuropathologies.

Another key finding of this dissertation is that neurons are the principal generators of CXCL10. This cellular specificity cannot be overstated. In particular, neurons are highly differentiated cells specialized in electical impuls conduction, and therefore, are not expected to be the main contributors to immune response that is typically attrributed to glial cells, i.e., microglia and astrocytes. Yet, neurons, but not glial cells, generate the overwhelming amounts of CXCL10 in response to PIC challenge. This finding is reminescent of our previous observation of neurons being the primary cells generating the complement factor $B(C f B)$ following PIC challenge (Michalovicz \& Konat, 2015). Consequently, our findings indicate that neurons can carry on important innate immune functions.

Similarly to CXCL10, CXCR3 expression is also restricted to neurons in both naïve and PIC challenged brains (Chapter 3). Neuronal expression of CXCR3 is not surprising, as changes of synaptic function in response to exogenous application of CXCL10 have been demonstrated (Nelson and Gruol, 2004; Bajova et al., 2008; Cho et al., 2009; Kodangattil et al., 2012). Nevertheless, the cellular expression of the chemokine and its cognate receptor demonstrates that the CXCL10/CXCR3 axis operates through autocrine and/or paracrine neuronal networks, where CXCL10 being secreted by a neuron binds to CXCR3 on either the same or a nearby neuron. While neuronal autocrine/paracrine signaling is repeatedly utilized during neurodevelopment (Herrmann and Broihier, 2018), in the adult CNS, this activity is restricted to the synaptic transmission. Also, neurons are not thought as being the main cells to respond to environmental challenges in such a dramatic manner as secreting and reacting to the same factor. Although neurons respond to inflammatory signals, these signals are typically produced by neighboring glial cells (Beattie et al., 2002; Allen, 2014). As such, peripheral PIC-induced CXCL10-CXCR3 signaling provides a unique platform to understand a broader role of neurons beyond the electrical activity.

This study is limited by the use of only female animals, as we are unable to determine if there is a sex difference in PIC-induced antiviral APR and CXCL10/CXCR3 signaling. The decision to use only females stems from the original work by Colm Cunningham examining the induction of 
sickness behavior following peripheral PIC challenge (Jain et al., 2011). The authors made the decision to exclude males from their study due to fighting between male cage mates throughout this and future studies (Fields et al., 1997; Murray et al., 2015) examining peripheral and cerebral inflammatory responses. As such, we continued to add to this literature; however, future studies should utilize both males and females.

\subsection{NEURONAL HYPEREXCITABILITY}

As presented Chapters 3 and 4, CXCR3 mediates the induction of cerebral hyperexcitability following peripheral PIC challenge. The CXCL10-CXCR3 is also responsible for the activation of microglia. Because microglia are potent regulators of synaptic activity, we propose the following mechanism (Fig. 2).

Blood borne cytokines, chemokines, and/or other APR factors, bind to receptors on brain endothelial cells, resulting in the secretion of an unknown factor $(X)$, into the brain parenchyma. Factor $X$ induces neuronal production of CXCL10. Previous studies showing that transfer of plasma from PIC challenged mice into naïve animals induces upregulation of cerebral cytokine and chemokine genes (Fil et al., 2011) strongly supports this contention. Neuronally secreted CXCL10 binds to CXCR3 on the surface of neighboring neurons. The activation of CXCR3 results in the secretion of factor $Y$, that in turn, activates microglia.

Activated microglia impede inhibitory transmission by decoupling GABAergic synapses. Studies from the Trapp lab showing processes of activated microglia invading the synaptic cleft and physically stripping the presynaptic terminals, strongly buttress this mechanism (Chen et al., 2014). However, microglia may hinder inhibitory transmission by secreting soluble factors ( $Z$ ). For example, activated microglia have been shown to secrete bone derived neurotrophic factor (BDNF). BDNF can reverse the chloride anion gradient, resulting in neuronal depolarization following ligation of GABA receptors, turning GABA into an excitatory neurotransmitter (Coull et al., 2005).

Alternatively, microglia may also strengthen excitatory transmission. For example, microglial processes may contact excitatory synapses, strengthen their activity, and thus enhance excitatory transmission (Tremblay et al., 2010; Akiyoshi et al., 2018). 


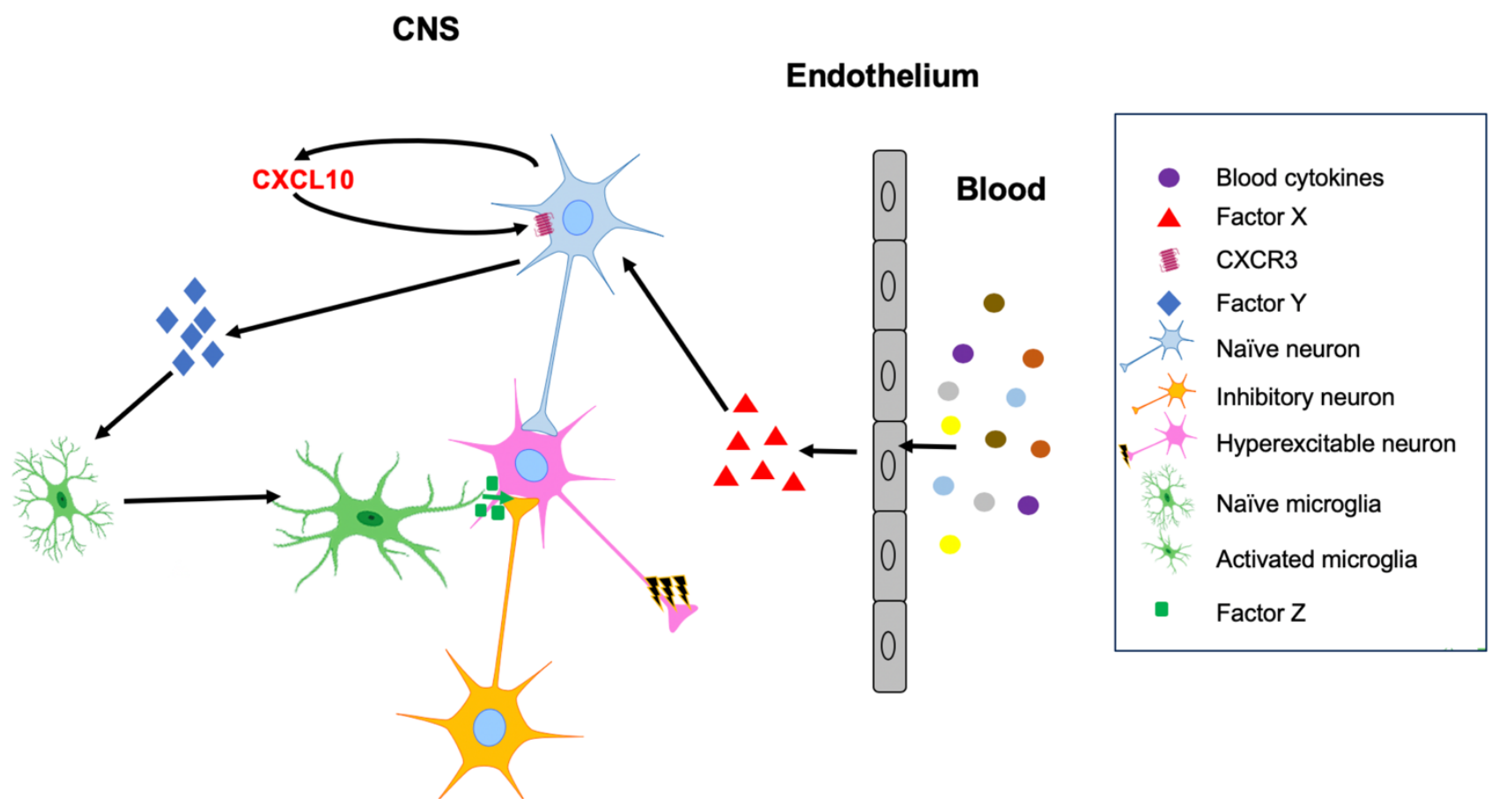

Figure 2. Proposed mechanism of CXCL10/CXCR3-mediated induction of hyperexcitability. Peripheral blood borne cytokines (circles) activate endothelial cells to release an unknown Factor $X$ (triangles) into the CNS parenchyma. Factor $X$ induces neuronal generation and secretion of CXCL10. CXCL10 then acts in an autocrine/paracrine fashion by binding to its receptor, CXCR3, on neurons. CXCR3 activation induces neuronal secretion of Factor Y (diamonds), which in turn, activate microglia. Activated microglia home onto axosomatic synapses of inhibitory neuron (orange) and displace the presynaptic terminals. Alternatively, activated microglia may secrete a soluble Factor $Z$ (squares), disrupting the activity of inhibitory synapses. This de-inhibition renders the pink neuron hyperexcitable. 


\subsection{NEURONAL C-FOS EXPRESSION}

The expression of c-fos has often been utilized as a measure of neuronal activity. For example, seizures, which represent neuronal hyperactivity, profoundly upregulate c-fos expression (BailleLe Crom et al., 1996; Barros et al., 2015). We also observe a dramatic upregulation of neuronal c-fos following PIC challenge. However, PIC challenge does not elicit seizure, and thus does not result in neuronal hyperactivity. Instead, we have shown that PIC challenge renders the neuronal circuits more prone to kainic acid induced seizures (Michalovicz and Konat, 2014; Michalovicz et al., 2015a; Hunsberger et al., 2017). This indicates that the neurons are not firing more but are more likely to fire. This elevated propensity of neurons to fire is defined as hyperexcitability.

Consequently, PIC challenge-increased c-fos expression indicates that c-fos Immunohistochemistry, in addition to assessing neuronal hyperactivity, can also be utilized to examine neuronal hyperexcitability.

Of note, neuronal production of c-fos has been implicated in multiple cellular processes, e.g., the expression of the pro-survival factor, BDNF (Zhang et al., 2002). Ergo, neuronal production of c-fos following PIC challenge may not only serve as an index of neuronal hyperexcitability, but may also provide an insight to the underlying mechanisms.

\subsection{COMORBIDITY OF PIC CHALLENEG IN EAE}

Peripheral viral infections have been demonstrated to be responsible for exacerbations of many neurological disorders (refer to Chapter 1). In particular, infections are a known co-morbidity for MS relapse (Andersen et al., 1993; Panitch, 1994; Edwards et al., 1998; Buljevac et al., 2002; Libbey and Fujinami, 2010). Here, we have verified this comorbidity by showing that PIC challenge has a detrimental effect on mice during the chronic phase of EAE, an animal model of MS (Chapter 5). However, due to a fulminant mortality, we were unable to examine the molecular changes in the brains of these mice. Nevertheless, based on our previous results, CXCL10-CXCR3 signaling is likely a key mediator of this exacerbation. As discussed above, CXCL10 and CXCR3 are both increased in the CSF of MS patients and CXCL10 is expressed by macrophages/microglia and astrocytes in active lesions (Sorensen et al., 2002; Vazirinejad et al., 2014; Iwanowski et al., 2017). As such, further studies are required to verify the role of CXCL10/CXCR3 axis in the EAE model. 


\subsection{CONCLUSIONS}

This study addresses molecular mechanisms by which peripheral PIC challenge induces hyperexcitability of cerebral neurons. We have demonstrated that cerebrally produced CXCL10 chemokine mediates this process. CXCL10 and its cognate receptor CXCR3 are chiefly expressed by neurons, indicating that neuronal autocrine/paracrine loops govern the development of neuronal hyperexcitability. These neuronal loops also activate microglia, potent modulators of synaptic activity. Consequently, we propose that activated microglia impede inhibitory synapses leading to hyperexcitability of neuronal networks.

Furthermore, we have demonstrated that neuronal hyperexcitability can be accurately assessed by quantifying the expression of neuronal c-fos.

In addition, we have developed a preclinical system in which PIC challenge exacerbates the progression of EAE, a murine model of MS. This finding validates detrimental effects of peripheral viral infections in MS patients.

Further elucidation of CXCL10-CXCR3-mediated pathways in future studies will provide a springboard for the development of novel therapeutic approaches to prevent exacerbations of neuropathological diseases.

\subsection{Future Directions}

This dissertation established that the induction of neuronal CXCL10 is a putative mediator of PIC challenge-induced neuronal hyperexcitability. However, the underlying mechanisms have to elucidated. Specifically:

\section{DELINEATE PATHWAY BETWEEN BLOOD BORNE INFLAMMATORY MEDIATORS AND THE NEURONAL GENERATION OF CXCL10}

Previous studies by the Konat lab have shown that transfer of plasma from PIC challenged animals into naïve animals upregulates of cerebral cytokine and chemokine genes (Fil et al., 2011). This finding provides an experimental for the determination of transduction pathways of blood cytokines by endothelial cells. Elucidation of these pathways will provide additional opportunities for therapeutics. 


\section{Delineate neuronal signaling Pathways activated by CXCR3}

While several studies have shown the effect of CXCL10 on neurons, the signaling pathways have not been determined (Nelson and Gruol, 2004; Cho et al., 2009; Kodangattil et al., 2012; Gruol, 2016). The identification of the signaling pathways would provide additional therapeutic targets.

\section{INVOLVEMENT OF MICROGLIA IN THE DEVELOPMENT OF HYPEREXCITABILITY}

The results from Chapter 4.3 indicate that microglia, potent regulators of synaptic activity, are involved in the development of CXCL10-mediated hyperexcitability. We posit that microglia are activated by neuronal factors released in response to CXCL10 ligation. Activated microglia in turn, impede inhibitory synapses, resulting in hyperexcitability. However, mechanisms of the synaptic impairment are not known and also, the enhancement of excitatory synapses is a valid alternative. These mechanisms are very important for therapeutic purposes and therefore should be deciphered in future studies. 


\subsection{REFERENCES}

Akiyoshi R, Wake H, Kato D, Horiuchi H, Ono R, Ikegami A, Haruwaka K, Omori T, Tachibana Y, Moorhouse AJ, Nabekura J (2018) Microglia Enhance Synapse Activity to Promote Local Network Synchronization. eNeuro 5.

Allen NJ (2014) Astrocyte regulation of synaptic behavior. Annu Rev Cell Dev Biol 30:439-463.

Andersen O, Lygner PE, Bergstrom T, Andersson M, Vahlne A (1993) Viral infections trigger multiple sclerosis relapses: a prospective seroepidemiological study. J Neurol 240:417422.

Andrews SP, Cox RJ (2016) Small Molecule CXCR3 Antagonists. J Med Chem 59:2894-2917.

Angiolillo AL, Sgadari C, Taub DD, Liao F, Farber JM, Maheshwari S, Kleinman HK, Reaman $\mathrm{GH}$, Tosato $\mathrm{G}$ (1995) Human interferon-inducible protein 10 is a potent inhibitor of angiogenesis in vivo. J Exp Med 182:155-162.

Baille-Le Crom V, Collombet JM, Burckhart MF, Foquin A, Pernot-Marino I, Rondouin G, Lallement G (1996) Time course and regional expression of C-FOS and HSP70 in hippocampus and piriform cortex following soman-induced seizures. J Neurosci Res 45:513-524.

Bajova H, Nelson TE, Gruol DL (2008) Chronic CXCL10 alters the level of activated ERK1/2 and transcriptional factors CREB and NF-kappaB in hippocampal neuronal cell culture. $J$ Neuroimmunol 195:36-46.

Barros VN, Mundim M, Galindo LT, Bittencourt S, Porcionatto M, Mello LE (2015) The pattern of c-Fos expression and its refractory period in the brain of rats and monkeys. Front Cell Neurosci 9:72.

Beattie EC, Stellwagen D, Morishita W, Bresnahan JC, Ha BK, Von Zastrow M, Beattie MS, Malenka RC (2002) Control of synaptic strength by glial TNFalpha. Science 295:22822285.

Berry KF, M.; Kersey, K.; Stempien, M.; Wagner, F.; van Lier, J.; Sabat, R.; Wolk, K. (2004) Evaluation of T0906487, a CXCR3 antagonist, in a phase 2a psoriasis trial . . In: Inflammation Research Association Biannual meeting, p pS222. Lake George, NY,: Inflammation Research.

Bodnar RJ, Yates CC, Wells A (2006) IP-10 blocks vascular endothelial growth factor-induced endothelial cell motility and tube formation via inhibition of calpain. Circ Res 98:617-625.

Brugger F, Erro R, Balint B, Kagi G, Barone P, Bhatia KP (2015) Why is there motor deterioration in Parkinson's disease during systemic infections-a hypothetical view. NPJ Parkinsons Dis 1:15014.

Buljevac D, Flach HZ, Hop WC, Hijdra D, Laman JD, Savelkoul HF, van Der Meche FG, van Doorn PA, Hintzen RQ (2002) Prospective study on the relationship between infections and multiple sclerosis exacerbations. Brain 125:952-960.

Chen Z, Jalabi W, Hu W, Park HJ, Gale JT, Kidd GJ, Bernatowicz R, Gossman ZC, Chen JT, Dutta R, Trapp BD (2014) Microglial displacement of inhibitory synapses provides neuroprotection in the adult brain. Nat Commun 5:4486.

Cho J, Nelson TE, Bajova H, Gruol DL (2009) Chronic CXCL10 alters neuronal properties in rat hippocampal culture. J Neuroimmunol 207:92-100.

Christensen JE, Nansen A, Moos T, Lu B, Gerard C, Christensen JP, Thomsen AR (2004) Efficient T-cell surveillance of the CNS requires expression of the CXC chemokine receptor 3. J Neurosci 24:4849-4858.

Coull JA, Beggs S, Boudreau D, Boivin D, Tsuda M, Inoue K, Gravel C, Salter MW, De Koninck $Y(2005)$ BDNF from microglia causes the shift in neuronal anion gradient underlying neuropathic pain. Nature 438:1017-1021.

Dabiri S, Kariminik A, Kennedy D (2016) The role of CXCR3 and its ligands in renal transplant outcome. Eur Cytokine Netw 27:34-40. 
Dufour JH, Dziejman M, Liu MT, Leung JH, Lane TE, Luster AD (2002) IFN-gamma-inducible protein 10 (IP-10; CXCL10)-deficient mice reveal a role for IP-10 in effector T cell generation and trafficking. Journal of immunology (Baltimore, Md : 1950) 168:31953204.

Edwards S, Zvartau M, Clarke H, Irving W, Blumhardt LD (1998) Clinical relapses and disease activity on magnetic resonance imaging associated with viral upper respiratory tract infections in multiple sclerosis. J Neurol Neurosurg Psychiatry 64:736-741.

Fields RD, Eshete F, Stevens B, Itoh K (1997) Action potential-dependent regulation of gene expression: temporal specificity in ca2+, cAMP-responsive element binding proteins, and mitogen-activated protein kinase signaling. J Neurosci 17:7252-7266.

Fil D, Borysiewicz E, Konat GW (2011) A broad upregulation of cerebral chemokine genes by peripherally-generated inflammatory mediators. Metab Brain Dis 26:49-59.

Floren LC BK, Tonn G, Ye Q, Wright M, Huang AX, Wang X, Marcus A, John-son M, Collins T et al (2003) 0906487 (T487), a novel CXCR3 antagonist: First time in human study of safety and pharmacokinetics. In: 6th World Congress on Inflammation. Vancouver, Canada: Inflammation Research.

George J, Bleasdale S, Singleton SJ (1997) Causes and prognosis of delirium in elderly patients admitted to a district general hospital. Age Ageing 26:423-427.

Groom JR, Luster AD (2011a) CXCR3 ligands: redundant, collaborative and antagonistic functions. Immunol Cell Biol 89:207-215.

Groom JR, Luster AD (2011b) CXCR3 in T cell function. Exp Cell Res 317:620-631.

Gruol DL (2016) Impact of Increased Astrocyte Expression of IL-6, CCL2 or CXCL10 in Transgenic Mice on Hippocampal Synaptic Function. Brain Sci 6.

Herrmann KA, Broihier HT (2018) What neurons tell themselves: autocrine signals play essential roles in neuronal development and function. Curr Opin Neurobiol 51:70-79.

Holmes C (2013) Review: systemic inflammation and Alzheimer's disease. Neuropathol Appl Neurobiol 39:51-68.

Holmes C, El-Okl M, Williams AL, Cunningham C, Wilcockson D, Perry VH (2003) Systemic infection, interleukin 1 beta, and cognitive decline in Alzheimer's disease. J Neurol Neurosurg Psychiatry 74:788-789.

Hueso L, Ortega R, Selles F, Wu-Xiong NY, Ortega J, Civera M, Ascaso JF, Sanz MJ, Real JT, Piqueras L (2018) Upregulation of angiostatic chemokines IP-10/CXCL10 and ITAC/CXCL11 in human obesity and their implication for adipose tissue angiogenesis. Int J Obes (Lond) 42:1406-1417.

Hunsberger HC, Konat GW, Reed MN (2017) Peripheral viral challenge elevates extracellular glutamate in the hippocampus leading to seizure hypersusceptibility. J Neurochem 141:341-346.

Iwanowski P, Losy J, Kramer L, Wojcicka M, Kaufman E (2017) CXCL10 and CXCL13 chemokines in patients with relapsing remitting and primary progressive multiple sclerosis. J Neurol Sci 380:22-26.

Jain S, Gautam V, Naseem S (2011) Acute-phase proteins: As diagnostic tool. J Pharm Bioallied Sci 3:118-127.

Kariya T, Ueta H, Xu XD, Koga D, Ezaki T, Yu E, Kusumi S, Kitazawa Y, Sawanobori Y, Ushiki T, Issekutz T, Matsuno K (2016) Direct evidence for activated CD8+ T cell transmigration across portal vein endothelial cells in liver graft rejection. J Gastroenterol 51:985-998.

Klein RS (2004) Regulation of neuroinflammation: the role of CXCL10 in lymphocyte infiltration during autoimmune encephalomyelitis. J Cell Biochem 92:213-222.

Klein RS, Lin E, Zhang B, Luster AD, Tollett J, Samuel MA, Engle M, Diamond MS (2005) Neuronal CXCL10 directs CD8+ T-cell recruitment and control of West Nile virus encephalitis. J Virol 79:11457-11466. 
Kodangattil JN, Gorji A, Moddel G, Muller M, Weber W (2012) The inflammatory chemokine CXCL10 modulates synaptic plasticity and neuronal activity in the hippocampus.

European Journal of Inflammation 10:311-328.

Krauthausen M, Kummer MP, Zimmermann J, Reyes-Irisarri E, Terwel D, Bulic B, Heneka MT, Muller M (2015) CXCR3 promotes plaque formation and behavioral deficits in an Alzheimer's disease model. The Journal of clinical investigation 125:365-378.

Learoyd AE et al. (2017) Infections Up to 76 Days After Stroke Increase Disability and Death. Transl Stroke Res 8:541-548.

Lee JH, Kim B, Jin WJ, Kim HH, Ha H, Lee ZH (2017) Pathogenic roles of CXCL10 signaling through CXCR3 and TLR4 in macrophages and T cells: relevance for arthritis. Arthritis Res Ther 19:163.

Libbey JE, Fujinami RS (2010) Potential triggers of MS. Results Probl Cell Differ 51:21-42.

Mayer L, Sandborn WJ, Stepanov Y, Geboes K, Hardi R, Yellin M, Tao X, Xu LA, Salter-Cid L, Gujrathi S, Aranda R, Luo AY (2014) Anti-IP-10 antibody (BMS-936557) for ulcerative colitis: a phase II randomised study. Gut 63:442-450.

Michalovicz LT, Konat GW (2014) Peripherally restricted acute phase response to a viral mimic alters hippocampal gene expression. Metab Brain Dis 29:75-86.

Michalovicz LT, Lally B, Konat GW (2015) Peripheral challenge with a viral mimic upregulates expression of the complement genes in the hippocampus. J Neuroimmunol 285:137142.

Michlmayr D, McKimmie CS (2014a) Role of CXCL10 in central nervous system inflammation. Int $\mathrm{J}$ Interferon Cytokine Mediators REs 6:1-18.

Michlmayr D, McKimmie CS (2014b) Role of CXCL10 in central nervous system inflammation. Int J Interferon Cytokine Mediators Res 6:1-18.

Mills Ko E, Ma JH, Guo F, Miers L, Lee E, Bannerman P, Burns T, Ko D, Sohn J, Soulika AM, Pleasure D (2014) Deletion of astroglial CXCL10 delays clinical onset but does not affect progressive axon loss in a murine autoimmune multiple sclerosis model. $\mathrm{J}$ Neuroinflammation 11:105.

Müller M, Carter SL, Hofer MJ, Manders P, Getts DR, Getts MT, Dreykluft A, Lu B, Gerard C, King NJC, Campbell IL (2007) CXCR3 Signaling Reduces the Severity of Experimental Autoimmune Encephalomyelitis by Controlling the Parenchymal Distribution of Effector and Regulatory T Cells in the Central Nervous System. The Journal of Immunology 179:2774-2786.

Murray AM, Levkoff SE, Wetle TT, Beckett L, Cleary PD, Schor JD, Lipsitz LA, Rowe JW, Evans DA (1993) Acute delirium and functional decline in the hospitalized elderly patient. J Gerontol 48:M181-186.

Murray C, Griffin EW, O'Loughlin E, Lyons A, Sherwin E, Ahmed S, Stevenson NJ, Harkin A, Cunningham $C$ (2015) Interdependent and independent roles of type I interferons and IL6 in innate immune, neuroinflammatory and sickness behaviour responses to systemic poly I:C. Brain Behav Immun 48:274-286.

Nee LE, Lippa CF (1999) Alzheimer's disease in 22 twin pairs--13-year follow-up: hormonal, infectious and traumatic factors. Dement Geriatr Cogn Disord 10:148-151.

Nelson TE, Gruol DL (2004) The chemokine CXCL10 modulates excitatory activity and intracellular calcium signaling in cultured hippocampal neurons. J Neuroimmunol 156:7487.

Panitch HS (1994) Influence of infection on exacerbations of multiple sclerosis. Ann Neurol 36 Suppl:S25-28.

Roberts WK, Blachere NE, Frank MO, Dousmanis A, Ransohoff RM, Darnell RB (2015) A destructive feedback loop mediated by CXCL10 in central nervous system inflammatory disease. Ann Neurol 78:619-629. 
Sorensen TL, Trebst C, Kivisakk P, Klaege KL, Majmudar A, Ravid R, Lassmann H, Olsen DB, Strieter RM, Ransohoff RM, Sellebjerg F (2002) Multiple sclerosis: a study of CXCL10 and CXCR3 co-localization in the inflamed central nervous system. J Neuroimmunol 127:59-68.

Tellez-Zenteno JF, Matijevic S, Wiebe S (2005) Somatic comorbidity of epilepsy in the general population in Canada. Epilepsia 46:1955-1962.

Tremblay ME, Lowery RL, Majewska AK (2010) Microglial interactions with synapses are modulated by visual experience. PLoS Biol 8:e1000527.

Vazirinejad R, Ahmadi Z, Kazemi Arababadi M, Hassanshahi G, Kennedy D (2014) The biological functions, structure and sources of CXCL10 and its outstanding part in the pathophysiology of multiple sclerosis. Neuroimmunomodulation 21:322-330.

Verrotti A, Tocco AM, Coppola GG, Altobelli E, Chiarelli F (2009) Afebrile benign convulsions with mild gastroenteritis: a new entity? Acta Neurol Scand 120:73-79.

Vezzani A, Granata T (2005) Brain inflammation in epilepsy: experimental and clinical evidence. Epilepsia 46:1724-1743.

Westendorp WF, Nederkoorn PJ, Vermeij JD, Dijkgraaf MG, van de Beek D (2011) Post-stroke infection: a systematic review and meta-analysis. BMC Neurol 11:110.

Wijtmans M, VerzijI D, Leurs R, de Esch IJ, Smit MJ (2008) Towards small-molecule CXCR3 ligands with clinical potential. ChemMedChem 3:861-872.

Yellin M, Paliienko I, Balanescu A, Ter-Vartanian S, Tseluyko V, Xu LA, Tao X, Cardarelli PM, Leblanc H, Nichol G, Ancuta C, Chirieac R, Luo A (2012) A phase II, randomized, double-blind, placebo-controlled study evaluating the efficacy and safety of MDX-1100, a fully human anti-CXCL10 monoclonal antibody, in combination with methotrexate in patients with rheumatoid arthritis. Arthritis Rheum 64:1730-1739.

Zhang J, Zhang D, McQuade JS, Behbehani M, Tsien JZ, Xu M (2002) c-fos regulates neuronal excitability and survival. Nat Genet 30:416-420.

Zheng KS, Dorfman BJ, Christos PJ, Khadem NR, Henchcliffe C, Piboolnurak P, Nirenberg MJ (2012) Clinical characteristics of exacerbations in Parkinson disease. Neurologist 18:120-124. 